\title{
Sum rules in the heavy quark limit of QCD
}

\author{
A. Le Yaouanc, L. Oliver and J.-C. Raynal \\ Laboratoire de Physique Théorique ${ }^{1}$ \\ Université de Paris XI, Bâtiment 210, 91405 Orsay Cedex, France
}

\begin{abstract}
In the leading order of the heavy quark expansion, we propose a method within the OPE and the trace formalism, that allows to obtain, in a systematic way, Bjorken-like sum rules for the derivatives of the elastic Isgur-Wise function $\xi(w)$ in terms of corresponding Isgur-Wise functions of transitions to excited states. A key element is the consideration of the non-forward amplitude, as introduced by Uraltsev. A simplifying feature of our method is to consider currents aligned along the initial and final four-velocities. As an illustration, we give a very simple derivation of Bjorken and Uraltsev sum rules. On the other hand, we obtain a new class of sum rules that involve the products of IW functions at zero recoil and IW functions at any $w$. Special care is given to the needed derivation of the projector on the polarization tensors of particles of arbitrary integer spin. The new sum rules give further information on the slope $\rho^{2}=-\xi^{\prime}(1)$ and also on the curvature $\sigma^{2}=\xi^{\prime \prime}(1)$, and imply, modulo a very natural assumption, the inequality $\sigma^{2} \geq \frac{5}{4} \rho^{2}$, and therefore the absolute bound $\sigma^{2} \geq \frac{15}{16}$.
\end{abstract}

LPT Orsay 02-29

July 2002

e-mails : leyaouan@th.u-psud.fr, oliver@th.u-psud.fr

\footnotetext{
${ }^{1}$ Unité Mixte de Recherche UMR 8627 - CNRS
} 


\section{Introduction.}

Since the formulation of Bjorken sum rule [1], other sum rules (SR) have been derived involving leading and subleading quantities in the heavy quark expansion $[2,3,4,5,6]$. The recent Uraltsev SR [6, 7] at leading order came as a big surprise, leading to the rigorous lower bound for the elastic Isgur-Wise function $\rho^{2} \geq 3 / 4^{2}$. As with earlier SR, one gets the impression that these results come out like a fishing in a lake, swarming with sum rules, the success of the catch depending on the genius or skill of the particular authors. Hence the necessity of having a systematic way of formulating these SR. This is the subject of the present paper, although only in the particular case of IW functions in the heavy quark limit of QCD. The method can be easily applied to subleading form factors [8].

In the derivation of the sum rules we will make use of the Operator Product Expansion (OPE) [9] in heavy quark transitions $[2,5,6,10]$, in a manifestly covariant approach.

To be completely general, let us consider the direct graphs $B_{i}\left(v_{i}\right) \stackrel{\Gamma_{1}}{\rightarrow} D^{(n)}\left(v^{\prime}\right) \stackrel{\Gamma_{2}}{\rightarrow} B_{f}\left(v_{f}\right)$, where $B_{i}$ and $B_{f}$ are ground state $B$ or $B^{*}$ mesons and $D^{(n)}$ are all possible ground state or excited $D$ mesons coupled to $B_{i}$ and $B_{f}$ through the currents $\bar{h}_{c}\left(v^{\prime}\right) \Gamma_{1} h_{b}\left(v_{i}\right)$ and $\bar{h}_{b}\left(v_{f}\right) \Gamma_{2} h_{c}\left(v^{\prime}\right)$. The Dirac matrices $\Gamma_{i}(i=1,2)$ are arbitrary and can be chosen to derive relations involving definite current matrix elements.

Let us summarize the general argument. We consider two arbitrary currents :

$$
J_{1}(x)=\bar{c}(x) \Gamma_{1} b(x) \quad J_{2}(y)=\bar{b}(y) \Gamma_{2} c(y)
$$

and their $T$ product

$$
T_{f i}(q)=i \int d^{4} x e^{-i q \cdot x}<B_{f}\left|T\left[J_{2}(x) J_{1}(0)\right]\right| B_{i}>
$$

As explained in detail for example in ref. [5], inserting in this expression intermediate states, $x<0$ receives contributions from the direct channel with a single heavy quark $c$, while $x>0$ receives contributions from intermediate states with $b \bar{c} b$ quarks, the $Z$ diagrams. The energy denominators are $M_{B}-q^{0}-E_{X_{c}}$ for the direct graphs and $M_{B}+q^{0}-\left(E_{X_{c}^{\prime}}+2 M_{B}\right)$ for the $Z$ diagrams. Taking the typical

\footnotetext{
${ }^{2}$ This bound was obtained in a class of relativistic quark models ([11], [12]), that were afterwards shown to satisfy Uraltsev sum rule [13].
} 
virtuality of the direct channels $V=M_{B}-q^{0}-E_{X_{c}}$ such that $\Lambda_{Q C D} \ll V \ll M_{B}$, one sees that the direct channels contribute at the order $1 / V$ and the $Z$ diagrams at the order $1 /\left(-V-2 M_{D}\right)$. In both cases the absolute value of the denominator is $\gg \Lambda_{Q C D}$. This allows to approximate (2) with the leading contribution to the OPE [10] :

$$
T_{f i}(q)=i \int d^{4} x e^{-i q \cdot x}<B_{f}\left|\bar{b}(x) \Gamma_{2} S_{c}(x, 0) \Gamma_{1} b(0)\right| B_{i}>+O\left(1 / m_{c}^{2}\right)
$$

where $S_{c}(x, 0)$ is the free charm quark propagator if $O\left(\alpha_{s}\right)$ corrections are neglected. The $c$ quark propagator has two terms, a positive energy denominator $\sim V$ and a negative energy denominator $\sim\left(-V-2 m_{c}\right)$. Varying $V$ independently of $m_{c}$ one can equate the direct channel contribution to (2) to the one of the positive energy pole of the $c$ quark propagator in (3), the so-called OPE side, giving the following result that involves only the direct channel :

$$
\begin{aligned}
& \frac{1}{2 v^{\prime 0} \sqrt{4 v_{i}^{0} v_{f}^{0}}} \\
& \left\{\sum_{D=P, V} \sum_{n} \operatorname{Tr}\left[\overline{\mathcal{B}}_{f}\left(v_{f}\right) \bar{\Gamma}_{2} \mathcal{D}^{(n)}\left(v^{\prime}\right)\right] \operatorname{Tr}\left[\overline{\mathcal{D}}^{(n)}\left(v^{\prime}\right) \Gamma_{1} \mathcal{B}_{i}\left(v_{i}\right)\right] \xi^{(n)}\left(w_{i}\right) \xi^{(n) *}\left(w_{f}\right)\right. \\
& \left.+ \text { Contribution from other excited states }+O\left(1 / m_{Q}\right)\right\} \\
& =-\frac{1}{\sqrt{4 v_{i}^{0} v_{f}^{0}}} \xi\left(w_{i f}\right) \operatorname{Tr}\left[\overline{\mathcal{B}}_{f}\left(v_{f}\right) \Gamma_{2} \frac{\psi^{\prime}{ }_{c}+1}{2 v_{c}^{\prime}} \Gamma_{1} \mathcal{B}_{i}\left(v_{i}\right)\right]+O\left(1 / m_{Q}\right) .
\end{aligned}
$$

In this equation, $\frac{\underline{\prime}_{c}^{\prime}+1}{2 v_{c}^{\prime 0}}$ is the positive energy residue of the $c$ quark propagator and the 1.h.s. is the sum over all possible ground state or excited $D$ mesons. We have adopted the trace formalism for the current matrix elements [4, 14] and made explicit in (4) the sum over pseudoscalar and vector $D\left(D^{*}\right)$ mesons and their radial quantum number. In relation (4)

$$
w_{i}=v_{i} \cdot v^{\prime} \quad, \quad w_{f}=v_{f} \cdot v^{\prime} \quad, \quad w_{i f}=v_{i} \cdot v_{f}
$$

In the 1.h.s. there are also leading order contributions of excited states and subleading terms coming from the ground state or from transitions between the ground state and excited states, denoted by $O\left(1 / m_{Q}\right)$, where $m_{Q}$ can be $m_{c}$ or $m_{b}$.

One main point we want to emphasize is that in the OPE side the ground state IW function $\xi\left(w_{i f}\right)$ appears since, following Uraltsev [6] we assume in general $v_{i} \neq v_{f}$ 
and take $B_{i}$ and $B_{f}$ to be ground state $B$ mesons. Of course, for $w_{i f}=1$ one gets $\xi(1)=1, w_{i}=w_{f}=w$ and the general formula (4) takes the more familiar form $[5]:$

$$
\begin{aligned}
& \frac{1}{4 v^{0} v^{\prime 0}}\left\{\sum_{D=P, V} \sum_{n} \operatorname{Tr}\left[\overline{\mathcal{B}}_{f}(v) \bar{\Gamma}_{2} \mathcal{D}^{(n)}\left(v^{\prime}\right)\right] \operatorname{Tr}\left[\overline{\mathcal{D}}^{(n)}\left(v^{\prime}\right) \Gamma_{1} \mathcal{B}_{i}(v)\right]\left|\xi^{(n)}(w)\right|^{2}\right. \\
& \left.+ \text { Contribution from other excited states }+O\left(1 / m_{Q}\right)\right\} \\
& =-\frac{1}{2 v^{0}} \operatorname{Tr}\left[\overline{\mathcal{B}}_{f}(v) \Gamma_{2} \frac{\psi_{c}^{\prime}+1}{2 v_{c}^{\prime 0}} \Gamma_{1} \mathcal{B}_{i}(v)\right]+O\left(1 / m_{Q}^{2}\right) .
\end{aligned}
$$

But let us keep to the general case $v_{i} \neq v_{f}$. By choosing in a convenient way the initial and final mesons $B_{i}$ and $B_{f}$ and the Dirac matrices $\Gamma_{1}$ and $\Gamma_{2}$, one can derive sum rules at the leading order (Bjorken SR [1] and Uraltsev SR [6]) and also SR involving subleading Isgur-Wise functions, as we have obtained in ref. [5]. To illustrate the method, we will limit ourselves in this paper to the heavy quark limit.

In the heavy quark limit, since we can make the four-velocity of the intermediate quark equal to the intermediate hadron velocity, $v_{c}^{\prime}=v^{\prime}$, relation (4) writes, multiplying by $2 v_{0}^{\prime} \sqrt{4 v_{i}^{0} v_{f}^{0}}$

$$
L\left(w_{i f}, w_{i}, w_{f}\right)=R\left(w_{i f}, w_{i}, w_{f}\right)
$$

where $L\left(w_{i f}, w_{i}, w_{f}\right)$ stands for the l.h.s. (the sum over intermediate states $\mathcal{D}^{(n)}\left(v^{\prime}\right)$ ) and $R\left(w_{i f}, w_{i}, w_{f}\right)$ stands for the r.h.s. (the OPE side, proportional to $\xi\left(w_{i f}\right)$ ).

The variables $w_{i f}, w_{i}$ and $w_{f}$ are independent within a certain domain. Indeed, without loss of generality one can take

$$
v_{i}=(1,0,0,0) \quad v_{f}=\left(\sqrt{1+a^{2}}, 0,0, a\right) \quad v^{\prime}=\left(\sqrt{1+b^{2}+c^{2}}, 0, b, c\right)
$$

giving

$$
w_{i f}=\sqrt{1+a^{2}} \quad w_{i}=\sqrt{1+b^{2}+c^{2}} \quad w_{f}=\sqrt{1+b^{2}+c^{2}} \sqrt{1+a^{2}}-a c .
$$

One has three independent parameters $a, b$ and $c$ or equivalently $w_{i}, w_{f}$ and $w_{i f}$ that lie within a limited domain. The domain of $\left(w_{i f}, w_{i}, w_{f}\right)$ is

$$
w_{i f} \geq 1 \quad, \quad 2 w_{i f} w_{i} w_{f}-w_{i f}^{2}-w_{i}^{2}-w_{f}^{2}+1 \geq 0
$$


that implies

$$
w_{i} \geq 1 \quad, \quad w_{f} \geq 1
$$

and is equivalent to

$$
\begin{gathered}
w_{i} \geq 1 \quad, \quad w_{f} \geq 1 \\
w_{i} w_{f}-\sqrt{\left(w_{i}^{2}-1\right)\left(w_{f}^{2}-1\right)} \leq w_{i f} \leq w_{i} w_{f}+\sqrt{\left(w_{i}^{2}-1\right)\left(w_{f}^{2}-1\right)} .
\end{gathered}
$$

There is a subdomain for $w_{i}=w_{f}=w$, namely :

$$
w \geq 1 \quad, \quad 1 \leq w_{i f} \leq 2 w^{2}-1
$$

Within this domain one can differentiate relatively to any of these variables,

$$
\frac{\partial^{p+q+r} L}{\partial w_{i f}^{p} \partial w_{i}^{q} \partial w_{f}^{r}}=\frac{\partial^{p+q+r} R}{\partial w_{i f}^{p} \partial w_{i}^{q} \partial w_{f}^{r}}
$$

and obtain different sum rules taking different limits to the frontier of the domain, e.g.,

$$
\begin{array}{ll} 
& w_{i f} \rightarrow 1, w_{i}=w_{f}=w \\
\text { or } & w_{i} \rightarrow 1, w_{i f}=w_{f}=w \\
\text { or } & w_{f} \rightarrow 1, w_{i f}=w_{i}=w .
\end{array}
$$

A last general remark. In the SR we will consider the sum over discrete intermediate ground state or excited D mesons. However, our results have a wider generality, as they can include a possible continuum. Such a continuum would only be a slight technical complication, as it can also be expanded into $j^{P}$ states, and the sum over discrete states would become an integral, without any conceptual change in the final results.

The paper is organized as follows. In Section 2 we write down the general form of the SR in the heavy quark limit for a general pair of currents $\bar{h}_{v^{\prime}}^{(c)} \Gamma_{1} h_{v_{i}}^{(b)}, \bar{h}_{v_{f}}^{(b)} \Gamma_{2} h_{v^{\prime}}^{(c)}$, making explicit the intermediate states $\frac{1}{2}^{-}, \frac{1}{2}^{+}, \frac{3}{2}^{+}$, and $\frac{3}{2}^{-}$as well, in order to have a control on high powers of the recoil $(w-1)$. In Section 3 we derive the sum rules (in particular Bjorken and Uraltsev SR) for the axial currents $\left\{\Gamma_{1}, \Gamma_{2}\right\}=\left\{\psi_{i} \gamma_{5}, \psi_{f} \gamma_{5}\right\}$ and in Section 4 similarly for the vector currents $\left\{\Gamma_{1}, \Gamma_{2}\right\}=\left\{\psi_{i}, \psi_{f}\right\}$. In Section 5 
we underline a new class of sum rules with implications, in particular, for the slope and curvature of $\xi(w)$. Moreover, we demonstrate that higher excited states give a vanishing contribution to these SR. In Section 6 we write down a lower bound on the curvature of $\xi(w)$ and in Section 7 we point out some phenomenological remarks in connection with the Bakamjian-Thomas class of relativistic quark models. In Section 8 we conclude. In Appendix A we construct the general formula for the projector on the polarization tensors of particles of arbitrary spin. With it, we deduce a formula that is needed in the calculation of the contributions to the sum rules of higher excited states. Using this general result, we have recently obtained rigorous bounds on all derivatives of the IW function $\xi(w)$ [15]. For the curvature $\sigma^{2}=\xi^{\prime \prime}(1)$ we find in the present paper the same bound using a different method and making a sensible phenomenological hypothesis. Finally, in Appendix B we give a derivation of Bjorken and Uraltsev SR with the currents $\left\{\Gamma_{1}, \Gamma_{2}\right\}=\left\{\psi_{i}, \psi_{i}\right\}$ and initial and final states $B^{*\left(\lambda_{i}\right)}\left(v_{i}\right), B^{*\left(\lambda_{f}\right)}\left(v_{f}\right)$, a manifestly covariant version of those states and currents used by Uraltsev, $\left\{\Gamma_{1}, \Gamma_{2}\right\}=\left\{\gamma^{0}, \gamma^{0}\right\}$ in the rest frame of the initial $B^{*\left(\lambda_{i}\right)}(1, \mathbf{0})$. Of course, this choice of the vector current would make simpler the calculation of radiative corrections to the sum rules than in the case, say, of the axial current. But radiative corrections are outside the scope of the present paper, that adopts the strict heavy quark limit.

\section{General form of the sum rules in the heavy quark limit.}

The r.h.s. writes, in the heavy quark limit, since then $v_{c}^{\prime}=v^{\prime}$ :

$$
R\left(w_{i f}, w_{i}, w_{f}\right)=-2 \xi\left(w_{i f}\right) \operatorname{Tr}\left[\overline{\mathcal{B}}_{f}\left(v_{f}\right) \Gamma_{2} P_{+}^{\prime} \Gamma_{1} \mathcal{B}_{i}\left(v_{i}\right)\right]
$$

Let us decompose the l.h.s. into contributions of the different intermediate states : as intermediate states, we will consider the $0_{1 / 2}^{-}, 1_{1 / 2}^{-}$, and the orbitally excited states $2_{3 / 2}^{+}, 1_{3 / 2}^{+}, 0_{1 / 2}^{+}, 1_{1 / 2}^{+}$(with the tower of their radial excitations). Moreover, to have some control of the SR near zero recoil, it is important to have an idea of the contributions of higher orbital excitations. To this purpose, we will take into account the $\frac{3}{2}^{-}$intermediate states, namely the states $2_{3 / 2}^{-}$and $1_{3 / 2}^{-}$. 
Let us now write down the $4 \times 4$ matrices of the lower $j^{P}$ states $[4,14]$. The matrices for the $\frac{1}{2}^{-}$mesons read :

$$
\begin{aligned}
0_{1 / 2}^{-}: & \mathcal{M}(v)=P_{+}\left(-\gamma_{5}\right) \\
1_{1 / 2}^{-}: & \mathcal{M}(v)=P_{+} \varepsilon_{v}^{\mu} \gamma_{\mu}
\end{aligned}
$$

where $P_{+}$is the projector :

$$
P_{+}=\frac{1+\not}{2}
$$

The $4 \times 4$ matrices of the $\frac{3}{2}^{+}$states are given by the four-vectors :

$$
\begin{aligned}
2_{3 / 2}^{+}: & \mathcal{M}^{\mu}(v)=P_{+} \varepsilon_{v}^{\mu \nu} \gamma_{\mu} \\
1_{3 / 2}^{+}: & \mathcal{M}^{\mu}(v)=-\sqrt{\frac{3}{2}} P_{+} \varepsilon_{v}^{\nu} \gamma_{5}\left[g_{\nu}^{\mu}-\frac{1}{3} \gamma_{\nu}\left(\gamma^{\mu}-v^{\mu}\right)\right]
\end{aligned}
$$

and those of the $\frac{1}{2}^{+}$states are given by [4] :

$$
\begin{array}{ll}
0_{1 / 2}^{+}: & \mathcal{M}(v)=P_{+} \\
1_{1 / 2}^{+}: & \mathcal{M}(v)=P_{+} \varepsilon_{v}^{\mu} \gamma_{5} \gamma_{\mu} .
\end{array}
$$

Finally, those of the $\frac{3}{2}^{-}$states will be obtained from (19) by multiplying on the right by $\left(-\gamma_{5}\right)$ :

$$
\begin{aligned}
2_{3 / 2}^{-}: & \mathcal{M}^{\mu}(v)=P_{+} \varepsilon_{v}^{\mu \nu} \gamma_{\nu}\left(-\gamma_{5}\right) \\
1_{3 / 2}^{-}: & \mathcal{M}^{\mu}(v)=\sqrt{\frac{3}{2}} P_{+} \varepsilon_{v}^{\nu}\left[g_{\nu}^{\mu}-\frac{1}{3} \gamma_{\nu}\left(\gamma^{\mu}+v^{\mu}\right)\right] .
\end{aligned}
$$

The corresponding matrix elements, for a current given by the Dirac matrix $\Gamma$, $\operatorname{read}[4]$ :

$$
\begin{aligned}
& <D^{(n)}\left(\frac{1}{2}^{-}\right)\left(v^{\prime}\right)\left|\bar{h}_{v^{\prime}}^{(c)} \Gamma h_{v}^{(b)}\right| B\left(\frac{1}{2}^{-}\right)(v)>=\xi^{(n)}(w) \operatorname{Tr}\left[\overline{\mathcal{D}}\left(v^{\prime}\right) \Gamma \mathcal{B}(v)\right]
\end{aligned}
$$

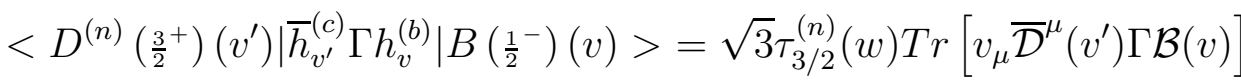

$$
\begin{aligned}
& <D^{(n)}\left(\frac{1}{2}^{+}\right)\left(v^{\prime}\right)\left|\bar{h}_{v^{\prime}}^{(c)} \Gamma h_{v}^{(b)}\right| B\left(\frac{1}{2}^{-}\right)(v)>=2 \tau_{1 / 2}^{(n)}(w) \operatorname{Tr}\left[\overline{\mathcal{D}}\left(v^{\prime}\right) \Gamma \mathcal{B}(v)\right] \\
& \left.<D^{(n)}{\left(\frac{3}{2}^{-}\right.}^{-}\right)\left(v^{\prime}\right)\left|\bar{h}_{v^{\prime}}^{(c)} \Gamma h_{v}^{(b)}\right| B\left(\frac{1}{2}^{-}\right)(v)>=\sqrt{3} \sigma_{3 / 2}^{(n)}(w) \operatorname{Tr}\left[v_{\mu} \overline{\mathcal{D}}^{\mu}\left(v^{\prime}\right) \Gamma \mathcal{B}(v)\right]
\end{aligned}
$$

where $w=v \cdot v^{\prime}, n$ is a radial quantum number and, in analogy with $\tau_{3 / 2}(w)$, we have called $\sigma_{3 / 2}(w)$ the IW function between the ground state and the $\frac{3}{2}^{-}$states. 
As pointed out in [4], $\sigma_{3 / 2}(w)$ need not to vanish at $w=1$, since the current matrix elements vanish in the heavy quark limit. The notation $\xi^{(n)}(w), \tau_{1 / 2}^{(n)}(w)$ and $\tau_{3 / 2}^{(n)}(w)$ is the one of Isgur and Wise [1].

In what follows, we set the different IW functions to be real.

The contributions of the $0_{1 / 2}^{-}, 1_{1 / 2}^{-}$states write :

$$
\begin{gathered}
L\left(0_{1 / 2}^{-}\right)=\operatorname{Tr}\left[\overline{\mathcal{B}}_{f}\left(v_{f}\right) \bar{\Gamma}_{2} P_{+}^{\prime}\left(-\gamma_{5}\right)\right] \operatorname{Tr}\left[\left(-\gamma_{5}\right) P_{+}^{\prime} \Gamma_{1} \mathcal{B}_{i}\left(v_{i}\right)\right] \sum_{n} \xi^{(n)}\left(w_{i}\right) \xi^{(n)}\left(w_{f}\right) \\
L\left(1_{1 / 2}^{-}\right)=\sum_{\lambda} \varepsilon^{\prime(\lambda) \mu} \varepsilon^{\prime(\lambda) \nu} \operatorname{Tr}\left[\overline{\mathcal{B}}_{f}\left(v_{f}\right) \bar{\Gamma}_{2} P_{+}^{\prime} \gamma_{\nu}\right] \operatorname{Tr}\left[\gamma_{\mu} P_{+}^{\prime} \Gamma_{1} \mathcal{B}_{i}\left(v_{i}\right)\right] \sum_{n} \xi^{(n)}\left(w_{i}\right) \xi^{(n)}\left(w_{f}\right) .
\end{gathered}
$$

The contribution of the parity + excited states $2_{3 / 2}^{+}, 1_{3 / 2}^{+}, 0_{1 / 2}^{+}, 1_{1 / 2}^{+}$is given by the following expressions :

$$
\begin{gathered}
L\left(2_{3 / 2}^{+}\right)=\sum_{\lambda} \varepsilon^{\prime(\lambda) \mu \nu} \varepsilon^{\prime(\lambda) * \rho \sigma} \operatorname{Tr}\left[v_{f \rho} \overline{\mathcal{B}}_{f} \bar{\Gamma}_{2} P_{+}^{\prime} \gamma_{\sigma}\right] \operatorname{Tr}\left[v_{i \mu} \gamma_{\nu} P_{+}^{\prime} \Gamma_{1} \mathcal{B}_{i}\right] 3 \sum_{n} \tau_{3 / 2}^{(n)}\left(w_{i}\right) \tau_{3 / 2}^{(n)}\left(w_{f}\right) \\
L\left(1_{3 / 2}^{+}\right)=\sum_{\lambda} \varepsilon^{\prime(\lambda) \nu} \varepsilon^{\prime(\lambda) * \sigma} \frac{3}{2} \operatorname{Tr}\left\{\overline{\mathcal{B}}_{f} \bar{\Gamma}_{2} P_{+}^{\prime} \gamma_{5}\left[v_{f \sigma}-\frac{1}{3} \gamma_{\sigma}\left(\psi_{f}-w_{f}\right)\right]\right\} \\
\operatorname{Tr}\left\{\left[v_{i \nu}-\frac{1}{3}\left(\psi_{i}-w_{i}\right) \gamma_{\nu}\right]\left(-\gamma_{5}\right) P_{+}^{\prime} \Gamma_{1} \mathcal{B}_{i}\right\} 3 \sum_{n} \tau_{3 / 2}^{(n)}\left(w_{i}\right) \tau_{3 / 2}^{(n)}\left(w_{f}\right) \\
L\left(0_{1 / 2}^{+}\right)=\operatorname{Tr}\left[\overline{\mathcal{B}}_{f} \bar{\Gamma}_{2} P_{+}^{\prime}\right] \operatorname{Tr}\left[P_{+}^{\prime} \Gamma_{1} \mathcal{B}_{i}\right] 4 \sum_{n} \tau_{1 / 2}^{(n)}\left(w_{i}\right) \tau_{1 / 2}^{(n)}\left(w_{f}\right) \\
L\left(1_{1 / 2}^{+}\right)=\sum_{\lambda} \varepsilon^{\prime(\lambda) \mu} \varepsilon^{\prime(\lambda) * \nu} \operatorname{Tr}\left[\overline{\mathcal{B}}_{f} \bar{\Gamma}_{2} P_{+}^{\prime} \gamma_{5} \gamma_{\mu}\right] \operatorname{Tr}\left[\gamma_{\nu}\left(-\gamma_{5}\right) P_{+}^{\prime} \Gamma_{1} \mathcal{B}_{i}\right] 4 \sum_{n} \tau_{1 / 2}^{(n)}\left(w_{i}\right) \tau_{1 / 2}^{(n)}\left(w_{f}\right)
\end{gathered}
$$

$$
\begin{aligned}
& L\left(2_{3 / 2}^{-}\right)=\sum_{\lambda} \varepsilon^{\prime(\lambda) \mu \nu} \varepsilon^{\prime(\lambda) * \rho \sigma} \operatorname{Tr}\left[v_{f \rho} \overline{\mathcal{B}}_{f} \bar{\Gamma}_{2} P_{+}^{\prime} \gamma_{\sigma}\left(-\gamma_{5}\right)\right] \\
& \operatorname{Tr}\left[v_{i \mu} \gamma_{5} \gamma_{\nu} P_{+}^{\prime} \Gamma_{1} \mathcal{B}_{i}\right] 3 \sum_{n} \sigma_{3 / 2}^{(n)}\left(w_{i}\right) \sigma_{3 / 2}^{(n)}\left(w_{f}\right)
\end{aligned}
$$

$$
\begin{aligned}
& L\left(1_{3 / 2}^{-}\right)=\sum_{\lambda} \varepsilon^{(\lambda) \nu} \varepsilon^{\prime(\lambda) * \sigma} \frac{3}{2} \operatorname{Tr}\left\{\overline{\mathcal{B}}_{f} \bar{\Gamma}_{2} P_{+}^{\prime}\left[v_{f \sigma}-\frac{1}{3} \gamma_{\sigma}\left(\psi_{f}+w_{f}\right)\right]\right\} \\
& \operatorname{Tr}\left\{\left[v_{i \nu}-\frac{1}{3}\left(\psi_{i}+w_{i}\right) \gamma_{\nu}\right] P_{+}^{\prime} \Gamma_{1} \mathcal{B}_{i}\right\} 3 \sum_{n} \sigma_{3 / 2}^{(n)}\left(w_{i}\right) \sigma_{3 / 2}^{(n)}\left(w_{f}\right) .
\end{aligned}
$$


It is convenient to introduce the tensors

$$
\begin{aligned}
& T^{\mu \nu}=\sum_{\lambda} \varepsilon^{\prime(\lambda) \mu} \varepsilon^{\prime(\lambda) * \nu} \\
& T^{\mu \nu, \rho \sigma}=\sum_{\lambda} \varepsilon^{\prime(\lambda) \mu \nu} \varepsilon^{\prime(\lambda) * \rho \sigma} .
\end{aligned}
$$

The polarizations of the vector and tensor intermediate states of velocity $v^{\prime}$ satisfy $\varepsilon^{\prime(\lambda)} \cdot v^{\prime}=\varepsilon^{\prime(\lambda) \mu \nu} v_{\nu}^{\prime}=0$. Moreover, the polarization tensor $\varepsilon^{\prime(\lambda) \mu \nu}$ is symmetric in $(\mu \nu)$ and traceless, $\varepsilon^{\prime(\lambda) \mu}{ }_{\mu}=0$. One can show that these tensors write :

$$
\begin{aligned}
& T^{\mu \nu}=-g^{\mu \nu}+v^{\prime \mu} v^{\prime \nu} \\
& T^{\mu \nu, \rho \sigma}=\frac{1}{6}\left\{-2 g^{\rho \sigma} g^{\mu \nu}+3\left[g^{\rho \mu} g^{\sigma \nu}+g^{\rho \nu} g^{\sigma \mu}\right]+2\left[g^{\rho \sigma} v^{\prime \mu} v^{\prime \nu}+v^{\prime \rho} v^{\prime \sigma} g^{\mu \nu}\right]\right. \\
& \left.-3\left[g^{\rho \mu} v^{\prime \sigma} v^{\prime \nu}+g^{\sigma \nu} v^{\prime \rho} v^{\prime \mu}+g^{\rho \nu} v^{\prime \sigma} v^{\prime \mu}+g^{\sigma \mu} v^{\prime \rho} v^{\prime \nu}\right]+4 v^{\prime \mu} v^{\prime \nu} v^{\rho \rho} v^{\prime \sigma}\right\}
\end{aligned}
$$

and have the following properties : $T^{\mu \nu}$ is symmetric and $T_{\mu}^{\mu}=-3$ while $T^{\mu \nu, \rho \sigma}$ is symmetric in the exchanges $(\mu \nu \leftrightarrow \rho \sigma),(\mu \leftrightarrow \nu)$ and $(\rho \leftrightarrow \sigma)$ and satisfies $T_{\mu \nu}^{\mu \nu}=+5$ (the + sign comes from the fact that the polarization of a spin 2 particle can be seen as a symmetric combination of the polarizations of two spin 1 particles). With these expressions for the polarization tensors one can make more explicit the contributions of the intermediate states in the 1.h.s. of the SR (4). After some algebra one gets, from (26)-(33), for arbitrary Dirac matrices $\Gamma_{1}$ and $\Gamma_{2}$, the following SR

$$
\begin{aligned}
& \left\{\left[\operatorname{Tr}\left[\overline{\mathcal{B}}_{f} \bar{\Gamma}_{2} P_{+}^{\prime} \gamma_{5}\right] \operatorname{Tr}\left[\left(-\gamma_{5}\right) P_{+}^{\prime} \Gamma_{1} \mathcal{B}_{i}\right]\right]\right. \\
& +\left[-\operatorname{Tr}\left[\overline{\mathcal{B}}_{f} \bar{\Gamma}_{2} P_{+}^{\prime} \gamma_{\mu}\right] \operatorname{Tr}\left[\gamma^{\mu} P_{+}^{\prime} \Gamma_{1} \mathcal{B}_{i}\right]\right. \\
& \left.\left.+\operatorname{Tr}\left[\overline{\mathcal{B}}_{f} \bar{\Gamma}_{2} P_{+}^{\prime}\right] \operatorname{Tr}\left[P_{+}^{\prime} \Gamma_{1} \mathcal{B}_{i}\right]\right]\right\} \sum_{n} \xi^{(n)}\left(w_{i}\right) \xi^{(n)}\left(w_{f}\right) \\
& +\frac{1}{2}\left\{\left[3\left(w_{i f}-w_{f} w_{i}\right) \operatorname{Tr}\left[\overline{\mathcal{B}}_{f} \bar{\Gamma}_{2} P_{+}^{\prime} \gamma_{\sigma}\right] \operatorname{Tr}\left[\gamma^{\sigma} P_{+}^{\prime} \Gamma_{1} \mathcal{B}_{i}\right]\right.\right. \\
& +\left(-2-2 w_{i}-2 w_{f}-3 w_{i f}+4 w_{i} w_{f}\right) \operatorname{Tr}\left[\overline{\mathcal{B}}_{f} \bar{\Gamma}_{2} P_{+}^{\prime}\right] \operatorname{Tr}\left[P_{+}^{\prime} \Gamma_{1} \mathcal{B}_{i}\right] \\
& +3 \operatorname{Tr}\left[\overline{\mathcal{B}}_{f} \bar{\Gamma}_{2} P_{+}^{\prime} \psi_{i}\right] \operatorname{Tr}\left[\psi_{f} P_{+}^{\prime} \Gamma_{1} \mathcal{B}_{i}\right]-3 w_{i} \operatorname{Tr}\left[\overline{\mathcal{B}}_{f} \bar{\Gamma}_{2} P_{+}^{\prime}\right] \operatorname{Tr}\left[\psi_{f} P_{+}^{\prime} \Gamma_{1} \mathcal{B}_{i}\right] \\
& \left.-3 w_{f} \operatorname{Tr}\left[\overline{\mathcal{B}}_{f} \bar{\Gamma}_{2} P_{+}^{\prime} \psi_{i}\right] \operatorname{Tr}\left[P_{+}^{\prime} \Gamma_{1} \mathcal{B}_{i}\right]\right] \\
& +\left[-\left(1+w_{i}\right)\left(1+w_{f}\right) \operatorname{Tr}\left[\overline{\mathcal{B}}_{f} \bar{\Gamma}_{2} P_{+}^{\prime} \gamma_{5} \gamma_{\sigma}\right] \operatorname{Tr}\left[\gamma^{\sigma}\left(-\gamma_{5}\right) P_{+}^{\prime} \Gamma_{1} \mathcal{B}_{i}\right]\right. \\
& +\left(1-9 w_{i f}+4 w_{i} w_{f}-2 w_{i}-2 w_{f}\right) \operatorname{Tr}\left[\overline{\mathcal{B}}_{f} \bar{\Gamma}_{2} P_{+}^{\prime} \gamma_{5}\right] \operatorname{Tr}\left[\left(-\gamma_{5}\right) P_{+}^{\prime} \Gamma_{1} \mathcal{B}_{i}\right]
\end{aligned}
$$




$$
\begin{aligned}
& -3\left(1+w_{f}\right) \operatorname{Tr}\left[\overline{\mathcal{B}}_{f} \bar{\Gamma}_{2} P_{+}^{\prime} \gamma_{5} \psi_{i}\right] \operatorname{Tr}\left[\left(-\gamma_{5}\right) P_{+}^{\prime} \Gamma_{1} \mathcal{B}_{i}\right] \\
& \left.\left.-3\left(1+w_{i}\right) \operatorname{Tr}\left[\overline{\mathcal{B}}_{f} \bar{\Gamma}_{2} P_{+}^{\prime} \gamma_{5}\right] \operatorname{Tr}\left[\psi_{f}\left(-\gamma_{5}\right) P_{+}^{\prime} \Gamma_{1} \mathcal{B}_{i}\right]\right]\right\} \sum_{n} \tau_{3 / 2}^{(n)}\left(w_{i}\right) \tau_{3 / 2}^{(n)}\left(w_{f}\right) \\
& +4\left\{\left[\operatorname{Tr}\left[\overline{\mathcal{B}}_{f} \bar{\Gamma}_{2} P_{+}^{\prime}\right] \operatorname{Tr}\left[P_{+}^{\prime} \Gamma_{1} \mathcal{B}_{i}\right]\right]\right. \\
& +\left[-\operatorname{Tr}\left[\overline{\mathcal{B}}_{f} \bar{\Gamma}_{2} P_{+}^{\prime} \gamma_{5} \gamma_{\sigma}\right] \operatorname{Tr}\left[\gamma^{\sigma}\left(-\gamma_{5}\right) P_{+}^{\prime} \Gamma_{1} \mathcal{B}_{i}\right]\right. \\
& \left.\left.+\operatorname{Tr}\left[\overline{\mathcal{B}}_{f} \bar{\Gamma}_{2} P_{+}^{\prime} \gamma_{5}\right] \operatorname{Tr}\left[\left(-\gamma_{5}\right) P_{+}^{\prime} \Gamma_{1} \mathcal{B}_{i}\right]\right]\right\} \sum_{n} \tau_{1 / 2}^{(n)}\left(w_{i}\right) \tau_{1 / 2}^{(n)}\left(w_{f}\right) \\
& +\frac{1}{2}\left\{\left[3\left(w_{i f}-w_{f} w_{i}\right) \operatorname{Tr}\left[\overline{\mathcal{B}}_{f} \bar{\Gamma}_{2} P_{+}^{\prime} \gamma_{\sigma}\left(-\gamma_{5}\right)\right] \operatorname{Tr}\left[\gamma_{5} \gamma^{\sigma} P_{+}^{\prime} \Gamma_{1} \mathcal{B}_{i}\right]\right.\right. \\
& +\left(-2+2 w_{i}+2 w_{f}-3 w_{i f}+4 w_{i} w_{f}\right) \operatorname{Tr}\left[\overline{\mathcal{B}}_{f} \bar{\Gamma}_{2} P_{+}^{\prime}\left(-\gamma_{5}\right)\right] \operatorname{Tr}\left[\gamma_{5} P_{+}^{\prime} \Gamma_{1} \mathcal{B}_{i}\right] \\
& +3 \operatorname{Tr}\left[\overline{\mathcal{B}}_{f} \bar{\Gamma}_{2} P_{+}^{\prime} \psi_{i}\left(-\gamma_{5}\right)\right] \operatorname{Tr}\left[\gamma_{5} \psi_{f} P_{+}^{\prime} \Gamma_{1} \mathcal{B}_{i}\right] \\
& -3 w_{i} \operatorname{Tr}\left[\overline{\mathcal{B}}_{f} \bar{\Gamma}_{2} P_{+}^{\prime}\left(-\gamma_{5}\right)\right] \operatorname{Tr}\left[\gamma_{5} \psi_{f} P_{+}^{\prime} \Gamma_{1} \mathcal{B}_{i}\right] \\
& \left.-3 w_{f} \operatorname{Tr}\left[\overline{\mathcal{B}}_{f} \bar{\Gamma}_{2} P_{+}^{\prime} \psi_{i}\left(-\gamma_{5}\right)\right] \operatorname{Tr}\left[\gamma_{5} P_{+}^{\prime} \Gamma_{1} \mathcal{B}_{i}\right]\right] \\
& +\left[-\left(w_{i}-1\right)\left(w_{f}-1\right) \operatorname{Tr}\left[\overline{\mathcal{B}}_{f} \bar{\Gamma}_{2} P_{+}^{\prime} \gamma_{\sigma}\right] \operatorname{Tr}\left[\gamma^{\sigma} P_{+}^{\prime} \Gamma_{1} \mathcal{B}_{i}\right]\right. \\
& +\left(1-9 w_{i f}+4 w_{i} w_{f}+2 w_{i}+2 w_{f}\right) \operatorname{Tr}\left[\overline{\mathcal{B}}_{f} \bar{\Gamma}_{2} P_{+}^{\prime}\right] \operatorname{Tr}\left[P_{+}^{\prime} \Gamma_{1} \mathcal{B}_{i}\right] \\
& +3\left(w_{f}-1\right) \operatorname{Tr}\left[\overline{\mathcal{B}}_{f} \bar{\Gamma}_{2} P_{+}^{\prime} \psi_{i}\right] \operatorname{Tr}\left[P_{+}^{\prime} \Gamma_{1} \mathcal{B}_{i}\right] \\
& \left.\left.+3\left(w_{i}-1\right) \operatorname{Tr}\left[\overline{\mathcal{B}}_{f} \bar{\Gamma}_{2} P_{+}^{\prime}\right] \operatorname{Tr}\left[\psi_{f} P_{+}^{\prime} \Gamma_{1} \mathcal{B}_{i}\right]\right]\right\} \sum_{n} \sigma_{3 / 2}^{(n)}\left(w_{i}\right) \sigma_{3 / 2}^{(n)}\left(w_{f}\right)
\end{aligned}
$$

+ Contribution from other excited states

$$
=-2 \xi\left(w_{i f}\right) \operatorname{Tr}\left[\overline{\mathcal{B}}_{f}\left(v_{f}\right) \bar{\Gamma}_{2} P_{+}^{\prime} \Gamma_{1} \mathcal{B}_{i}\left(v_{i}\right)\right] .
$$

In the r.h.s., the function $\xi\left(w_{i f}\right)$ must match the corresponding function of $w_{i f}$ that one would get summing over all possible intermediate states. In this formula, the coefficient of $\sum_{n} \xi^{(n)}\left(w_{i}\right) \xi^{(n)}\left(w_{f}\right)$ is the contribution of the $0_{1 / 2}^{-}$(first bracket) and the $1_{1 / 2}^{-}$(second bracket) states. Likewise, the coefficient of $\sum_{n} \tau_{3 / 2}^{(n)}\left(w_{i}\right) \tau_{3 / 2}^{(n)}\left(w_{f}\right)$ is the contribution of the $2_{3 / 2}^{+}$(first bracket) and the $1_{3 / 2}^{+}$states (second bracket). The coefficient of $\sum_{n} \tau_{1 / 2}^{(n)}\left(w_{i}\right) \tau_{1 / 2}^{(n)}\left(w_{f}\right)$ is the contribution of the $0_{1 / 2}^{+}$(first bracket) and the $1_{1 / 2}^{+}$states (second bracket). Finally, the coefficient of $\sum_{n} \sigma_{3 / 2}^{(n)}\left(w_{i}\right) \sigma_{3 / 2}^{(n)}\left(w_{f}\right)$ is the contribution of the $2_{3 / 2}^{-}$(first bracket) and the $1_{3 / 2}^{-}$(second bracket).

What we did call $L\left(w_{i f}, w_{i}, w_{f}\right)$ and $R\left(w_{i f}, w_{i}, w_{f}\right)$ in Section 1 are given now explicitely by (38). We will now consider the sum rules given by (14). However, since we have included only a limited number of intermediate states, it would be dangerous to draw conclusions from sum rules for $p, q, r \geq 2$, because missing intermediate states could contribute to the desired order. Therefore, we will limit ourselves to 
$(p, q, r)=(0,0,0),(1,0,0),(0,1,0),(0,0,1)$. The consideration of the states $\frac{3}{2}^{-}$will give us some control over higher powers of $(w-1)$. In the main text, we will limit ourselves to currents that give functions $L\left(w_{i f}, w_{i}, w_{f}\right)$ and $R\left(w_{i f}, w_{i}, w_{f}\right)$ symmetric in $w_{i}, w_{f}$. We are then limited to the following relations from the different derivatives and boundary conditions :

$$
\begin{gathered}
\left.L\left(w_{i f}, w_{i}, w_{f}\right)\right|_{w_{i f}=1, w_{i}=w_{f}=w}=\left.R\left(w_{i f}, w_{i}, w_{f}\right)\right|_{w_{i f}=1, w_{i}=w_{f}=w} \\
\left.L\left(w_{i f}, w_{i}, w_{f}\right)\right|_{w_{i}=1, w_{i f}=w_{f}=w}=\left.R\left(w_{i f}, w_{i}, w_{f}\right)\right|_{w_{i}=1, w_{i f}=w_{f}=w} \\
\left.\frac{\partial L}{\partial w_{i f}}\right|_{w_{i f}=1, w_{i}=w_{f}=w}=\left.\frac{\partial R}{\partial w_{i f}}\right|_{w_{i f}=1, w_{i}=w_{f}=w} \\
=\left.\left.\frac{\partial R}{\partial L}\right|_{i w_{i f}}\right|_{w_{i}=1, w_{i f}=w_{f}=w} \\
=\left.\frac{\partial R}{\partial w_{i}}\right|_{w_{i}=1, w_{i f}=w_{f}=w} \\
\left.\frac{\partial L}{\partial w_{i}}\right|_{w_{i}=1, w_{i f}=w_{f}=w}=\left.\frac{\partial R}{\partial w_{i}}\right|_{w_{f}=1, w_{i}=w_{f}=w_{i f}=w} \\
\left.\frac{\partial L}{\partial w_{i}}\right|_{w_{f}=1, w_{i}=w_{i f}=w}=\left.\frac{\partial R}{\partial w_{i}}\right|_{w_{i f}=1, w_{i}=w_{f}=w} \\
\left.\frac{\partial L}{\partial w_{i}}\right|_{w_{i f}=1, w_{i}=w_{f}=w}
\end{gathered}
$$

In Appendix B, we will consider a manifestly covariant version of Uraltsev case, where the functions $L\left(w_{i f}, w_{i}, w_{f}\right), R\left(w_{i f}, w_{i}, w_{f}\right)$ are not symmetric in $w_{i}, w_{f}$. In our conclusion we discuss the perspectives and outlook of these non-symmetric cases.

\section{The axial current : a simple covariant deriva- tion of Bjorken of Uraltsev sum rules.}

To illustrate the method, let us now particularize to the simple case :

$$
\begin{array}{ll}
\mathcal{B}_{i}=P_{i+}\left(-\gamma_{5}\right) & \mathcal{B}_{f}=P_{f+}\left(-\gamma_{5}\right) \\
\Gamma_{1}=\psi_{i} \gamma_{5} & \Gamma_{2}=\psi_{f} \gamma_{5} .
\end{array}
$$


where the currents are projected along the initial and final velocities.

In this symmetric situation between currents and initial and final states, a number of intermediate states do not contribute, and the calculation simplifies considerably. One has, namely :

$$
L\left(0_{1 / 2}^{-}\right)=L\left(1_{3 / 2}^{+}\right)=L\left(1_{1 / 2}^{+}\right)=L\left(2_{3 / 2}^{-}\right)=0
$$

and the SR (38) writes

$$
\begin{aligned}
& \left(w_{i} w_{f}-w_{i f}\right) \sum_{n} \xi^{(n)}\left(w_{i}\right) \xi^{(n)}\left(w_{f}\right) \\
& +\left[3\left(w_{i} w_{f}-w_{i f}\right)^{2}-\left(w_{i}^{2}-1\right)\left(w_{f}^{2}-1\right)\right] \sum_{n} \tau_{3 / 2}^{(n)}\left(w_{i}\right) \tau_{3 / 2}^{(n)}\left(w_{f}\right) \\
& +4\left(w_{i}-1\right)\left(w_{f}-1\right) \sum_{n} \tau_{1 / 2}^{(n)}\left(w_{i}\right) \tau_{1 / 2}^{(n)}\left(w_{f}\right) \\
& +2\left(w_{i}-1\right)\left(w_{f}-1\right)\left(w_{i} w_{f}-w_{i f}\right) \sum_{n} \sigma_{3 / 2}^{(n)}\left(w_{i}\right) \sigma_{3 / 2}^{(n)}\left(w_{f}\right)
\end{aligned}
$$

+ Contribution from other excited states

$$
=-\left(1-w_{i}-w_{f}+w_{i f}\right) \xi\left(w_{i f}\right)
$$

The symmetry of $(48)$ in $\left(w_{i}, w_{f}\right)$ follows from the symmetric choice (46) of currents and states.

We assume now that the higher states contributions are, at most, of the same order in $(w-1)$ as the $\frac{3}{2}^{-}$states, that are included in the calculation. This conjecture will be demonstrated in Section 5. The equations (40), (42) and (44) are trivial (giving $0=0$, or $\xi(w)=\xi(w)$ ), while (39), (41), (43) and (45) give, respectively (the contribution of higher excited states is denoted by $+\cdots$ ) :

$$
\begin{aligned}
& \quad\left(w^{2}-1\right) \sum_{n}\left[\xi^{(n)}(w)\right]^{2}+2\left(w^{2}-1\right)^{2} \sum_{n}\left[\tau_{3 / 2}^{(n)}(w)\right]^{2} \\
& +4(w-1)^{2} \sum_{n}\left[\tau_{3 / 2}^{(n)}(w)\right]^{2}+2(w+1)(w-1)^{3} \sum_{n}\left[\sigma_{3 / 2}^{(n)}(w)\right]^{2}+\cdots \\
& =2(w-1) \\
& -\sum_{n}\left[\xi^{(n)}(w)\right]^{2}-6\left(w^{2}-1\right) \sum_{n}\left[\tau_{3 / 2}^{(n)}(w)\right]^{2}-2(w-1)^{2} \sum_{n}\left[\sigma_{3 / 2}^{(n)}(w)\right]^{2}+\cdots \\
& =-1-2 \rho^{2}(w-1) \\
& 2(w+1) \sum_{n} \tau_{3 / 2}^{(n)}(1) \tau_{3 / 2}^{(n)}(w)-4 \sum_{n} \tau_{1 / 2}^{(n)}(1) \tau_{1 / 2}^{(n)}(w)+\cdots=\xi(w)
\end{aligned}
$$




$$
\begin{aligned}
& w \sum_{n}\left[\xi^{(n)}(w)\right]^{2}+\left(w^{2}-1\right) \sum_{n} \xi^{(n)}(w) \xi^{(n)^{\prime}}(w) \\
& +2\left(w^{2}-1\right)\left\{2 w \sum_{n}\left[\tau_{3 / 2}^{(n)}(w)\right]^{2}+\left(w^{2}-1\right) \sum_{n} \tau_{3 / 2}^{(n)}(w) \tau_{3 / 2}^{(n)^{\prime}}(w)\right\} \\
& +4(w-1)\left\{\sum_{n}\left[\tau_{1 / 2}^{(n)}(w)\right]^{2}+(w-1) \sum_{n} \tau_{1 / 2}^{(n)}(w) \tau_{1 / 2}^{(n)^{\prime}}(w)\right\} \\
& +2(w-1)^{2}\left\{(2 w+1) \sum_{n}\left[\sigma_{3 / 2}^{(n)}(w)\right]^{2}+\left(w^{2}-1\right) \sum_{n} \sigma_{3 / 2}^{(n)}(w) \sigma_{3 / 2}^{(n)^{\prime}}(w)\right\}+\cdots \\
& =1
\end{aligned}
$$

Dividing (49) by 2(w-1) one gets Bjorken SR [1] :

$$
\begin{aligned}
& \frac{w+1}{2} \sum_{n}\left[\xi^{(n)}(w)\right]^{2}+(w-1)\left\{2 \sum_{n}\left[\tau_{1 / 2}^{(n)}(w)\right]^{2}+(w+1)^{2} \sum_{n}\left[\tau_{3 / 2}^{(n)}(w)\right]^{2}\right\} \\
& +(w+1)(w-1)^{2} \sum_{n}\left[\sigma_{3 / 2}^{(n)}(w)\right]^{2}+\cdots=1 .
\end{aligned}
$$

where the $\frac{3}{2}^{-}$states have been included explicitly.

Equation (50) gives, at order $(w-1)$ :

$$
1-2 \rho^{2}(w-1)+12 \sum_{n}\left[\tau_{3 / 2}^{(n)}(1)\right]^{2}(w-1)=1+2 \rho^{2}(w-1)
$$

implying :

$$
\rho^{2}=3 \sum_{n}\left[\tau_{3 / 2}^{(n)}(1)\right]^{2}
$$

that, combined with the first order in $(w-1)$ of Bjorken SR (53)

$$
\rho^{2}=\frac{1}{4}+\sum_{n}\left[\tau_{1 / 2}^{(n)}(1)\right]^{2}+2 \sum_{n}\left[\tau_{3 / 2}^{(n)}(1)\right]^{2}
$$

gives Uraltsev SR [6] :

$$
\sum_{n}\left[\tau_{3 / 2}^{(n)}(1)\right]^{2}-\sum_{n}\left[\tau_{1 / 2}^{(n)}(1)\right]^{2}=\frac{1}{4}
$$

Equation (51) yields also Uraltsev SR for $w=1$. Notice the important point that in this equation the contribution of the IW functions $\sigma_{3 / 2}^{(n)}(w)$ vanishes identically.

Finally, equation $(52)$ at $O[(w-1)]$ gives again Bjorken SR under the form (56). 


\section{The case of the vector current.}

Let us now consider the vector current, i.e.

$$
\begin{array}{rlrl}
\mathcal{B}_{i} & =P_{i+}\left(-\gamma_{5}\right) & & \mathcal{B}_{f}=P_{f+}\left(-\gamma_{5}\right) \\
\Gamma_{1}=\psi_{i} & & \Gamma_{2}=\psi_{f} .
\end{array}
$$

In this particular case, a number of different intermediate states do not contribute, namely :

$$
L\left(1_{1 / 2}^{-}\right)=L\left(2_{3 / 2}^{+}\right)=L\left(0_{1 / 2}^{+}\right)=L\left(1_{3 / 2}^{-}\right)=0
$$

and the SR (38) writes :

$$
\begin{aligned}
& \left(w_{i}+1\right)\left(w_{f}+1\right) \sum_{n} \xi^{(n)}\left(w_{i}\right) \xi^{(n)}\left(w_{f}\right) \\
& +2\left(w_{i}+1\right)\left(w_{f}+1\right)\left(w_{i} w_{f}-w_{i f}\right) \sum_{n} \tau_{3 / 2}^{(n)}\left(w_{i}\right) \tau_{3 / 2}^{(n)}\left(w_{f}\right) \\
& +4\left(w_{i} w_{f}-w_{i f}\right) \sum_{n} \tau_{1 / 2}^{(n)}\left(w_{i}\right) \tau_{1 / 2}^{(n)}\left(w_{f}\right) \\
& +\left[3\left(w_{i} w_{f}-w_{i f}\right)^{2}-\left(w_{i}^{2}-1\right)\left(w_{f}^{2}-1\right)\right] \sum_{n} \sigma_{3 / 2}^{(n)}\left(w_{i}\right) \sigma_{3 / 2}^{(n)}\left(w_{f}\right)
\end{aligned}
$$

+ Contribution from other excited states

$$
=\left(w_{i f}+1+w_{f}+w_{i}\right) \xi\left(w_{i f}\right)
$$

where the first, second, third and fourth term in the r.h.s. comes from the states $0_{1 / 2}^{-}, 1_{3 / 2}^{+}, 1_{1 / 2}^{+}$and $2_{3 / 2}^{-}$, respectively.

Equation (40) is trivial $(\xi(w)=\xi(w))$, while equations (39), (41)-(45) give now, respectively :

$$
\begin{gathered}
(w+1)^{2} \sum_{n}\left[\xi^{(n)}(w)\right]^{2}+2\left(w^{2}-1\right)\left\{(w+1)^{2} \sum_{n}\left[\tau_{3 / 2}^{(n)}(w)\right]^{2}+2 \sum_{n}\left[\tau_{1 / 2}^{(n)}(w)\right]^{2}\right\} \\
+2\left(w^{2}-1\right)^{2} \sum_{n}\left[\sigma_{3 / 2}^{(n)}(w)\right]^{2}+\cdots=2(w+1) \\
\quad-2(w+1)^{2} \sum_{n}\left[\tau_{3 / 2}^{(n)}(w)\right]^{2}-4 \sum_{n}\left[\tau_{1 / 2}^{(n)}(w)\right]^{2} \\
\quad-6\left(w^{2}-1\right) \sum_{n}\left[\sigma_{3 / 2}^{(n)}(w)\right]^{2}+\cdots=1-2(w+1) \rho^{2} \\
-4(w+1) \sum_{n} \tau_{3 / 2}^{(n)}(1) \tau_{3 / 2}^{(n)}(w)-4 \sum_{n} \tau_{1 / 2}^{(n)}(1) \tau_{1 / 2}^{(n)}(w)+\cdots \\
=\xi(w)+2(w+1) \xi^{\prime}(w)
\end{gathered}
$$




$$
\begin{gathered}
w \xi(w)+2(w+1) \sum_{n} \xi^{(n)^{\prime}}(1) \xi^{(n)}(w) \\
+4 w(w+1) \sum_{n} \tau_{3 / 2}^{(n)}(1) \tau_{3 / 2}^{(n)}(w)+4 w \sum_{n} \tau_{1 / 2}^{(n)}(1) \tau_{1 / 2}^{(n)}(w) \\
-2\left(w^{2}-1\right) \sum_{n} \sigma_{3 / 2}^{(n)}(1) \sigma_{3 / 2}^{(n)}(w)+\cdots=0 \\
\xi(w)+2(w+1) \xi^{\prime}(w) \\
+4(w+1) \sum_{n} \tau_{3 / 2}^{(n)}(1) \tau_{3 / 2}^{(n)}(w)+4 \sum_{n} \tau_{1 / 2}^{(n)}(1) \tau_{1 / 2}^{(n)}(w)+\cdots=0 \\
(w+1) \sum_{n}\left[\xi^{(n)}(w)\right]^{2}+(w+1)^{2} \sum_{n} \xi^{(n)}(w) \xi^{(n)^{\prime}}(w) \\
+2(w+1)^{2}\left\{(2 w-1) \sum_{n}\left[\tau_{3 / 2}^{(n)}(w)\right]^{2}+\left(w^{2}-1\right) \sum_{n} \tau_{3 / 2}^{(n)}(w) \tau_{3 / 2}^{(n)^{\prime}}(w)\right\} \\
+4\left\{w \sum_{n}\left[\tau_{1 / 2}^{(n)}(w)\right]^{2}+\left(w^{2}-1\right) \sum_{n} \tau_{1 / 2}^{(n)}(w) \tau_{1 / 2}^{(n)^{\prime}}(w)\right\} \\
+\left(w^{2}-1\right)\left\{4 w \sum_{n}\left[\sigma_{3 / 2}^{(n)}(w)\right]^{2}+2\left(w^{2}-1\right) \sum_{n} \sigma_{3 / 2}^{(n)}(w) \sigma_{3 / 2}^{(n)^{\prime}}(w)\right\}+\cdots=1
\end{gathered}
$$

Notice an important point, namely that in equation (63), identical to equation (65), the contribution of the IW functions $\sigma_{3 / 2}^{(n)}(w)$ vanishes identically.

Dividing (61) by $2(w+1)$ one gets Bjorken SR for all $w(53)$. Equations (62)-(66) imply, for $w=1$, Bjorken SR (56) for the elastic slope $\rho^{2}$.

\section{A new class of sum rules and the contribution of higher excited states.}

Among the SR that we have obtained in Sections 3 and 4, there is a new class that involves the IW functions $\xi^{(n)}(w), \tau_{3 / 2}^{(n)}(w), \tau_{1 / 2}^{(n)}(w), \ldots$ for any $w$ and at zero recoil $w=1$. The relation that we got from the axial currents is :

$$
2(w+1) \sum_{n} \tau_{3 / 2}^{(n)}(1) \tau_{3 / 2}^{(n)}(w)-4 \sum_{n} \tau_{1 / 2}^{(n)}(1) \tau_{1 / 2}^{(n)}(w)+\cdots=\xi(w)
$$


while we obtained, from the vector current :

$$
\begin{gathered}
-4(w+1) \sum_{n} \tau_{3 / 2}^{(n)}(1) \tau_{3 / 2}^{(n)}(w)-4 \sum_{n} \tau_{1 / 2}^{(n)}(1) \tau_{1 / 2}^{(n)}(w)+\cdots=\xi(w)+2(w+1) \xi^{\prime}(w) \\
w \xi(w)+2(w+1) \sum_{n} \xi^{(n)^{\prime}}(1) \xi^{(n)}(w) \\
+4 w(w+1) \sum_{n} \tau_{3 / 2}^{(n)}(1) \tau_{3 / 2}^{(n)}(w)+4 w \sum_{n} \tau_{1 / 2}^{(n)}(1) \tau_{1 / 2}^{(n)}(w) \\
-2\left(w^{2}-1\right) \sum_{n} \sigma_{3 / 2}^{(n)}(1) \sigma_{3 / 2}^{(n)}(w)+\cdots=0
\end{gathered}
$$

The first equation (67) is a generalization of Uraltsev SR for $w \neq 1$, that reduces to (57) for $w=1$, while the other two (68) and (69) give, taking $w=1$, Bjorken SR (56) for the slope $\rho^{2}$.

Let us concentrate on equations (67) and (68). An important feature of these relations is that the contribution from the $\frac{3}{2}^{-}$states vanishes identically. This is not the case however for relation (69).

We will now give a proof that no other higher intermediate states contribute to the sum rules (67) and (68).

Following the work of A. Falk [16], we write first the $4 \times 4$ matrices of the whole tower of $j^{P}$ states, generalizing the notation we have given above (17)-(21), where $k=j-\frac{1}{2}, J$ is the spin of the state, and $\ell$ is the orbital angular momentum :

$j=\ell+\frac{1}{2}, J=j+\frac{1}{2}:$

$$
\mathcal{M}^{\mu_{1} \cdots \mu_{k}}(v)=P_{+} \varepsilon_{v}^{\mu_{1} \cdots \mu_{k+1}} \gamma_{\mu_{k+1}}
$$

$j=\ell+\frac{1}{2}, J=j-\frac{1}{2}:$

$$
\begin{gathered}
\mathcal{M}^{\mu_{1} \cdots \mu_{k}}(v)=-\sqrt{\frac{2 k+1}{k+1}} P_{+} \gamma_{5} \varepsilon_{v}^{\nu_{1} \cdots \nu_{k}} \\
{\left[g_{\nu_{1}}^{\mu_{1}} \cdots g_{\nu_{k}}^{\mu_{k}}-\frac{1}{2 k+1} \gamma_{\nu_{1}}\left(\gamma^{\mu_{1}}-v^{\mu_{1}}\right) g_{\nu_{2}}^{\mu_{2}} \cdots g_{\nu_{k}}^{\mu_{k}}-\cdots-\frac{1}{2 k+1} g_{\nu_{1}}^{\mu_{1}} \cdots g_{\nu_{k-1}}^{\mu_{k-1}} \gamma_{\nu_{k}}\left(\gamma^{\mu_{k}}-v^{\mu_{k}}\right)\right]} \\
j=\ell-\frac{1}{2}, J=j+\frac{1}{2}: \\
\mathcal{M}^{\mu_{1} \cdots \mu_{k}}(v)=P_{+} \varepsilon_{v}^{\mu_{1} \cdots \mu_{k+1}} \gamma_{5} \gamma_{\mu_{k+1}} \\
j=\ell-\frac{1}{2}, J=j-\frac{1}{2}:
\end{gathered}
$$




$$
\begin{gathered}
\mathcal{M}^{\mu_{1} \cdots \mu_{k}}(v)=\sqrt{\frac{2 k+1}{k+1}} P_{+} \varepsilon^{\nu_{1} \cdots \nu_{k}} \\
{\left[g_{\nu_{1}}^{\mu_{1}} \cdots g_{\nu_{k}}^{\mu_{k}}-\frac{1}{2 k+1} \gamma_{\nu_{1}}\left(\gamma^{\mu_{1}}+v^{\mu_{1}}\right) g_{\nu_{2}}^{\mu_{2}} \cdots g_{\nu_{k}}^{\mu_{k}}-\cdots-\frac{1}{2 k+1} g_{\nu_{1}}^{\mu_{1}} \cdots g_{\nu_{k-1}}^{\mu_{k-1}} \gamma_{\nu_{k}}\left(\gamma^{\mu_{k}}+v^{\mu_{k}}\right)\right]}
\end{gathered}
$$

For a transition of the type $\mathcal{B}^{\mu_{1} \cdots \mu_{k}}(v) \rightarrow \mathcal{D}^{\nu_{1} \cdots \nu_{k^{\prime}}}\left(v^{\prime}\right)$, the preceding expressions have to be contracted with the tensor containing all possible independent IW functions $\left(k^{\prime} \geq k\right)$ :

$$
\begin{aligned}
& \xi_{\nu_{1} \cdots \nu_{k^{\prime}}, \mu_{1} \cdots \mu_{k}}=(-1)^{k}\left(v-v^{\prime}\right)_{\nu_{k+1}} \cdots\left(v-v^{\prime}\right)_{\nu_{k^{\prime}}}\left[\xi_{0}^{\left(k^{\prime}, k\right)}(w) g_{\nu_{1} \mu_{1}} \cdots g_{\nu_{k} \mu_{k}}\right. \\
& +\xi_{1}^{\left(k^{\prime}, k\right)}(w)\left(v-v^{\prime}\right)_{\nu_{1}}\left(v-v^{\prime}\right)_{\mu_{1}} g_{\nu_{2} \mu_{2}} \cdots g_{\nu_{k} \mu_{k}}+\cdots \\
& \left.+\xi_{k}^{\left(k^{\prime}, k\right)}(w)\left(v-v^{\prime}\right)_{\nu_{1}}\left(v-v^{\prime}\right)_{\mu_{1}} \cdots\left(v-v^{\prime}\right)_{\nu_{k}}\left(v-v^{\prime}\right)_{\mu_{k}}\right] .
\end{aligned}
$$

However, we are here interested in the transitions between the ground state and the excited states $\frac{1}{2}^{-} \rightarrow j^{P}$, i.e. $k=0$, and the tensor (74) becomes, in this case,

$$
\xi_{\mu_{1} \cdots \mu_{k}}=\left(v-v^{\prime}\right)_{\mu_{1}} \cdots\left(v-v^{\prime}\right)_{\mu_{k}} \xi_{0}^{(k)}(w)
$$

Then, the matrix elements will write, for the different cases :

$$
\begin{gathered}
<D^{(n)}\left(j=\ell+\frac{1}{2}, J=j+\frac{1}{2}\right)\left(v^{\prime}\right)\left|\bar{h}_{v^{\prime}}^{(c)} \Gamma h_{v}^{(b)}\right| B^{(*)}(v)> \\
=\tau_{\ell+\frac{1}{2}}^{(\ell)(n)}(w) v_{\mu_{1}} \cdots v_{\mu_{k}} \varepsilon^{\prime * \mu_{1} \cdots \mu_{k+1}} \operatorname{Tr}\left[\gamma_{\mu_{k+1}} P_{+}^{\prime} \Gamma \mathcal{B}(v)\right] \\
<D^{(n)}\left(j=\ell+\frac{1}{2}, J=j-\frac{1}{2}\right)\left(v^{\prime}\right)\left|\bar{h}_{v^{\prime}}^{(c)} \Gamma h_{v}^{(b)}\right| B^{(*)}(v)>= \\
\sqrt{\frac{2 k+1}{k+1}} \tau_{\ell+\frac{1}{2}}^{(\ell)(n)}(w) \varepsilon^{\prime * \nu_{1} \cdots \nu_{k}} \operatorname{Tr}\left\{\left[v_{\nu_{1}} \cdots v_{\nu_{k}}-\frac{1}{2 k+1}(\psi-w) \gamma_{\nu_{1}} v_{\nu_{2}} \cdots v_{\nu_{k}}-\cdots\right.\right. \\
\left.\left.-\frac{1}{2 k+1} v_{\nu_{1}} \cdots v_{\nu_{k-1}}(\psi-w) \gamma_{\nu_{k}}\right] \gamma_{5} P_{+}^{\prime} \Gamma \mathcal{B}(v)\right\} \\
<D^{(n)}\left({ }_{\left.j=\ell-\frac{1}{2}, J=j+\frac{1}{2}\right)}\left(v^{\prime}\right)\left|\bar{h}_{v^{\prime}}^{(c)} \Gamma h_{v}^{(b)}\right| B^{(*)}(v)>\right. \\
=\tau_{\ell-\frac{1}{2}}^{(\ell)(n)}(w) v_{\mu_{1}} \cdots v_{\mu_{k}} \varepsilon^{\prime * \mu_{1} \cdots \mu_{k+1}} \operatorname{Tr}\left[\gamma_{\mu_{k+1}}\left(-\gamma_{5}\right) P_{+}^{\prime} \Gamma \mathcal{B}(v)\right] \\
<D^{(n)}\left(j=\ell-\frac{1}{2}, J=j-\frac{1}{2}\right)\left(v^{\prime}\right)\left|\bar{h}_{v^{\prime}}^{(c)} \Gamma h_{v}^{(b)}\right| B^{(*)}(v)>=
\end{gathered}
$$




$$
\begin{gathered}
\sqrt{\frac{2 k+1}{k+1}} \tau_{\ell-\frac{1}{2}}^{(\ell)(n)}(w) \varepsilon^{\prime * \nu_{1} \cdots \nu_{k}} \operatorname{Tr}\left\{\left[v_{\nu_{1}} \cdots v_{\nu_{k}}-\frac{1}{2 k+1}(\psi+w) \gamma_{\nu_{1}} v_{\nu_{2}} \cdots v_{\nu_{k}}-\cdots\right.\right. \\
\left.\left.-\frac{1}{2 k+1} v_{\nu_{1}} \cdots v_{\nu_{k-1}}(\psi+w) \gamma_{\nu_{k}}\right] P_{+}^{\prime} \Gamma \mathcal{B}(v)\right\}
\end{gathered}
$$

In all these relations we have made use of the orthogonality condition

$$
v_{\nu_{i}}^{\prime} \varepsilon^{\prime * \nu_{1} \cdots \nu_{k}}=0 \quad(i=1, \cdots, k)
$$

$\mathcal{B}(v)$ denotes the $4 \times 4$ matrix of the ground state, $B$ or $B^{*}(17)$. The functions $\tau_{j=\ell \pm \frac{1}{2}}^{(\ell)(n)}(w)$ are the generalizations to arbitrary $j$ of the IW functions introduced above, namely

$\tau_{1 / 2}^{(0)}(w) \equiv \xi(w), \tau_{3 / 2}^{(1)}(w) \equiv \sqrt{3} \tau_{3 / 2}(w), \tau_{1 / 2}^{(1)}(w) \equiv 2 \tau_{1 / 2}(w), \tau_{3 / 2}^{(2)}(w) \equiv \sqrt{3} \sigma_{3 / 2}(w)$

with an implicit radial quantum number $n$. Therefore, $\tau_{3 / 2}^{(1)}(w)$ and $\tau_{1 / 2}^{(1)}(w)$ are respectively identical to the functions $\tau(w)$ and $\zeta(w)$ defined by Leibovich et al. [4]. The superindex $\ell$ in $\tau_{\ell \pm 1 / 2}^{(\ell)(n)}(w)$ is necessary as indicates the parity, since for a given $j=\ell \pm \frac{1}{2} \geq \frac{1}{2}$, there are two possible values for $\ell=j \pm \frac{1}{2}$, and therefore two possible parities $P=(-1)^{\ell+1}$.

Considering now the $B$ meson, as in the preceding Sections,

$$
\mathcal{B}(v)=P_{+}\left(-\gamma_{5}\right)
$$

we compute the different matrix elements. Remembering that $k=j-\frac{1}{2}$ one obtains the following results.

Vector current :

$$
\begin{aligned}
& <D^{(n)}\left(j=\ell+\frac{1}{2}, J=j+\frac{1}{2}\right)\left(v^{\prime}\right)\left|\bar{h}_{v^{\prime}}^{(c)} \psi h_{v}^{(b)}\right| B(v)> \\
& =<D^{(n)}\left(j=\ell-\frac{1}{2}, J=j-\frac{1}{2}\right)\left(v^{\prime}\right)\left|\bar{h}_{v^{\prime}}^{(c)} \psi h_{v}^{(b)}\right| B(v)>=0 \\
& <D^{(n)}\left(j=\ell+\frac{1}{2}, J=j-\frac{1}{2}\right)\left(v^{\prime}\right)\left|\bar{h}_{v^{\prime}}^{(c)} \psi h_{v}^{(b)}\right| B(v)> \\
& =-\sqrt{\frac{\ell+1}{2 \ell+1}}(w+1) \tau_{\ell+\frac{1}{2}}^{(\ell)(n)}(w) v_{\mu_{1}} \cdots v_{\mu_{\ell}} \varepsilon^{* \mu_{1} \cdots \mu_{\ell}}
\end{aligned}
$$




$$
\begin{aligned}
& \left.<D^{(n)}{ }_{j=\ell-\frac{1}{2}, J=j+\frac{1}{2}}\right)\left(v^{\prime}\right)\left|\bar{h}_{v^{\prime}}^{(c)} \psi h_{v}^{(b)}\right| B(v)> \\
& =-\tau_{\ell-\frac{1}{2}}^{(\ell)(n)}(w) v_{\mu_{1}} \cdots v_{\mu_{\ell}} \varepsilon^{\prime * \mu_{1} \cdots \mu_{\ell}}
\end{aligned}
$$

Axial current :

$$
\begin{aligned}
& <D^{(n)}{ }_{\left(j=\ell+\frac{1}{2}, J=j-\frac{1}{2}\right)\left(v^{\prime}\right)\left|\bar{h}_{v^{\prime}}^{(c)} \psi \gamma_{5} h_{v}^{(b)}\right| B(v)>}=<D^{(n)}{ }_{\left.j=\ell-\frac{1}{2}, J=j+\frac{1}{2}\right)\left(v^{\prime}\right)\left|\bar{h}_{v^{\prime}}^{(c)} \psi \gamma_{5} h_{v}^{(b)}\right| B^{(*)}(v)>=0} \\
& <D^{(n)}\left(j=\ell+\frac{1}{2}, J=j+\frac{1}{2}\right)\left(v^{\prime}\right)\left|\bar{h}_{v^{\prime}}^{(c)} \not \gamma_{5} h_{v}^{(b)}\right| B(v)> \\
& =-\tau_{\ell+\frac{1}{2}}^{(\ell)(n)}(w) v_{\mu_{1}} \cdots v_{\mu_{\ell+1}} \varepsilon^{\prime * \mu_{1} \cdots \mu_{\ell+1}} \\
& <D^{(n)}\left(j=\ell-\frac{1}{2}, J=j-\frac{1}{2}\right)\left(v^{\prime}\right)\left|\bar{h}_{v^{\prime}}^{(c)} \not \gamma_{5} h_{v}^{(b)}\right| B(v)> \\
& =-\sqrt{\frac{\ell}{2 \ell-1}}(w-1) \tau_{\ell-\frac{1}{2}}^{(\ell)(n)}(w) v_{\mu_{1}} \cdots v_{\mu_{\ell-1}} \varepsilon^{* \mu_{1} \cdots \mu_{\ell-1}} .
\end{aligned}
$$

We can now write down the contributions to the l.h.s. of the SR. We proceed as in Sections 3 and 4 adopting the symmetric cases (46) and (58). In an obvious notation, one finds the following results.

Vector current :

$$
\begin{gathered}
L\left(j=\ell+\frac{1}{2}, J=j+\frac{1}{2}\right)=L\left(j=\ell-\frac{1}{2}, J=j-\frac{1}{2}\right)=0 \\
L\left(j=\ell+\frac{1}{2}, J=j-\frac{1}{2}\right)=\frac{\ell+1}{2 \ell+1}\left(w_{i}+1\right)\left(w_{f}+1\right) S_{\ell}\left(w_{i}, w_{f}, w_{i f}\right) \sum_{n} \tau_{\ell+\frac{1}{2}}^{(\ell)(n)}\left(w_{i}\right) \tau_{\ell+\frac{1}{2}}^{(\ell)(n)}\left(w_{f}\right) \\
L\left(j=\ell-\frac{1}{2}, J=j+\frac{1}{2}\right)=S_{\ell}\left(w_{i}, w_{f}, w_{i f}\right) \sum_{n} \tau_{\ell-\frac{1}{2}}^{(\ell)(n)}\left(w_{i}\right) \tau_{\ell-\frac{1}{2}}^{(\ell)(n)}\left(w_{f}\right)
\end{gathered}
$$

Axial current :

$$
L\left(j=\ell+\frac{1}{2}, J=j-\frac{1}{2}\right)=L\left(j=\ell-\frac{1}{2}, J=j+\frac{1}{2}\right)=0
$$




$$
\begin{gathered}
L\left(j=\ell+\frac{1}{2}, J=j+\frac{1}{2}\right)=S_{\ell+1}\left(w_{i}, w_{f}, w_{i f}\right) \sum_{n} \tau_{\ell+\frac{1}{2}}^{(\ell)(n)}\left(w_{i}\right) \tau_{\ell+\frac{1}{2}}^{(\ell)(n)}\left(w_{f}\right) \\
L\left(j=\ell-\frac{1}{2}, J=j-\frac{1}{2}\right)=\frac{\ell}{2 \ell-1}\left(w_{i}-1\right)\left(w_{f}-1\right) S_{\ell-1}\left(w_{i}, w_{f}, w_{i f}\right) \sum_{n} \tau_{\ell-\frac{1}{2}}^{(\ell)(n)}\left(w_{i}\right) \tau_{\ell-\frac{1}{2}}^{(\ell)(n)}\left(w_{f}\right) .
\end{gathered}
$$

In all these relations, the quantity $S_{n}$ defined by

$$
\begin{gathered}
S_{n}=v_{i \nu_{1}} \cdots v_{i \nu_{n}} v_{f \mu_{1}} \cdots v_{f \mu_{n}} T^{\nu_{1} \cdots \nu_{n}, \mu_{1} \cdots \mu_{n}} \\
T^{\nu_{1} \cdots \nu_{n}, \mu_{1} \cdots \mu_{n}}=\sum_{\lambda} \varepsilon^{(\lambda) * \nu_{1} \cdots \nu_{n}} \varepsilon^{(\lambda) \mu_{1} \cdots \mu_{n}}
\end{gathered}
$$

depends only on the four-velocity $v^{\prime}$ and $\varepsilon^{\prime(\lambda) \mu_{1} \cdots \mu_{n}}$ is a symmetric tensor with vanishing contractions and transverse to $v^{\prime}$ (see Appendix A).

It can be shown, as demonstrated below in Appendix A, that the scalar quantity

$$
S_{n}=v_{i \nu_{1}} \cdots v_{i \nu_{n}} v_{f \mu_{1}} \cdots v_{f \mu_{n}} T^{\nu_{1} \cdots \nu_{n}, \mu_{1} \cdots \mu_{n}}
$$

can be computed and is given by the following expression

$$
S_{n}=\sum_{0 \leq k \leq \frac{n}{2}} C_{n, k}\left(w_{i}^{2}-1\right)^{k}\left(w_{f}^{2}-1\right)^{k}\left(w_{i} w_{f}-w_{i f}\right)^{n-2 k}
$$

where

$$
C_{n, k}=(-1)^{k} \frac{(n !)^{2}}{(2 n) !} \frac{(2 n-2 k) !}{k !(n-k) !(n-2 k) !}
$$

We did find that in the SR (51) and (63) or (65) the contribution of the states $\frac{3}{2}^{-}$ is identically zero. Using now the preceding general formulas, let us prove that not only the contribution of the states $j^{P}=\frac{3}{2}^{-}$vanishes, but that this is also the case for any $j \geq \frac{5}{2}$. This result will imply that the SR (67) and (68) are exact equations, i.e. we can drop out the $+\cdots$

Let us begin with equation (51), that was found with the axial current by differentiating with respect to $w_{i}$, and taking the limit $w_{i}=1, w_{f}=w_{i f}=w$. Notice first that

$$
\left.S_{n}\left(w_{i}, w_{f}, w_{i f}\right)\right|_{w_{i}=1, w_{f}=w_{i f}=w}=0 \quad(n \geq 1)
$$

because of the orthogonality condition (80). 
From eqs. (97) and (98) we need to prove that

$$
\begin{gathered}
\left.\frac{\partial}{\partial w_{i}} S_{n+1}\left(w_{i}, w_{f}, w_{i f}\right)\right|_{w_{i}=1, w_{f}=w_{i f}=w}=0 \quad(n \geq 2) \\
\left.\frac{\partial}{\partial w_{i}}\left(w_{i}-1\right)\left(w_{f}-1\right) S_{n}\left(w_{i}, w_{f}, w_{i f}\right)\right|_{w_{i}=1, w_{f}=w_{i f}=w}=0 \quad(n \geq 1) .
\end{gathered}
$$

The second condition (101) is obviously held because of the factor $\left(w_{i}-1\right)$ and the orthogonality condition (80).

The first condition (100) holds also, as can be seen from the explicit formula $(97):$

$$
\begin{gathered}
\frac{\partial}{\partial w_{i}} S_{n+1}\left(w_{i}, w_{f}, w_{i f}\right)=\sum_{0 \leq k \leq \frac{n+1}{2}} C_{n+1, k}\left(w_{f}^{2}-1\right)^{k}\left[2 k w_{i}\left(w_{i}^{2}-1\right)^{k-1}\left(w_{i} w_{f}-w_{i f}\right)^{n+1-2 k}\right. \\
\left.+(n+1-2 k) w_{f}\left(w_{i}^{2}-1\right)^{k}\left(w_{i} w_{f}-w_{i f}\right)^{n-2 k}\right]
\end{gathered}
$$

that vanishes for $w_{i}=1, w_{f}=w_{i f}=w$ when $n \geq 2$. Notice that this expression does not vanish for $n=1$, that corresponds to the contribution of the $\frac{3}{2}^{+}$states to the SR.

Let us now consider equation (63), that was found with the vector current by derivation with respect to $w_{i f}$, and taking the limit $w_{i}=1, w_{f}=w_{i f}=w$, or $(65)$ by derivation with respect to $w_{i}$, and taking the limit $w_{f}=1, w_{i}=w_{i f}=w$. From eq. (97) we need to prove

$$
\begin{gathered}
\left.\frac{\partial}{\partial w_{i f}}\left(w_{i}+1\right)\left(w_{f}+1\right) S_{n}\left(w_{i}, w_{f}, w_{i f}\right)\right|_{w_{i}=1, w_{f}=w_{i f}=w}=0 \quad(n \geq 2) \\
\left.\frac{\partial}{\partial w_{i f}} S_{n+1}\left(w_{i}, w_{f}, w_{i f}\right)\right|_{w_{i}=1, w_{f}=w_{i f}=w}=0 \quad(n \geq 1) .
\end{gathered}
$$

This is indeed the case, since

$$
\frac{\partial}{\partial w_{i f}} S_{n}\left(w_{i}, w_{f}, w_{i f}\right)=-\sum_{0 \leq k \leq \frac{n}{2}} C_{n, k}\left(w_{i}^{2}-1\right)^{k}\left(w_{f}^{2}-1\right)^{k}(n-2 k)\left(w_{i} w_{f}-w_{i f}\right)^{n-2 k-1}
$$


vanishes for $w_{i}=1, w_{f}=w_{i f}=w$ when $n \geq 2$. Notice that this quantity does not vanish for $n=1$, corresponding again to the contribution of the $\frac{3}{2}^{+}$states to the SR. The proof can be done also by derivation with respect to $w_{i}$, and taking the limit $w_{f}=1, w_{i}=w_{i f}=w$.

In conclusion, we have demonstrated that in the SR (67) and (68) there are no contributions from higher excitations.

We must make an important distinction between the different SR that we have obtained. On the one hand, there are the SR to which contribute the whole series of $j^{P}$ excitations. On the other hand, we have obtained two special SR (67) and (68) where only a limited number of intermediate states contribute.

One can understand the truncation of the series in this latter case because the SR correspond to the boundary condition $w_{i}=1, w_{i f}=w_{f}=w$. Therefore, the matrix element $<D^{(n}\left(v^{\prime}\right)\left|\bar{h}_{v^{\prime}}^{(c)} \Gamma_{1} h_{v_{i}}^{(b)}\right| B\left(v_{i}\right)>$ is computed at zero recoil, hence the finite number of terms in the expansion. As we have seen, SR (67) obtained with the axial currents implies at zero recoil Uraltsev SR (57). The corresponding SR from the vector current (68) is the Bjorken-type counterpart and indeed implies, at zero recoil, Bjorken SR for the slope (56).

On the other hand, since all the SR that we have obtained are exact relations, we can derive them relatively to $w$ and, for a given derivative, taking the zero recoil limit $w=1$, the series will be truncated due to the higher powers of the recoil $(w-1)^{\ell}$ as $\ell$ increases. Therefore, one can expect to obtain information on higher derivatives of the elastic IW function $\xi(w)$, as we have done in ref. [15].

\section{A bound on the curvature from the new sum rules.}

In the preceding Section we have demonstrated that the SR (67) and (68) do not have contributions from higher excited states, i.e. we can omit $+\cdots$ in these equations. This is an important result that means that these SR, involving only $\xi(w), \tau_{1 / 2}^{(n)}(w)$ and $\tau_{3 / 2}^{(n)}(w)$, are exact relations for all $w$, namely : 


$$
\begin{gathered}
2(w+1) \sum_{n} \tau_{3 / 2}^{(n)}(1) \tau_{3 / 2}^{(n)}(w)-4 \sum_{n} \tau_{1 / 2}^{(n)}(1) \tau_{1 / 2}^{(n)}(w)=\xi(w) \\
-4(w+1) \sum_{n} \tau_{3 / 2}^{(n)}(1) \tau_{3 / 2}^{(n)}(w)-4 \sum_{n} \tau_{1 / 2}^{(n)}(1) \tau_{1 / 2}^{(n)}(w)=\xi(w)+2(w+1) \xi^{\prime}(w) .
\end{gathered}
$$

These relations are the main result of this paper.

Therefore, we can still differentiate relation (107) relatively to $w$ :

$$
\begin{aligned}
& -4 \sum_{n} \tau_{3 / 2}^{(n)}(1) \tau_{3 / 2}^{(n)}(w)-4(w+1) \sum_{n} \tau_{3 / 2}^{(n)}(1) \tau_{3 / 2}^{(n)^{\prime}}(w) \\
& -4 \sum_{n} \tau_{1 / 2}^{(n)}(1) \tau_{1 / 2}^{(n)^{\prime}}(w)=\xi^{\prime}(w)+2 \xi^{\prime}(w)+2(w+1) \xi^{\prime \prime}(w)
\end{aligned}
$$

expanding the elastic IW function $\xi(w)$ in powers of $(w-1)$,

$$
\xi(w)=1-\rho^{2}(w-1)+\frac{\sigma^{2}}{2}(w-1)^{2}+\cdots
$$

one obtains, at zero recoil

$$
-4 \sum_{n}\left[\tau_{3 / 2}^{(n)}(1)\right]^{2}-8 \sum_{n} \tau_{3 / 2}^{(n)}(1) \tau_{3 / 2}^{(n)^{\prime}}(1)-4 \sum_{n} \tau_{1 / 2}^{(n)}(1) \tau_{1 / 2}^{(n)^{\prime}}(1)=-3 \rho^{2}+4 \sigma^{2}
$$

and from relation (55) for $\rho^{2}$ one obtains

$$
\sigma^{2}=\frac{5}{12} \rho^{2}-2 \sum_{n} \tau_{3 / 2}^{(n)}(1) \tau_{3 / 2}^{(n)^{\prime}}(1)-\sum_{n} \tau_{1 / 2}^{(n)}(1) \tau_{1 / 2}^{(n)^{\prime}}(1) .
$$

We can also differentiate relation (106) relatively to $w$ and take the zero recoil limit :

$$
2 \sum_{n}\left[\tau_{3 / 2}^{(n)}(1)\right]^{2}+4 \sum_{n} \tau_{3 / 2}^{(n)}(1) \tau_{3 / 2}^{(n)^{\prime}}(1)-4 \sum_{n} \tau_{1 / 2}^{(n)}(1) \tau_{1 / 2}^{(n)^{\prime}}(1)=-\rho^{2}
$$

and from (55) we obtain :

$$
\rho^{2}=-\frac{12}{5}\left[\sum_{n} \tau_{3 / 2}^{(n)}(1) \tau_{3 / 2}^{(n)^{\prime}}(1)-\sum_{n} \tau_{1 / 2}^{(n)}(1) \tau_{1 / 2}^{(n)^{\prime}}(1)\right]
$$

Combining relations (111) and (113) one obtains :

$$
\sigma^{2}=-3 \sum_{n} \tau_{3 / 2}^{(n)}(1) \tau_{3 / 2}^{(n)^{\prime}}(1)
$$

Equations (113) and (114) are important results of the present paper. We must insist on the fact that they are exact relations, as no other higher excited states contribute to the sums in the r.h.s. 
Let us now discuss these formulas. If we make the plausible assumption :

$$
-\sum_{n} \tau_{1 / 2}^{(n)}(1) \tau_{1 / 2}^{(n)^{\prime}}(1)>0 .
$$

the following inequality follows from (113) and (114) :

$$
\sigma^{2} \geq \frac{5}{4} \rho^{2}
$$

This inequality, from the lower bound $\rho^{2} \geq \frac{3}{4}$ ([6], [12]), implies the absolute bound

$$
\sigma^{2} \geq \frac{15}{16}
$$

The assumption (115) would be valid if the $n=0$ state dominates the sum, and if $\tau_{1 / 2}^{(0)^{\prime}}(1)<0$. This latter condition is very natural, since it concerns transitions between states of radial quantum number $n=0$, and therefore with no nodes in the wave function.

\section{Phenomenological remarks.}

In the Bakamjian-Thomas type of relativistic quark models, we have shown that Bjorken and Uraltsev SR are satisfied [17]. Moreover, these SR are approximately saturated by the $n=0$ states. We can add that the slopes of all three IW functions $\xi(w), \tau_{3 / 2}^{(0)}(w)$ and $\tau_{1 / 2}^{(0)}(w)$ are negative [18]. Namely, a good approximate parametrization of these functions is given by

$$
\xi(w)=\left(\frac{2}{w+1}\right)^{2 \rho^{2}} \quad, \quad \tau_{3 / 2}^{(0)}(w)=\left(\frac{2}{w+1}\right)^{2 \sigma_{3 / 2}^{2}} \quad, \quad \tau_{1 / 2}^{(0)}(w)=\left(\frac{2}{w+1}\right)^{2 \sigma_{1 / 2}^{2}} .
$$

In the spectroscopic model of Godfrey and Isgur, one finds the results

$$
\begin{array}{rlrl}
\xi(1) & =1 & \rho^{2}=1.02 \\
\tau_{1 / 2}^{(0)}(1) & =0.22 & \sigma_{1 / 2}^{2}=0.83 \\
\tau_{3 / 2}^{(0)}(1) & =0.54 & \sigma_{3 / 2}^{2} & =1.50 .
\end{array}
$$

We observe that approximating the r.h.s. of (113) with the $n=0$ states this SR writes :

$$
\rho^{2}=1.02=0.95+\text { Contributions from } n \neq 0 \text { excitations }
$$


The inequality (116) is satisfied also in the BT scheme, since, for example in the GI spectroscopic model :

$$
\rho^{2} \cong 1
$$

and the inequality (116) writes $3 / 2>5 / 4$. Therefore the conjecture (115) is satisfied in the model. Notice that BT quark models satisfy Bjorken and Uraltsev SR [17].

Although it remains to be proved, it is highly plausible that these models satisfy the whole set of SR of QCD in the heavy quark limit, and therefore the new class (113) and (114).

Finally, from relation (114) we get the following result for the curvature, compared with the direct result (121) from the elastic IW function $\xi(w)(118)$,

$$
\sigma^{2} \cong 1.5=1.31+\text { Contributions from } n \neq 0 \text { excitations }
$$

We can conclude that there is an excellent qualitative agreement between the slope and the curvature of the elastic IW function as given directly from its calculation and as estimated from the SR (113) and (114), if one assumes that the $n=0$ states dominate, as already has been checked from the Bjorken and Uraltsev sum rules.

\section{Conclusions and outlook.}

In conclusion, within the OPE, we have presented a covariant method, using the trace formalism, to obtain sum rules in the heavy quark limit that relate the elastic Isgur-Wise $\xi(w)$ to IW functions of transitions to excited states.

A main ingredient has been the introduction of the domain of the three variables $\left(w_{i}, w_{f}, w_{i f}\right)$, that allows a systematic way of exploring all possible SR. In particular, we have given a simple and direct deduction of Bjorken and Uraltsev SR, with generalizations of the latter for $w \neq 1$. The simplicity of the proof relies on the choice of the pseudoscalar $B$ meson $B\left(v_{i}\right) \rightarrow D^{(n)}\left(v^{\prime}\right) \rightarrow B\left(v_{f}\right)$ and of symmetric currents projected on the initial and final velocities $v_{i}$ and $v_{f}$, like $\left(\Gamma_{1}, \Gamma_{2}\right)=\left(\psi_{i}, \psi_{f}\right)$ or $\left(\psi_{i} \gamma_{5}, \psi_{f} \gamma_{5}\right)$. This simplifies enormously the calculation, since it gives vanishing contributions for half of the possible intermediate states. Notice that we obtain the 
same SR (48) and (60), if we use $\left(\Gamma_{1}, \Gamma_{2}\right)=\left(\not^{\prime}, \not^{\prime}\right)$ or $(\mathbb{1}, \mathbb{1})$ and $\left(\Gamma_{1}, \Gamma_{2}\right)=\left(\not^{\prime} \gamma_{5}, \not^{\prime} \gamma_{5}\right)$ or $\left(i \gamma_{5}, i \gamma_{5}\right)$.

Moreover, a new class of SR, involving on the one hand IW functions at zero recoil and, on the other hand, IW functions for any $w$ have been obtained. These SR reduce to known results for $w=1$.

Among these new SR, we have found two new relations that involve only the elastic IW function $\xi(w)$, and the excited $\tau_{1 / 2}^{(n)}(w)$ and $\tau_{3 / 2}^{(n)}(w)$, with vanishing contributions for all other IW functions between the ground state and higher excited states. The vanishing of the states $\frac{3}{2}^{-}$has been shown explicitely, using the corresponding wave function. We have generalized this result, demonstrating that all contributions of higher states with $j^{ \pm}, j \geq \frac{5}{2}$ vanish identically. An important ingredient in the proof has been a compact formula for the polarization tensor saturated with initial and final four-velocities.

These new SR are therefore very strong and provide new results that relate the slope $\rho^{2}$ and the curvature $\sigma^{2}$ of $\xi(w)$ to $\tau_{1 / 2}^{(n)}(1), \tau_{3 / 2}^{(n)}(1)$ and $\tau_{1 / 2}^{(n)^{\prime}}(1), \tau_{3 / 2}^{(n)^{\prime}}(1)$. Modulo a very natural assumption, these SR imply the bound $\sigma^{2} \geq \frac{5}{4} \rho^{2}$.

On the other hand, as a phenomenological remark, we have shown that these new SR for $\rho^{2}$ and $\sigma^{2}$ are in good agreement with the numerical results obtained within the Bakamjian-Thomas relativistic quark models, that satisfy Isgur-Wise scaling. In this framework, the SR are saturated to a great accuracy by the $n=0$ intermediate states.

Which are the prospects of this work? The main aim would be to obtain all possible usable SR. By usable we mean SR that involve only $\xi(w)$ and $\tau_{1 / 2}^{(n)}(w)$, $\tau_{3 / 2}^{(n)}(w)$.

For the moment, we have concentrated mainly to the case, that appears to be simple, $B\left(v_{i}\right) \rightarrow B\left(v_{f}\right)$ with symmetric currents, projected along $v_{i}$ and $v_{f}$. One should also study, on the one hand, the case of the transitions $B\left(v_{i}\right) \rightarrow B^{*}\left(v_{f}\right)$ and $B^{*}\left(v_{i}\right) \rightarrow B^{*}\left(v_{f}\right)$ and non-symmetric currents like $\left(\Gamma_{1}, \Gamma_{2}\right)=\left(\psi_{i}, \psi_{i}\right),\left(\psi_{i}, \psi^{\prime}\right)$, etc, or equivalently $\left(\Gamma_{1}, \Gamma_{2}\right)=\left(\gamma_{\mu}, \gamma_{\nu}\right),\left(\gamma_{\mu} \gamma_{5}, \gamma_{\nu} \gamma_{5}\right) \ldots$ for which in general all intermediate states contribute. We have explored a number of these non-symmetric situations for the pseudoscalar $B$-meson and found confirmation of the results presented here.

The case of the $B^{*}$ is rather involved because of the polarization, mainly in the 
case of non-symmetric currents, as used by Uraltsev in the finding of his SR. We have given in Appendix B our covariant version of his calculation.

A systematic complete study remains to be done and may be worth. In particular, it would be interesting to check if the conjecture (115) on $\tau_{1 / 2}^{(n)}(w)$, satisfied by BT quark models, that leads from the SR obtained here to $\sigma^{2} \geq \frac{5}{4} \rho^{2}$, is or is not a result of heavy quark symmetry [19].

\section{Appendix A. Projector on the polarization tensors}

The polarization state of a relativistic boson is commonly described by a polarization tensor, generalizing the polarization vector of a spin 1 particle. The polarization tensors of a particle of integer spin $J$ are the tensors $\varepsilon_{\mu_{1} \cdots \mu_{J}}$ of tank $J$ which satisfy the following conditions :

1) Symmetry : $\varepsilon_{\mu_{1} \cdots \mu_{J}}=\varepsilon_{\mu_{\sigma(1)} \cdots \mu_{\sigma(J)}}$ for any permutation $\sigma$ of $1, \cdots, J$.

2) Vanishing contractions (or tracelessness) : $g^{\mu \mu^{\prime}} \varepsilon_{\mu \mu^{\prime} \mu_{3} \cdots \mu_{J}}=0$ (when $J \geq 2$ ).

3) Transversity : $v^{\mu} \varepsilon_{\mu \mu_{2} \cdots \mu_{J}}=0$, where $v$ is the 4-velocity of the particle.

An orthornormal set of $2 J+1$ polarization states will be described by a set $\varepsilon_{\mu_{1} \cdots \mu_{J}}^{(\lambda)}$ of polarization tensors satisfying the following normalisation conditions :

$$
g^{\mu_{1} \nu_{1}} \cdots g^{\mu_{J} \nu_{J}} \varepsilon_{\mu_{1} \cdots \mu_{J}}^{(\lambda)}\left(\varepsilon_{\nu_{1} \cdots \nu_{J}}^{\left(\lambda^{\prime}\right)}\right)^{*}=(-1)^{J} \delta_{\lambda \lambda^{\prime}}
$$

Then, when summing over the intermediate states of a particle of integer spin $J$, one has to deal with the projector on polarization tensors $\prod^{(v)}$ defined by :

$$
\prod_{\mu_{1} \cdots \mu_{J} ; \nu_{1} \cdots \nu_{J}}^{(v)}=\sum_{\lambda=-J}^{J} \varepsilon_{\mu_{1} \cdots \mu_{J}}^{(\lambda)}\left(\varepsilon_{\nu_{1} \cdots \nu_{J}}^{(\lambda)}\right)^{*} .
$$

In this appendix, we intend to deduce an explicit expression for this tensor. The basic result is :

$$
\begin{gathered}
v_{f}^{\mu_{1}} \cdots v_{f}^{\mu_{J}} \prod_{\mu_{1} \cdots \mu_{J} ; \nu_{1} \cdots \nu_{J}}^{(v)} v_{i}^{\nu_{1}} \cdots v_{i}^{\nu_{J}} \\
=2^{J} \frac{(J !)^{2}}{(2 J) !}\left(w_{i}^{2}-1\right)^{J / 2}\left(w_{f}^{2}-1\right)^{J / 2} P_{J}\left(\frac{w_{i} w_{f}-w_{i f}}{\sqrt{\left(w_{i}^{2}-1\right)\left(w_{f}^{2}-1\right)}}\right)
\end{gathered}
$$


where $v_{i}$ and $v_{f}$ are arbitrary velocity 4 -vectors, $w_{i}, w_{f}, w_{i f}$ are the following scalar products :

$$
w_{i}=v \cdot v_{i}, \quad w_{f}=v \cdot v_{f}, \quad w_{i f}=v_{i} \cdot v_{f}
$$

and $P_{n}$ is the usual Legendre polynomial.

The matrix element (A.3) is a polynomial in $w_{i}, w_{f}, w_{i f}$. Using explicit expressions of $P_{n}$, one has the two following useful expressions of this polynomial :

$$
\begin{gathered}
v_{f}^{\mu_{1}} \cdots v_{f}^{\mu_{J}} \prod_{\mu_{1} \cdots \mu_{J} ; \nu_{1} \cdots \nu_{J}}^{(v)} v_{i}^{\nu_{1}} \cdots v_{i}^{\nu_{J}} \\
=\sum_{0 \leq k \leq J / 2} C_{J, k}\left(w_{i}^{2}-1\right)^{k}\left(w_{i} w_{f}-w_{i f}\right)^{J-2 k}\left(w_{f}^{2}-1\right)^{k} \\
v_{f}^{\mu_{1}} \cdots v_{f}^{\mu_{J}} \prod_{\mu_{1} \cdots \mu_{J} ; \nu_{1} \cdots \nu_{J}}^{(v)} v_{i}^{\nu_{1}} \cdots v_{i}^{\nu_{J}} \\
=\sum_{0 \leq k \leq J / 2} C_{J, k}^{\prime}\left(w_{i} w_{f}-w_{i f}\right)^{J-2 k}\left[\left(w_{i}^{2}-1\right)\left(w_{f}^{2}-1\right)-\left(w_{i} w_{f}-w_{i f}\right)^{2}\right]^{k}
\end{gathered}
$$

where the $C_{j, k}$ and $C_{J, k}^{\prime}$ are the numerical coefficients given by :

$$
\begin{gathered}
C_{J, k}=(-1)^{k} \frac{(J !)^{2}}{(2 J) !} \frac{(2 J-2 k) !}{k !(J-k) !(J-2 k) !} \\
C_{J, k}^{\prime}=(-1)^{k} 2^{J-2 k} \frac{(J !)^{2}}{(2 J) !} \frac{J !}{(k !)^{2}(J-2 k) !} .
\end{gathered}
$$

The expression (A.5) is useful when considering the limit $v_{i} \rightarrow v$ in which $w_{i} \rightarrow 1$ and $w_{i f} \rightarrow w_{f}$ (or as well $v_{f} \rightarrow v$ in which $w_{f} \rightarrow 1$ and $w_{i f} \rightarrow w_{i}$ ). The expression (A.6) is useful when considering the limit $v_{f} \rightarrow v_{i}$ in which $w_{i f} \rightarrow 1$ and $w_{f} \rightarrow w_{i}$. Indeed the $k^{\text {th }}$ term in (A.5) or (A.6) vanishes at order $k$, and only the $k=0$ term survives in the considered limit.

In this paper the matrix elements (A.3) are all we need. However, as we shall see the full expression of $\prod^{(v)}$ can be deduced from these particular matrix elements. For brevity, we write this full expression for a particle at rest, in which case only the purely spatial components are non-vanishing. The tensor $\prod_{\mu_{1}, \cdots, \mu_{n} ; \nu_{1}, \cdots, \nu_{n}}^{(v)}$ for an arbitrary 4 -velocity $v$ is readily obtained from the formula below by the substitutions: 


$$
\begin{aligned}
& \delta_{i_{r} i_{r^{\prime}}} \rightarrow-g_{\mu_{r} \mu_{r^{\prime}}}+v_{\mu_{r}} v_{\mu_{r^{\prime}}} \\
& \delta_{i_{s} j_{s^{\prime}}} \rightarrow-g_{\mu_{s} \nu_{s^{\prime}}}+v_{\mu_{s}} v_{\nu_{s^{\prime}}} \\
& \delta_{j_{t} j_{t^{\prime}}} \rightarrow-g_{\nu_{t} \nu_{t^{\prime}}}+v_{\nu_{t}} v_{\nu_{t^{\prime}}} .
\end{aligned}
$$

The formula is :

$$
\begin{gathered}
\prod_{i_{1} \cdots i_{n} ; j_{1} \cdots j_{n}}=\sum_{0 \leq k \leq n / 2} f_{n, k} \sum_{\substack{I, J \in\{1 \cdots n\} \\
|I I|=|J|=2 k}} \\
\left(\sum_{\mathcal{J} \in \mathcal{P}_{2}(I)} \prod_{\left\{r, r^{\prime}\right\} \in \mathcal{J}} \delta_{i_{r} i_{r^{\prime}}}\right)\left(\sum_{\sigma \in \mathcal{B}(\mathbf{C} I, \mathbf{C} J)} \prod_{s \notin I} \delta_{i_{s} j_{\sigma(s)}}\right)\left(\sum_{\substack{\mathcal{J}^{\prime} \in \mathcal{P}_{2}(J) \\
\left\{t, t^{\prime}\right\} \in \mathcal{J}^{\prime}}} \delta_{j_{t} j_{t^{\prime}}}\right)
\end{gathered}
$$

with

$$
f_{n, k}=(-1)^{k} 2^{2 k} \frac{k !(2 n-2 k) !}{(n-k) !(2 n) !} .
$$

In this formula (A.10), $\mathbf{C} I$ and $\mathbf{C} J$ are the complementary sets in $\{1,2, \cdots n\}$ of the subsets $I$ and $J$. For a set $X, \mathcal{P}_{2}(X)$ is the set of partitions of $X$ by two-element subsets (precisely unordered partitions or, partitions as sets of subsets). For two sets $X$ and $Y, \mathcal{B}(X, Y)$ is the set of bijections $X \rightarrow Y$.

(There is a logical subtlety in the (important) terms $I=J=\emptyset$ in (A.10)). Namely, one has

$$
\sum_{\mathcal{J} \in \mathcal{P}_{2}(\emptyset)} \prod_{\left\{r, r^{\prime}\right\} \in \mathcal{J}} \delta_{i_{r}, i_{r^{\prime}}}=1 .
$$

The reason is that the set (of sets) $\mathcal{P}_{2}(\emptyset)$ is not the empty set (else the sum would be 0 ), but is $\{\emptyset\}$. It contains the only element $\mathcal{J}=\emptyset$ (the empty set), and the product $\prod_{\left\{r, r^{\prime}\right\} \in \mathcal{J}} \delta_{i_{r}, i_{r^{\prime}}}$ of an empty family is conventionally 1$)$.

In words, a term in (A.10) is obtained as follows. Select an even number $2 k$ of indices among the $i$ 's and also among the $j$ 's. Match the remaining $i$ 's with the remaining $j$ 's and include a factor $\delta_{i j}$ for each matched pair $(i, j)$. Divide the $2 k$ selected $i$ 's into pairs and include a factor $\delta_{i i^{\prime}}$ for each pair $\left(i, i^{\prime}\right)$. Divide the $2 k$ selected $j$ 's into pairs and include a factor $\delta_{j j^{\prime}}$ for each pair $\left(j, j^{\prime}\right)$. Different terms correspond to different such products of $\delta$ 's.

The lower rank $(n \leq 3)$ tensors write : 


$$
\begin{gathered}
\prod_{i_{1} ; j_{1}}=\delta_{i_{1} j_{1}} \\
\prod_{i_{1}, i_{2} ; j_{1}, j_{2}}=\frac{1}{2}\left(\delta_{i_{1} j_{1}} \delta_{i_{2} j_{2}}+\delta_{i_{1} j_{2}} \delta_{i_{2} j_{1}}\right)-\frac{1}{3} \delta_{i_{1} i_{2}} \delta_{j_{1} j_{2}} \\
\prod_{i_{1}, i_{2}, i_{3} ; j_{1}, j_{2}, j_{3}}=\frac{1}{6}\left(\delta_{i_{1} j_{1}} \delta_{i_{2} j_{2}} \delta_{i_{3} j_{3}}+\delta_{i_{1} j_{1}} \delta_{i_{2} j_{3}} \delta_{i_{3} j_{2}}+\delta_{i_{1} j_{2}} \delta_{i_{2} j_{1}} \delta_{i_{3} j_{3}}\right. \\
\left.+\delta_{i_{1} j_{2}} \delta_{i_{2} j_{3}} \delta_{i_{3} j_{1}}+\delta_{i_{1} j_{3}} \delta_{i_{2} j_{1}} \delta_{i_{3} j_{2}}+\delta_{i_{1} j_{3}} \delta_{i_{2} j_{2}} \delta_{i_{3} j_{1}}\right) \\
-\frac{1}{15}\left(\delta_{i_{1} i_{2}} \delta_{i_{3} j_{3}} \delta_{j_{1} j_{2}}+\delta_{i_{1} i_{2}} \delta_{i_{3} j_{2}} \delta_{j_{1} j_{3}}+\delta_{i_{1} i_{2}} \delta_{i_{3} j_{1}} \delta_{j_{2} j_{3}}\right. \\
+\delta_{i_{1} i_{3}} \delta_{i_{2} j_{3}} \delta_{j_{1} j_{2}}+\delta_{i_{1} i_{3}} \delta_{i_{2} j_{2}} \delta_{j_{1} j_{3}}+\delta_{i_{1} i_{3}} \delta_{i_{2} j_{1}} \delta_{j_{2} j_{3}} \\
\left.+\delta_{i_{2} i_{3}} \delta_{i_{1} j_{3}} \delta_{j_{1} j_{2}}+\delta_{i_{2} i_{3}} \delta_{i_{1} j_{2}} \delta_{j_{1} j_{3}}+\delta_{i_{2} i_{3}} \delta_{i_{1} j_{1}} \delta_{j_{2} j_{3}}\right)
\end{gathered}
$$

\section{Reduction to a 3-dimensional problem}

We now turn to proofs of the above results. As a preliminary step, observe that the problem reduces itself to a 3-dimensional problem. Indeed, due to Lorentz covariance, it is enough to consider a particle at rest, namely $v=(1, \overrightarrow{0})$. Then the transversity condition 3) amounts saying that $\varepsilon_{\mu_{1} \cdots \mu_{J}}=0$ if one of the indices is 0 . Therefore, a polarization tensor is completely determined by its purely spatial components $\varepsilon_{i_{1} \cdots i_{J}}$, all the other components being zero. On these 3-dimensional tensors, the conditions of symmetry 1) and of tracelessness 2) write :

$\left.1^{\prime}\right)$ Symmetry : $\varepsilon_{i_{1} \cdots i_{J}}=\varepsilon_{i_{\sigma(1)} \cdots i_{\sigma(J)}}$ for any permutation $\sigma$ of $1, \cdots, J$.

$2^{\prime}$ ) Tracelessness : $\sum_{i} \varepsilon_{i i i_{3} \cdots i_{J}}=0$ (when $J \geq 2$ ).

The orthonormality conditions (A.1) writes

$$
\sum_{i_{1} \cdots i_{J}} \varepsilon_{i_{1} \cdots i_{J}}^{(\lambda)}\left(\varepsilon_{i_{1} \cdots i_{J}}^{\left(\lambda^{\prime}\right)}\right)^{*}=\delta_{\lambda \lambda^{\prime}}
$$

The tensor $\Pi$ is also purely spatial and, according to (A.2), is given by :

$$
\prod_{i_{1} \cdots i_{J} ; i_{1}^{\prime} \cdots i_{J}^{\prime}}=\sum_{\lambda=-J}^{J} \varepsilon_{i_{1} \cdots i_{J}}^{(\lambda)}\left(\varepsilon_{i_{1}^{\prime} \cdots i_{J}^{\prime}}^{(\lambda)}\right)^{*}
$$


But the preceding consideration identifies this spatial $\prod$ as the projection operator, in the space of tensors of $\operatorname{rank} J$, on the subspace of traceless symmetric tensors. Indeed, according to (A.16), the tensor $\sum_{i_{1}^{\prime} \cdots i_{J}^{\prime}} \prod_{i_{1} \cdots i_{J} ; i_{1}^{\prime} \cdots i_{J}^{\prime}} T_{i_{1}^{\prime} \cdots i_{J}^{\prime}}$ is traceless symmetric for any tensor $T_{i_{1} \cdots i_{J}}$ and, according to (A.15) and (A.16), one has

$$
\sum_{i_{1}^{\prime} \cdots i_{J}^{\prime}} \prod_{i_{1} \cdots i_{J} ; i_{1}^{\prime} \cdots i_{J}^{\prime}} \varepsilon_{i_{1}^{\prime} \cdots i_{J}^{\prime}}=\varepsilon_{i_{1} \cdots i_{J}}
$$

for any traceless symmetric tensor $\varepsilon_{i_{1} \cdots i_{J}}$.

The problem of finding the projector on the polarization tensors is now reduced to the problem of finding the projector on the spatial symmetric traceless tensors.

\section{Deduction of the projector's particular matrix elements by standard methods of angular momentum coupling}

Let us now turn to a proof of (A.3). The space of rank $J$ tensors is just the tensor product of a number $J$ of the angular momentum 1 representation of the rotation group. The subspace of traceless symmetric tensors is just the subspace of angular momentum $J$, as can be understood since this subspace is used to describe the spin states of a particle of spin $J$.

Our problem is now reduced to the coupling of $J$ copies of the angular momentum 1 into a total angular momentum $J$. We now on use standard methods of angular momentum coupling.

The Clebsch-Gordan coefficients for the coupling of two angular momenta $J_{1}$ and $J_{2}$ to the maximum value $J_{1}+J_{2}$ has the simple following factorized form :

$$
<J_{1}+J_{2}, M \mid J_{1}, J_{2}, M_{1}, M_{2}>=\delta_{M, M_{1}+M_{2}} \frac{c\left(J_{1}, M_{1}\right) c\left(J_{2}, M_{2}\right)}{c\left(J_{1}+J_{2}, M\right)}
$$

with

$$
c(J, M)=\frac{\sqrt{(2 J) !}}{\sqrt{(J+M) !(J-M) !}} .
$$

The coupling coefficients of three angular momenta $J_{1}, J_{2}, J_{3}$ to the maximum value $J_{1}+J_{2}+J_{3}$, defined by 


$$
\begin{gathered}
<J_{1}+J_{2}+J_{3}, M \mid J_{1}, J_{2}, J_{3}, M_{1}, M_{2}, M_{3}>= \\
\sum_{M^{\prime}}<J_{1}+J_{2}+J_{3}, M\left|J_{1}+J_{2}, J_{3}, M^{\prime}, M_{3}><J_{1}+J_{2}, M^{\prime}\right| J_{1}, J_{2}, M_{1}, M_{2}>
\end{gathered}
$$

is easily calculated from (A.17) :

$$
<J_{1}+J_{2}+J_{3}, M \mid J_{1}, J_{2}, J_{3}, M_{1}, M_{2}, M_{3}>=\delta_{M, M_{1}+M_{2}+M_{3}} \frac{c\left(J_{1}, M_{1}\right) c\left(J_{2}, M_{2}\right) c\left(J_{3}, M_{3}\right)}{c\left(J_{1}+J_{2}+J_{3}, M\right)}
$$

Moreover, these coefficients do not depend on the particular order of coupling chosen in (A.19) (first coupling $J_{1}$ and $J_{2}$, and then coupling the result to $J_{3}$ ).

By a simple recursive argument, one finds from (A.19) that the coupling coefficients of $n$ angular momenta $J_{1}, \cdots, J_{n}$ to the maximum value $J_{1}+\cdots+J_{n}$ is given by

$$
<J_{1}+\cdots+J_{n}, M \mid J_{1}, \cdots, J_{n}, M_{1}, \cdots, M_{n}>=\delta_{M, M_{1}+\cdots+M_{n}} \frac{c\left(J_{1}, M_{1}\right) \cdots c\left(J_{n}, M_{n}\right)}{c\left(J_{1}+\cdots+J_{n}, M_{n}\right)}
$$

and is independent of the order of the couplings. Remind that the $\mid J_{1}+\cdots+J_{n}, M>$ are basis states of the $J_{1}+\cdots+J_{n}$ angular momentum subspace in the tensorial product of the $J_{1} \cdots J_{n}$ representation spaces of $S U(2)$, and that the coefficient $<J_{1}+\cdots+J_{n}, M \mid J_{1}, \cdots, J_{n}, M_{1}, \cdots, M_{n}>$ is the scalar product of the state $\mid J_{1}+$ $\cdots+J_{n}, M>$ with the basis state

$$
\left|J_{1}, \cdots, J_{n}, M_{1}, \cdots, M_{n}>=\right| J_{1}, M_{1}>\otimes \cdots \otimes \mid J_{n}, M_{n}>
$$

in the tensorial product space.

Now we may take the case of interest to us, $J_{1}=\cdots=J_{n}=1$, with the $J=1$ representation of $S U(2)$ in the form of the ordinary rotations in $\mathbb{C}^{3}$ space (complexified ordinary three-dimensional space). The tensorial product space is just the space of tensors of order $n$, and the $J_{1}+\cdots+J_{n}=n$ subspace is just the subspace of traceless symmetric tensors. The states $\mid 1, \cdots, 1, M_{1}, \cdots, M_{n}>$ are the tensorial products of standard basis vectors $\mid 1, M>$ of $\mathbb{C}^{3}$, and the states $\mid n, M>$ constitute a standard basis of symmetric tensors. We are interested by the scalar product of 
the tensors $\mid n, M>$ with the tensors $\vec{x}^{\otimes n}\left(\left(\vec{x}^{\otimes n}\right)_{i_{1} \cdots i_{n}}=x_{i_{1}} \cdots x_{i_{n}}\right)$ for any $\vec{x} \in \mathbb{C}^{3}$. Therefore, we have to expand the tensors $\vec{x}^{\otimes n}$ in the basis $\left|1, \cdots, 1, M_{1}, \cdots, M_{n}\right\rangle$.

The qualifier "standard" above means in conformity to the standard definition of the Clebsch-Gordan coefficients. The standard basis if $\mathbb{C}^{3}$ is :

$$
\begin{aligned}
& \mid 1,1>=\overrightarrow{f_{1}}=-\frac{1}{\sqrt{2}}\left(\vec{e}_{1}+i \vec{e}_{2}\right) \\
& \mid 1,0>=\overrightarrow{f_{0}}=\vec{e}_{3} \\
& \mid 1,-1>=\vec{f}_{-1}=\frac{1}{\sqrt{2}}\left(\vec{e}_{1}-i \vec{e}_{2}\right)
\end{aligned}
$$

where $\left(\vec{e}_{1}, \vec{e}_{2}, \vec{e}_{3}\right)$ is the Cartesian basis. Then a vector $\vec{x}=x_{1} \vec{e}_{1}+x_{2} \vec{e}_{2}+x_{3} \vec{e}_{3} \in \mathbb{C}^{3}$ writes

$$
\vec{x}=-\frac{x_{1}-i x_{2}}{\sqrt{2}} \vec{f}_{1}+\frac{x_{1}+i x_{2}}{\sqrt{2}} \vec{f}_{-1}+x_{3} \vec{f}_{0}
$$

and the tensor $(\vec{x})^{\otimes n}$ is expanded as :

$$
\begin{gathered}
(\vec{x})^{\otimes n}=\sum_{k, k^{\prime}} \frac{n !}{k ! k^{\prime} !\left(n-k-k^{\prime}\right) !}(-1)^{k}\left(\frac{x_{1}-i x_{2}}{\sqrt{2}}\right)^{k}\left(\frac{x_{1}+i x_{2}}{\sqrt{2}}\right)^{k^{\prime}}\left(x_{3}\right)^{n-k-k^{\prime}} \\
\operatorname{Sym}\left(\vec{f}_{1}\right)^{\otimes k} \otimes\left(\vec{f}_{-1}\right)^{\otimes k^{\prime}} \otimes\left(\vec{f}_{0}\right)^{\otimes n-k-k^{\prime}}
\end{gathered}
$$

where Sym is the projector on symmetric tensors

$$
(\operatorname{Sym} T)_{i_{1} \cdots i_{n}}=\frac{1}{n !} \sum_{\sigma} T_{i_{\sigma(1)} \cdots i_{\sigma(n)}} .
$$

Actually, equipped with the symmetrized product, the symmetric tensors constitute a commutative algebra, so that formula (A.26) is just obtained by multinomial expansion.

Then we have

$$
\begin{gathered}
<n, M \mid(\vec{x})^{\otimes n}>=\sum_{k, k^{\prime}} \frac{n !}{k ! k^{\prime} !\left(n-k-k^{\prime}\right) !}(-1)^{k}\left(\frac{x_{1}-i x_{2}}{\sqrt{2}}\right)^{k}\left(\frac{x_{1}+i x_{2}}{\sqrt{2}}\right)^{k^{\prime}}\left(x_{3}\right)^{n-k-k^{\prime}} \\
<n, M \mid\left(\vec{f}_{1}\right)^{\otimes k} \otimes\left(\vec{f}_{-1}\right)^{\otimes k^{\prime}} \otimes\left(\vec{f}_{0}\right)^{\otimes n-k-k^{\prime}}>
\end{gathered}
$$


where the Sym operator has been dropped because the coupling coefficients do not depend on the order of the couplings. Formula (A.22) now readily gives :

$$
\begin{gathered}
<n, M \mid(\vec{x})^{\otimes n}>=\sum_{k, k^{\prime}} \frac{n !}{k ! k^{\prime} !\left(n-k-k^{\prime}\right) !}(-1)^{k}\left(\frac{x_{1}-i x_{2}}{\sqrt{2}}\right)^{k}\left(\frac{x_{1}+i x_{2}}{\sqrt{2}}\right)^{k^{\prime}}\left(x_{3}\right)^{n-k-k^{\prime}} \\
\delta_{M, k-k^{\prime}} \frac{c(1,1)^{k} c(1,0)^{n-k-k^{\prime}} c(1,-1)^{k^{\prime}}}{c(n, M)} .
\end{gathered}
$$

An easy calculation (just undoing the multinomial expansion) gives the following generating function for these $<n, M \mid(\vec{x})^{\otimes n}>$ :

$$
\begin{gathered}
\sum_{M=-n}^{n} c(n, M)<n, M \mid(\vec{x})^{\otimes n}>s^{M}= \\
{\left[-c(1,1) \frac{x_{1}-i x_{2}}{\sqrt{2}} s+c(1,0) x_{3}-c(1,-1) \frac{x_{1}+i x_{2}}{\sqrt{2}} s^{-1}\right]^{n} .}
\end{gathered}
$$

According to formula (A.19), we have

$$
\begin{aligned}
& c(n, M)=\frac{\sqrt{(2 n) !}}{\sqrt{(n+M) !(n-M) !}} \\
& c(1,1)=1, \quad c(1,0)=\sqrt{2}, \quad c(1,-1)=1 .
\end{aligned}
$$

Therefore

$$
\begin{gathered}
\sum_{M=-n}^{n} \frac{\sqrt{(2 n) !}}{\sqrt{(n+M) !(n-M) !}}<n, M \mid(\vec{x})^{\otimes n}>s^{M}= \\
2^{n / 2}\left(-\frac{x_{1}-i x_{2}}{2} s+x_{3}+\frac{x_{1}+i x_{2}}{2} s^{-1}\right)^{n} .
\end{gathered}
$$

Comparing this to the generating function for the solid spherical harmonics $\mathcal{Y}_{L}^{M}(\vec{x})=$ $|\vec{x}|^{L} Y_{L}^{M}(\widehat{x})$, which is :

$\sum_{M=-L}^{L} \frac{(L) !}{\sqrt{(L-M) !(L+M) !}} \mathcal{Y}_{L}^{M}(\vec{r}) s^{M}=\frac{\sqrt{2 L+1}}{\sqrt{4 \pi}}\left(\frac{x_{1}-i x_{2}}{2} s^{-1}+x_{3}-\frac{x_{1}+i x_{2}}{2} s\right)^{L}$

we arrive at the fundamental result : 


$$
<n, M \mid(\vec{x})^{\otimes n}>=2^{n / 2} \frac{n !}{\sqrt{(2 n+1) !}} \sqrt{4 \pi} \mathcal{Y}_{n}^{M}(\vec{x})^{*} .
$$

From this we compute the matrix element $<(\vec{y})^{\otimes n}\left|\prod_{n}\right|(\vec{x})^{\otimes n}>$ of the seeked projector $\prod_{n}$ (on the traceless symmetric tensors). One has

$$
\begin{gathered}
<(\vec{y})^{\otimes n}\left|\prod_{n}\right|(\vec{x})^{\otimes n}>=\sum_{M=-n}^{n}<n, M\left|(\vec{y})^{\otimes n}>^{*}<n, M\right|(\vec{x})^{\otimes n}> \\
=2^{n} \frac{(n !)^{2}}{(2 n+1) !} 4 \pi \sum_{M=-n}^{n} \mathcal{Y}_{n}^{M}(\vec{y}) \mathcal{Y}_{n}^{M}(\vec{x})^{*}
\end{gathered}
$$

and using

$$
\sum_{M=-n}^{n} \mathcal{Y}_{n}^{M}(\vec{x}) \mathcal{Y}_{n}^{M}(\vec{y})^{*}=\frac{2 n+1}{4 \pi}|\vec{x}|^{n}|\vec{y}|^{n} P_{n}\left(\frac{\vec{x} \cdot \vec{y}}{|\vec{x}||\vec{y}|}\right)
$$

one readily obtains :

$$
<(\vec{y})^{\otimes n}\left|\prod_{n}\right|(\vec{x})^{\otimes n}>=2^{n} \frac{(n !)^{2}}{(2 n) !}|\vec{x}|^{n}|\vec{y}|^{n} P_{n}\left(\frac{\vec{x} \cdot \vec{y}}{|\vec{x}||\vec{y}|}\right) .
$$

One may then introduce explicit expressions for the Legendre polynomials $P_{n}$ :

$$
\begin{gathered}
P_{n}(x)=\frac{1}{2^{n}} \sum_{0 \leq k \leq n / 2}(-1)^{k} \frac{(2 n-2 k) !}{k !(n-k) !(n-2 k) !} x^{n-2 k} \\
P_{n}(x)=\sum_{0 \leq k \leq n / 2}(-1)^{k} \frac{1}{2^{2 k}} \frac{n !}{(k !)^{2}(n-2 k) !} x^{n-2 k}\left(1-x^{2}\right)^{k}
\end{gathered}
$$

and we obtain the following explicit expressions for $\left\langle(\vec{y})^{\otimes n}\left|\prod_{n}\right|(\vec{x})^{\otimes n}>\right.$ :

$$
\begin{aligned}
<(\vec{y})^{\otimes n}\left|\prod_{n}\right|(\vec{x})^{\otimes n}> & =\sum_{0 \leq k \leq n / 2} C_{n, k}\left(\vec{x}^{2}\right)^{k}(\vec{x} \cdot \vec{y})^{n-2 k}\left(\vec{y}^{2}\right)^{k} \\
& =\sum_{0 \leq k \leq n / 2} C_{n, k}^{\prime}(\vec{x} \cdot \vec{y})^{n-2 k}\left[\vec{x}^{2} \vec{y}^{2}-(\vec{x} \cdot \vec{y})^{2}\right]^{k} \\
C_{n, k} & =(-1)^{k} \frac{(n !)^{2}}{(2 n) !} \frac{(2 n-2 k) !}{k !(n-k) !(n-2 k) !} \\
C_{n, k}^{\prime} & =(-1)^{k} 2^{n-2 k} \frac{(n !)^{2}}{(2 n) !} \frac{n !}{(k !)^{2}(n-2 k) !} .
\end{aligned}
$$


To go back to an arbitrary velocity $v$ and obtain (A.3), just set $\vec{x}=\vec{v}_{i}, \vec{y}=\vec{v}_{f}$ and use the following formulae :

$$
\begin{aligned}
& \vec{v}_{i}^{2}=\left(v_{i} \cdot v\right)^{2}-v_{i}^{2}=w_{i}^{2}-1 \\
& \vec{v}_{f}^{2}=\left(v_{f} \cdot v\right)^{2}-v_{f}^{2}=w_{f}^{2}-1 \\
& \vec{v}_{i} \cdot \vec{v}_{f}=\left(v_{i} \cdot v\right)\left(v_{f} \cdot v\right)-v_{i} \cdot v_{f}=w_{i} w_{f}-w_{i f}
\end{aligned}
$$

\section{Deduction of the projector itself from its particular matrix elements}

We now present a deduction of $\prod_{i_{1}, \cdots, i_{n} ; j_{1}, \cdots, j_{n}}$ from the matrix elements $<(\vec{y})^{\otimes n}\left|\prod_{n}\right|(\vec{x})^{\otimes n}>$. To see how to proceed, let us consider a multilinear function $F\left(\vec{x}_{1}, \cdots, \vec{x}_{n}\right)$, which is symmetric in the permutations of its $n$ vector variables $\vec{x}_{1}, \cdots, \vec{x}_{n}$. Then it can be recovered from its diagonal values $F\left((\vec{x})_{n}\right)=F(\vec{x}, \cdots, \vec{x})$ by the following formula :

$$
F\left(\vec{x}_{1}, \cdots, \vec{x}_{n}\right)=\frac{(-1)^{n}}{n !} \sum_{\substack{s_{1}, \cdots, s_{n} \\ 0 \leq s_{i} \leq 1}}(-1)^{s_{1}+\cdots+s_{n}} F\left(\left(s_{1} \vec{x}_{1}+\cdots+s_{n} \vec{x}_{n}\right)_{n}\right) .
$$

Indeed, expanding $F\left(\left(s_{1} \vec{x}_{1}+\cdots+s_{n} \vec{x}_{n}\right)_{n}\right)$ by multinearity and collecting terms equal by symmetry, one has :

$$
F\left(\left(s_{1} \vec{x}_{1}+\cdots+s_{n} \vec{x}_{n}\right)_{n}\right)=\sum_{\substack{q_{1}, \cdots, q_{n} \\ q_{1}+\cdots+q_{n}=n}} \frac{n !}{q_{1} ! \cdots q_{n} !} s_{1}^{q_{1}} \cdots s_{n}^{q_{n}} F\left(\left(\vec{x}_{1}\right)_{q_{1}}, \cdots,\left(\vec{x}_{n}\right)_{q_{n}}\right)
$$

where the notation $\left(\vec{x}_{i}\right)_{q_{i}}$ (also used in (A.42)) stands for the $q_{i}$-uple $\left(\vec{x}_{i}, \cdots, \vec{x}_{i}\right)$. A term in (A.43) with some $q_{i}$ vanishing gives no contribution to (A.42) because it does not depend on $s_{i}$, and the corresponding $s_{i}=0$ and $s_{i}=1$ terms in (A.42) cancels. Then, since $q_{1}+\cdots+q_{n}=n$, the only term of (A.43) contributing to (A.42) is $q_{1}=\cdots=q_{n}=1$. The right-hand side of (A.42) is therefore equal to :

$$
\frac{(-1)^{n}}{n !} \sum_{\substack{s_{1}, \cdots, s_{n} \\ 0 \leq s_{i} \leq 1}}(-1)^{s_{1}+\cdots+s_{n}} n ! s_{1} \cdots s_{n} F\left(\vec{x}_{1}, \cdots, \vec{x}_{n}\right)=F\left(\vec{x}_{1}, \cdots, \vec{x}_{n}\right) .
$$


Using (A.42), we can now deduce $\prod_{i_{1}, \cdots, i_{n} ; j_{1}, \cdots, j_{n}}$ from the matrix elements in two steps. As a first step, let us apply formula (A.42) to the multilinear symmetric function

$$
\left(\vec{y}_{1}, \cdots, \vec{y}_{n}\right) \rightarrow<\vec{y}_{1} \otimes \cdots \otimes \vec{y}_{n}\left|\prod_{n}\right|(\vec{x})^{\otimes n}>
$$

with $\vec{x}$ fixed. This gives :

$$
\left\langle\vec{y}_{1} \otimes \cdots \otimes \vec{y}_{n}\left|\prod_{n}\right|(\vec{x})^{\otimes n}\right\rangle=\frac{(-1)^{n}}{n !} \sum_{\substack{s_{1}, \cdots, s_{n} \\ 0 \leq s_{i} \leq 1}}(-1)^{s_{1}+\cdots+s_{n}}\left\langle\left(\sum_{i} s_{i} \vec{y}_{i}\right)^{\otimes n}\left|\prod_{n}\right|(\vec{x})^{\otimes n}\right\rangle
$$

or, using (A.39)

$$
\begin{gathered}
<\vec{y}_{1} \otimes \cdots \otimes \vec{y}_{n}\left|\prod_{n}\right|(\vec{x})^{\otimes n}>=\frac{(-1)^{n}}{n !} \sum_{0 \leq k \leq n / 2} C_{n, k}\left(\vec{x}^{2}\right)^{k} \\
\sum_{\substack{s_{1}, \cdots, s_{n} \\
0 \leq s_{i} \leq 1}}(-1)^{s_{1}+\cdots+s_{n}}\left(\sum_{i} s_{i}\left(\vec{y}_{i} \cdot \vec{x}\right)\right)^{n-2 k}\left(\left(\sum_{i} s_{i} \vec{y}_{i}\right)^{2}\right)^{k} .
\end{gathered}
$$

Then we work out multinomial expansions :

$$
\begin{gathered}
\left(\sum_{i} s_{i}\left(\vec{y}_{i} \cdot \vec{x}\right)\right)^{n-2 k}=\sum_{\substack{u_{1}, \cdots, u_{n} \geq 0 \\
u_{1}+\cdots+u_{n}=n-2 k}} \frac{(n-2 k) !}{u_{1} ! \cdots u_{n} !} \prod_{i=1}^{n}\left(s_{i}\right)^{u_{i}}\left(\vec{y}_{i} \cdot \vec{x}\right)^{u_{i}} \\
\left(\left(\sum_{i} s_{i} \vec{y}_{i}\right)^{2}\right)^{k}=\left(\sum_{1 \leq i, i^{\prime} \leq n} s_{i} s_{i^{\prime}}\left(\vec{y}_{i} \cdot \vec{y}_{i^{\prime}}\right)\right)^{k} \\
=\sum_{\substack{v_{11}, v_{12}, \cdots, v_{n-1, n}, v_{n n \geq 0} \geq 0 \\
v_{11}+v_{12}+\cdots+v_{n n}=k}}^{n} \frac{k !}{v_{11} ! v_{12} ! \cdots v_{n n} !} \prod_{i, i^{\prime}=1}^{n}\left(s_{i} s_{i^{\prime}}\right)^{v_{i i^{\prime}}}\left(\vec{y}_{i} \cdot \vec{y}_{i^{\prime}}\right)^{v_{i i^{\prime}}} .
\end{gathered}
$$

Using this in (A.46) and collecting the powers of the $s_{i}$, we have :

$$
\begin{aligned}
& <\vec{y}_{1} \otimes \cdots \otimes \vec{y}_{n}\left|\prod_{n}\right|(\vec{x})^{\otimes n}>=\frac{(-1)^{n}}{n !} \sum_{0 \leq k \leq n / 2} C_{n, k}\left(\vec{x}^{2}\right)^{k} \\
& \sum_{\substack{u_{1}, \cdots, u_{n} \geq 0 \\
u_{1}+\cdots+u_{n}=n-2 k}} \sum_{\substack{v_{11}, v_{12}, \cdots, v_{n-1, n}, v_{n n \geq 0} \geq 0 \\
v_{11}+v_{12}+\cdots+v_{n n}=k}} \frac{(n-2 k) !}{u_{1} ! \cdots u_{n} !} \frac{k !}{v_{11} ! v_{12} ! \cdots v_{n n} !} \\
& \sum_{\substack{s_{1}, \cdots, s_{n} \\
0 \leq s_{i} \leq 1}}(-1)^{s_{1}+\cdots+s_{n}}\left[\prod_{i=1}^{n}\left(s_{i}\right)^{p_{i}}\right]\left[\prod_{i=1}^{n}\left(\vec{y}_{i} \cdot \vec{x}\right)^{u_{i}}\right]\left[\prod_{i, i^{\prime}=1}^{n}\left(\vec{y}_{i} \cdot \vec{y}_{i^{\prime}}\right)^{v_{i i^{\prime}}}\right]
\end{aligned}
$$


where the exponent $p_{i}$ of $s_{i}$ is :

$$
p_{i}=u_{i}+\sum_{i^{\prime}=1}^{n}\left(v_{i^{\prime} i}+v_{i i^{\prime}}\right) .
$$

Notice now that, for values of the $u_{i}$ 's and of the $v_{i i^{\prime}}$ 's such that some exponent $p_{i_{0}}$ vanishes, the $s_{i_{0}}=0$ and the $s_{i_{0}}=1$ terms cancel. Since, according to the constraints on the $u_{i}$ 's and the $v_{i i^{\prime}}$ 's, one has

$$
\sum_{i=1}^{n} p_{i}=\sum_{i=1}^{n} u_{i}+2 \sum_{i, i^{\prime}=1}^{n} v_{i i^{\prime}}=n,
$$

we are left with the values of the $u_{i}$ 's and of the $v_{i i^{\prime}}$ 's such that $p_{1}=\cdots=p_{n}=1$. These values can then be only 0 or 1 , so that $u_{i} !=1$ and $v_{i i^{\prime}} !=1$. Moreover we have then a factor $s_{1} \cdots s_{n}$, so that only $s_{1}=\cdots=s_{n}=1$ contributes. So, (A.49) reduces to :

$$
\begin{aligned}
<\vec{y}_{1} \otimes \cdots \otimes \vec{y}_{n}\left|\prod_{n}\right|(\vec{x})^{\otimes n}>= & \sum_{0 \leq k \leq n / 2} \frac{k !(n-2 k) !}{n !} C_{n, k}\left(\vec{x}^{2}\right)^{k} \\
\sum_{\substack{u_{1}, \cdots, u_{n}, v_{11}, v_{11}, v_{12}, \cdots, v_{n-1, n}, v_{n n} \geq 0 \\
u_{1}+\cdots+u_{n}=n-2 k \\
u_{i}+v_{1 i}+\cdots+v_{n i}+v_{i 1}+\cdots+v_{i n}=1}} & {\left[\prod_{i=1}^{n}\left(\vec{y}_{i} \cdot \vec{x}\right)^{u_{i}}\right]\left[\prod_{\substack{i, i^{\prime}=1 \\
i}}^{n}\left(\vec{y}_{i} \cdot \vec{y}_{i^{\prime}}\right)^{v_{i i^{\prime}}}\right] }
\end{aligned}
$$

where we have dropped the constraint $\sum_{i, i^{\prime}=1}^{n} v_{i i^{\prime}}=k$ since it is implied by the remaining constraints.

Since the $u_{i}$ 's take only the values 0 or 1 , we can replace $\left(u_{1}, \cdots, u_{n}\right)$, as summation variable, by subsets $I$ of $\{1, \cdots, n\}$. It will be convenient to use the subset related to $\left(u_{1}, \cdots, u_{n}\right)$ by $I=\left\{i \mid u_{i}=0\right\}$. The constraint $u_{1}+\cdots+u_{n}=n-2 k$ is translated into the constraint $|I|=2 k$, and formula (A.52) becomes :

$$
\begin{gathered}
<\vec{y}_{1} \otimes \cdots \otimes \vec{y}_{n}\left|\prod_{n}\right|(\vec{x})^{\otimes n}>=\sum_{0 \leq k \leq n / 2} \frac{k !(n-2 k) !}{n !} C_{n, k} \\
\left(\vec{x}^{2}\right)^{k} \sum_{\substack{I \subset\{1 \cdots n\} \\
|I|=2 k}}\left[\prod_{i \notin I}\left(\vec{y}_{i} \cdot \vec{x}\right)\right] \sum_{\substack{v_{11}, v_{11}, v_{12}, \cdots, v_{n-1, n}, v_{n n} \geq 0 \\
v_{1 i}+\cdots v_{n i}+v_{i 1}+\cdots+v_{i n}=0(i \notin I) \\
v_{1 i}+\cdots+v_{n i}+v_{i 1}+\cdots+v_{i n}=1(i \in I)}}\left[\prod_{i, i^{\prime} \in I}\left(\vec{y}_{i} \cdot \vec{y}_{i^{\prime}}\right)^{v_{i i^{\prime}}}\right] .
\end{gathered}
$$

Consider now the constraints on the $v_{i i^{\prime}}$ 's. If $i \notin I$ or $i^{\prime} \notin I$, we have $v_{i i^{\prime}}=0$. We are left with the $v_{i i^{\prime}}$ for $i, i^{\prime} \in I$, constrained by : 


$$
\sum_{i^{\prime} \in I}\left(v_{i i^{\prime}}+v_{i^{\prime} i}\right)=1 \quad(\text { for any } i \in I) .
$$

Notice that $v_{i i}=0$, since $v_{i i}$ occurs twice in this sum. Let us then replace the $v_{i i^{\prime}}$ 's, as summation variable, by the set $\mathcal{J}$ of two-element subsets of $I$ related to the $v_{i i}$ 's by

$$
\mathcal{J}=\left\{\left\{i, i^{\prime}\right\} \mid v_{i i^{\prime}}+v_{i^{\prime} i}=1\right)
$$

For any $i \in I$, there is, according to (A.54), one and only one $i^{\prime} \in I$ such that $\left\{i, i^{\prime}\right\} \in \mathcal{J}$. In other words, $\mathcal{J}$ belongs to the set $\mathcal{P}_{2}(I)$ of partitions of $I$ by twoelement subsets. Conversely, if $\mathcal{J} \in \mathcal{P}_{2}(I)$, the values of $v_{i i^{\prime}}+v_{i^{\prime} i}$ defined by (A.55), namely

$$
v_{i i^{\prime}}+v_{i^{\prime} i}=1 \text { if }\left\{i, i^{\prime}\right\} \in \mathcal{J}, \quad v_{i i^{\prime}}+v_{i^{\prime} i}=0 \text { if }\left\{i, i^{\prime}\right\} \notin \mathcal{J}
$$

do satisfy the constraints (A.54), since for any $i \in I$, there is one and only one $i^{\prime} \in I$ such that $\left\{i, i^{\prime}\right\} \in \mathcal{J}$. Now, (A.56) does not determine completely the values of the $v_{i i^{\prime}}$ 's. When $\left\{i, i^{\prime}\right\} \notin \mathcal{J}$ we must have $v_{i i^{\prime}}=v_{i^{\prime} i}=0$, but when $\left\{i, i^{\prime}\right\} \in \mathcal{J}$ we have two solutions : $v_{i i^{\prime}}=1, v_{i^{\prime} i}=0$ and $v_{i i^{\prime}}=0, v_{i^{\prime} i}=1$. Since $|\mathcal{J}|=k$, we have in all $2^{k}$ values of the $v_{i i^{\prime}}$ 's corresponding to each $\mathcal{J} \in \mathcal{P}_{2}(I)$. However, values of the $v_{i i^{\prime}}$ 's corresponding to a same $\mathcal{J}$ give equal terms in (A.53) and, lumping these terms together, formula (A.53) becomes :

$$
\begin{gathered}
<\vec{y}_{1} \otimes \cdots \otimes \vec{y}_{n}\left|\prod_{n}\right|(\vec{x})^{\otimes n}>=\sum_{0 \leq k \leq n / 2} 2^{k} \frac{k !(n-2 k) !}{n !} C_{n, k} \\
\left(\vec{x}^{2}\right)^{k} \sum_{\substack{I \subset\{1 \cdots n\} \\
|I|=2 k}}\left[\prod_{i \notin I}\left(\vec{y}_{i} \cdot \vec{x}\right)\right]\left[\sum_{\mathcal{J} \in \mathcal{P}_{2}(I)} \prod_{\left\{i, i^{\prime}\right\} \in \mathcal{J}}\left(\vec{y}_{i} \cdot \vec{y}_{i^{\prime}}\right)\right] .
\end{gathered}
$$

As a second (and last) step, let us apply formula (A.42) to the multilinear symmetric function

$$
\left(\vec{x}_{1}, \cdots, \vec{x}_{n}\right) \rightarrow<\vec{y}_{1} \otimes \cdots \otimes \vec{y}_{n}\left|\prod_{n}\right| \vec{x}_{1} \otimes \cdots \otimes \vec{x}_{n}>
$$

with $\left(\vec{y}_{1}, \cdots, \vec{y}_{n}\right)$ fixed. This gives : 


$$
\begin{gathered}
<\vec{y}_{1} \otimes \cdots \otimes \vec{y}_{n}\left|\prod_{n}\right| \vec{x}_{1} \otimes \cdots \otimes \vec{x}_{n}>= \\
\frac{(-1)^{n}}{n !} \sum_{\substack{s_{1}, \cdots, s_{n} \\
0 \leq s_{i} \leq 1}}(-1)^{s_{1}+\cdots+s_{n}}<\vec{y}_{1} \otimes \cdots \otimes \vec{y}_{n}\left|\prod_{n}\right|\left(\sum_{j} s_{j} \vec{x}_{j}\right)^{\otimes n}>
\end{gathered}
$$

or, using (A.57)

$$
\begin{gathered}
<\vec{y}_{1} \otimes \cdots \otimes \vec{y}_{n}\left|\prod_{n}\right| \vec{x}_{1} \otimes \cdots \otimes \vec{x}_{n}>=\frac{(-1)^{n}}{n !} \sum_{0 \leq k \leq n / 2} 2^{k} \frac{k !(n-2 k) !}{n !} C_{n, k} \\
\sum_{\substack{I \subset\{1, \cdots n\} \\
|I|=2 k}}\left[\sum_{\mathcal{J} \in \mathcal{P}_{2}(I)\left\{i, i^{\prime}\right\} \in \mathcal{J}}\left(\vec{y}_{i} \cdot \vec{y}_{i^{\prime}}\right)\right] \\
\sum_{\substack{s_{1}, \cdots, s_{n} \\
0 \leq s_{j} \leq 1}}(-1)^{s_{1}+\cdots+s_{n}}\left(\left(\sum_{j} s_{j} \vec{x}_{j}\right)^{2}\right)^{k}\left[\prod_{i \notin I}\left(\sum_{j} s_{j}\left(\vec{y}_{i} \cdot \vec{x}_{j}\right)\right)\right] .
\end{gathered}
$$

Using the expansions

$$
\begin{gathered}
\prod_{i \notin I}\left(\sum_{j} s_{j}\left(\vec{y}_{i} \cdot \vec{x}_{j}\right)\right)=\sum_{\substack{u_{i j} \geq 0(i \notin I, 1 \leq j \leq n) \\
u_{i 1}+\cdots+u_{i n}=1}}\left[\prod_{\substack{i \notin I \\
1 \leq j \leq n}}\left(s_{j}\right)^{u_{i j}}\left(\vec{y}_{i} \cdot \vec{x}_{j}\right)^{u_{i j}}\right] \\
\left(\left(\sum_{j} s_{j} \vec{x}_{j}\right)^{2}\right)^{k}=\left(\sum_{1 \leq j, j^{\prime} \leq n} s_{j} s_{j^{\prime}}\left(\vec{x}_{j} \cdot \vec{x}_{j^{\prime}}\right)\right)^{k} \\
=\sum_{\substack{v_{11}, v_{12}, \cdots, v_{n-1, n}, v_{n n} \geq 0 \\
v_{11}+v_{12}+\cdots+v_{n n}=k}} \frac{k !}{v_{11} ! v_{12} ! \cdots v_{n n} !}\left[\prod_{j, j^{\prime}=1}^{n}\left(s_{j} s_{j^{\prime}}\right)^{\left.v_{j^{\prime}}\left(\vec{x}_{j} \cdot \vec{x}_{j^{\prime}}\right)^{v_{j j^{\prime}}}\right]}\right.
\end{gathered}
$$

in (A.60) and collecting the powers of the $s_{i}$, we have :

$$
\begin{gathered}
<\vec{y}_{1} \otimes \cdots \otimes \vec{y}_{n}\left|\prod_{n}\right| \vec{x}_{1} \otimes \cdots \otimes \vec{x}_{n}>=\frac{(-1)^{n}}{n !} \sum_{0 \leq k \leq n / 2} 2^{k} \frac{k !(n-2 k) !}{n !} C_{n, k} \\
\sum_{\substack{I \subset\{1, \ldots n\} \\
|I|=2 k}}\left[\sum_{\mathcal{J} \in \mathcal{P}_{2}(I)} \prod_{\left\{i, i^{\prime}\right\} \in \mathcal{J}}\left(\vec{y}_{i} \cdot \vec{y}_{i^{\prime}}\right)\right] \\
\sum_{\substack{u_{i j} \geq 0(i \notin I, 1 \leq j \leq n) \\
u_{i 1}+\cdots, u_{i n}=1}} \sum_{\substack{v_{11}, v_{12}, \cdots, v_{n}, 1, n, v_{n n} \geq 0 \\
v_{11}+v_{12}+\cdots, v_{n n}=k}} \frac{k !}{v_{11} ! v_{12} ! \cdots v_{n n} !}
\end{gathered}
$$




$$
\sum_{\substack{s_{1}, \cdots, s_{n} \\ 0 \leq s_{j} \leq 1}}(-1)^{s_{1}+\cdots+s_{n}}\left[\prod_{j=1}^{n}\left(s_{j}\right)^{p_{j}}\right]\left[\prod_{\substack{i \notin I \\ 1 \leq j \leq n}}\left(\vec{y}_{i} \cdot \vec{x}_{j}\right)^{u_{i j}}\right]\left[\prod_{j, j^{\prime}=1}^{n}\left(\vec{x}_{j} \cdot \vec{x}_{j^{\prime}}\right)^{v_{j j^{\prime}}}\right]
$$

where the exponent $p_{j}$ of $s_{j}$ is :

$$
p_{j}=\sum_{i \notin I} u_{i j}+\sum_{j^{\prime}=1}^{n}\left(v_{j^{\prime} j}+v_{j j^{\prime}}\right) .
$$

According to the constraints on the $u_{i j}$ 's and the $v_{j j^{\prime}}$ 's, one has

$$
\sum_{j=1}^{n} p_{j}=\sum_{\substack{i \notin 1 \\ 1 \leq j \leq n}} u_{i j}+2 \sum_{j, j^{\prime}=1}^{n} v_{j j^{\prime}}=n
$$

and, by the same arguments following eq. (A.49), one sees that only the terms $p_{1}=\cdots=p_{n}=1, s_{1}=\cdots=s_{n}=1$ contributes to (A.63). So, (A.63) reduces to :

$$
\begin{aligned}
& <\vec{y}_{1} \otimes \cdots \otimes \vec{y}_{n}\left|\prod_{n}\right| \vec{x}_{1} \otimes \cdots \otimes \vec{x}_{n}>=\sum_{0 \leq k \leq n / 2} 2^{k} \frac{(k !)^{2}(n-2 k) !}{(n !)^{2}} C_{n, k} \\
& \sum_{\substack{I \subset\{1 \cdots n\} \\
|I|=2 k}}\left[\sum_{\mathcal{J} \in \mathcal{P}_{2}(I)} \prod_{\left\{i, i^{\prime}\right\} \in \mathcal{J}}\left(\vec{y}_{i} \cdot \vec{y}_{i^{\prime}}\right)\right] \\
& \sum_{\substack{u_{i j}, v j_{j j^{\prime}} \geq 0\left(i \notin I, 1 \leq j, j^{\prime} \leq n\right) \\
\sum_{1 \leq j \leq n}^{u_{i j}=1(i \notin I)}}}\left[\prod_{\substack{i \notin I \\
1 \leq j \leq n}}\left(\vec{y}_{i} \cdot \vec{x}_{j}\right)^{u_{i j}}\right]\left[\prod_{j, j^{\prime}=1}^{n}\left(\vec{x}_{j} \cdot \vec{x}_{j^{\prime}}\right)^{v_{j j^{\prime}}}\right] .
\end{aligned}
$$

Consider the constraints on the $u_{i j}$ 's :

$$
\sum_{j=1}^{n} u_{i j}=1(\text { for all } i \notin I), \quad \sum_{i \not \mid} u_{i j} \leq 1(\text { for all } 1 \leq j \leq n)
$$

The solutions of (A.67) are in one to one correspondence with the set of injective maps $v: \mathbf{C} I \rightarrow\{1, \cdots, n\}$ by :

$$
u_{i j}=1 \text { if } j=v(i), \quad u_{i j}=0 \text { if } j \neq v(i)
$$

Indeed, the first constraint (A.67) says that to each $i \notin I$ corrresponds one and only one $j=v(i)$ such that $u_{i j}=1$, and this defines a map $v: \mathbf{C} I \rightarrow\{1, \cdots, n\}$. The 
second one says that, for each $j$, there is at most one $i \notin I$ such that $u_{i j}=1$, and this means that the map $v$ is injective.

We can therefore replace the $u_{i j}$ 's, as summation variable, by the injective maps $v: \mathbf{C} I \rightarrow\{1, \cdots, n\}$. It will be furthermore convenient to replace $v$ by the pair $(J, \sigma)$ where $J=\mathbf{C} v(\mathbf{C} I)$ is the complementary subset of the image of $v$, and $\sigma: \mathbf{C} I \rightarrow \mathbf{C} J$ is the bijective map induced by $v$. Then formula (A.68) becomes :

$$
\begin{gathered}
<\vec{y}_{1} \otimes \cdots \otimes \vec{y}_{n}\left|\prod_{n}\right| \vec{x}_{1} \otimes \cdots \otimes \vec{x}_{n}>=\sum_{0 \leq k \leq n / 2} 2^{k} \frac{(k !)^{2}(n-2 k) !}{(n !)^{2}} C_{n, k} \\
\sum_{\substack{I, J \subset\{1 \cdots n\} \\
|I|=|J|=2 k}}\left[\sum_{\mathcal{J} \in \mathcal{P}_{2}(I)} \prod_{\left\{i, i^{\prime}\right\} \in \mathcal{J}}\left(\vec{y}_{i} \cdot \vec{y}_{i^{\prime}}\right)\right]\left[\sum_{\sigma \in \mathcal{B}(C I, C J)} \prod_{i \notin I}\left(\vec{y}_{i} \cdot \vec{x}_{\sigma(i)}\right)\right] \\
\sum_{\substack{v_{j j^{\prime}} \geq 0\left(1 \leq j, j^{\prime} \leq n\right) \\
\sum_{1 \leq j^{\prime} \leq n}\left(v_{j j^{\prime}}+v_{j^{\prime} j}\right)=0(j \notin J)}}\left[\prod_{j, j^{\prime}=1}^{n}\left(\vec{x}_{j} \cdot \vec{x}_{j^{\prime}}\right)^{v_{j j^{\prime}}}\right] .
\end{gathered}
$$

This last summation on the $v_{j j^{\prime}}$ 's is exactly the same as the summation on the $v_{i i^{\prime}}$ 's in formula (A.53), and is treated in the same way. It is replaced by a summation on the partitions $\mathcal{J}^{\prime} \in \mathcal{P}_{2}(J)$ of $J$ by two-element subsets. To each $\mathcal{J}^{\prime}$ corresponds $2^{k}$ solutions of the $v_{j j^{\prime}}$ 's constraints, which give equal terms in (A.69). Finally we obtain

$$
\begin{gathered}
<\vec{y}_{1} \otimes \cdots \otimes \vec{y}_{n}\left|\prod_{n}\right| \vec{x}_{1} \otimes \cdots \otimes \vec{x}_{n}>=\sum_{0 \leq k \leq n / 2} 2^{k} \frac{(k !)^{2}(n-2 k) !}{(n !)^{2}} C_{n, k} \sum_{\substack{\begin{subarray}{c}{1, J \subset\{1 \ldots n\} \\
|I|=|J|=2 k} }} \\
{|I|}\end{subarray}}(\mathrm{A} . \\
{\left[\sum_{\mathcal{J} \in \mathcal{P}_{2}(I)} \prod_{\left\{i, i^{\prime}\right\} \in \mathcal{J}}\left(\vec{y}_{i} \cdot \vec{y}_{i^{\prime}}\right)\right]\left[\sum_{\sigma \in \mathcal{B}(C I, C J)} \prod_{i \notin I}\left(\vec{y}_{i} \cdot \vec{x}_{\sigma(i)}\right)\right]\left[\sum_{\mathcal{J}^{\prime} \in \mathcal{P}_{2}(I)} \prod_{\left\{j, j^{\prime}\right\} \in \mathcal{J}^{\prime}}\left(\vec{x}_{j} \cdot \vec{x}_{j^{\prime}}\right)\right] .}
\end{gathered}
$$

Formula (A.10) is just formula (A.70) with

$$
\left(\vec{y}_{1}, \cdots, \vec{y}_{n}\right)=\left(\vec{e}_{i_{1}}, \cdots, \vec{e}_{i_{n}}\right), \quad\left(\vec{x}_{1}, \cdots, \vec{x}_{n}\right)=\left(\vec{e}_{j_{1}}, \cdots, \vec{e}_{j_{n}}\right)
$$

where $\left(\vec{e}_{1}, \vec{e}_{2}, \vec{e}_{3}\right)$ is the Cartesian basis of $I^{3}$. 


\section{Direct proof of the expression of the projector}

The expression (A.10) for the projector $\prod_{i_{1}, \cdots, i_{n} ; j_{1}, \cdots, j_{n}}$ on the symmetric traceless tensors has been obtained by rather lengthy and indirect arguments. However, once expression (A.10) is known, it becomes possible to verify directly that it gives the seeked projector. We do this now, obtaining a new proof of (A.10). This new proof goes on, without added complications, for an arbitrary dimension $D$ of space.

We thus consider now (A.10) as a tentative formula, with unknown coefficients $f_{n, k}$. Let us enumerate the conditions for the $\prod_{i_{1}, \cdots, i_{n} ; j_{1}, \cdots, j_{n}}$ to be the components of the projector $\prod_{n}$ on the subspace of the symmetric traceless tensors (in the space of all $n$-rank tensors).

First, the image by $\prod_{n}$ of any tensor must be in the projection subspace. For the components, this means that, for fixed $\left(j_{1}, \cdots, j_{n}\right)$, the $n$-rank tensor $\prod_{i_{1}, \cdots, i_{n} ; j_{1}, \cdots, j_{n}}$ is symmetric traceless. Therefore we have the two conditions :

1) $\prod_{i_{1}, \cdots, i_{n} ; j_{1}, \cdots, j_{n}}$ is symmetric with respect to the $i$ 's.

2) $\sum_{i_{1}, i_{2}=1}^{D} \delta_{i_{1} i_{2}} \prod_{i_{1}, \cdots, i_{n} ; j_{1}, \cdots, j_{n}}=0 \quad$ (for all $\left.i_{3}, \cdots, i_{n}, j_{1}, \cdots, j_{n}\right)$.

Next, $\prod_{n}$ must transform into itself any tensor in the projection subspace. This gives the following third condition :

3) $\sum_{j_{1}, \cdots, j_{n}=1}^{D} \prod_{i_{1}, \cdots, i_{n} ; j_{1}, \cdots, j_{n}} \varepsilon_{j_{1}, \cdots, j_{n}}=\varepsilon_{i_{1}, \cdots, i_{n}}$

for any symmetric traceless tensor $\varepsilon_{i_{1}, \cdots, i_{n}}$.

Conditions $1,2,3)$ says that $\prod_{n}$ is some projector on the symmetric traceless tensors. To specify it completely, we must add that $\prod_{n}$ is the orthogonal projector (i.e. it annihilates any tensor orthogonal to all symmetric traceless tensors). It is equivalent saying that $\prod_{n}$ is a symmetric (or hermitian) operator. This gives a fourth and last condition :

4) $\prod_{i_{1}, \cdots, i_{n} ; j_{1}, \cdots, j_{n}}=\prod_{j_{1}, \cdots, j_{n} ; i_{1}, \cdots, i_{n}}$

Let us see now if we can fulfill these conditions with formula (A.10).

The conditions 1) and 4) are easy. They are satisfied independently of the coefficients $f_{n, k}$. The symmetry with respect to the $i$ 's stems from the fact that only the set $\{1, \cdots, n\}$ of the numbers which specify the $i$ 's enters in (A.10). The symmetry with respect to the exchange of the $i$ 's and $j$ 's is also clearly satisfied by (A.10). 
Condition 3) is not difficult to deal with. Notice that any term in (A.10) containing a factor $\delta_{j_{t} j_{t^{\prime}}}$ gives no contribution to the left-hand side of (A.72). This is due to the fact that $\varepsilon_{i_{1}, \cdots, i_{n}}$ is symmetric traceless, so that

$$
\sum_{j_{t}, j_{t^{\prime}}=1}^{D} \delta_{j_{t} j_{t^{\prime}}} \varepsilon_{j_{1}, \cdots, j_{n}}=0
$$

The only terms in (A.10) without any factor $\delta_{j_{t} j_{t^{\prime}}}$ are the ones with $J$ empty. But $J=\emptyset$ implies $k=0$ and $I=\emptyset$. So, with the trial formula (A.10), condition 3) takes the form :

$$
f_{n, 0} \sum_{j_{1}, \cdots, j_{n}=1}^{D} \sum_{\sigma \in \mathcal{P}_{n}} \prod_{s=1}^{n} \delta_{i_{s} j_{\sigma(s)}} \varepsilon_{j_{1}, \cdots, j_{n}}=\varepsilon_{i_{1}, \cdots, i_{n}}
$$

or, summing the $j_{i}$ 's,

$$
f_{n, 0} \sum_{\sigma \in \mathcal{P}_{n}} \varepsilon_{i_{\sigma(1)}, \cdots, i_{\sigma(n)}}=\varepsilon_{i_{1}, \cdots, i_{n}}
$$

where $\mathcal{P}_{n}$ is the set of permutations of the set $\{1, \cdots, n\}$. Taking into account the symmetry of $\varepsilon_{i_{1}, \cdots, i_{n}}$, and since $\left|\mathcal{P}_{n}\right|=n$ !, this is the same as :

$$
n ! f_{n, 0} \varepsilon_{i_{1}, \cdots, i_{n}}=\varepsilon_{i_{1}, \cdots, i_{n}} .
$$

Therefore, condition 3) just fixes $f_{n, k}$ for $k=0$ :

$$
f_{n, 0}=\frac{1}{n !}
$$

Condition 2) is the hard one. The first thing to be done is to rewrite (A.10) in a form where the occurrences of the indices $i_{1}$ and $i_{2}$ are explicit. The result of this step is formula (A.83) below. To alleviate the formulae, let us introduce the following notations :

$$
\begin{aligned}
X(I) & =\sum_{\mathcal{J} \in \mathcal{P}_{2}(I)} \prod_{\left\{r, r^{\prime}\right\} \in \mathcal{J}} \delta_{i_{r}, i_{r^{\prime}}} \\
Y(J) & =\sum_{\mathcal{J}^{\prime} \in \mathcal{P}_{2}(J)} \prod_{\left\{t, t^{\prime}\right\} \in \mathcal{J}^{\prime}} \delta_{j_{t}, j_{t^{\prime}}}
\end{aligned}
$$




$$
Z(I, J)=\sum_{\sigma \in \mathcal{B}(I, J)} \prod_{s \in I} \delta_{i_{s}, j_{\sigma(s)}}
$$

for subsets $I$ and $J$ of $\{1, \cdots, n\}$. We will use the following obvious relations :

$$
\begin{array}{rr}
X(I)=\sum_{v \in I-\{u\}} \delta_{i_{u}, i_{v}} X(I-\{u, v\}) & (u \in I \text { fixed }) \\
Y(J)=\sum_{v \in J-\{u\}} \delta_{j_{u}, j_{v}} Y(J-\{u, v\}) & (u \in J \text { fixed }) \\
Z(I, J)=\sum_{v \in J} \delta_{i_{u}, j_{v}} Z(I-\{u\}, J-\{v\}) & (u \in I \text { fixed }) .
\end{array}
$$

With these notations, (A.10) writes :

$$
\prod_{i_{1}, \cdots, i_{n} ; j_{1}, \cdots, j_{n}}=\sum_{0 \leq k \leq n / 2} f_{n, k} \sum_{\substack{J \subset\{1 \cdots n\} \\|J|=2 k}} \sum_{\substack{I \subset\{1 \cdots n\} \\|I|=2 k}} X(I) Z(\mathbf{C} I, \mathbf{C} J) Y(J)
$$

The sum on the subsets $I \subset\{1, \cdots, n\}$ is decomposed according to the four possible cases $(\emptyset,\{1\},\{2\},\{1,2\})$ of intersection of $I$ with the subset $\{1,2\}$.

$$
\begin{aligned}
& \prod_{i_{1}, \cdots, i_{n} ; j_{1}, \cdots, j_{n}}=\sum_{0 \leq k \leq n / 2} f_{n, k} \sum_{\substack{J \subset\{1 \cdots n\} \\
|J|=2 k}} \sum_{\substack{I \subset\{3 \cdots n\} \\
|I|=2 k}} X(I) Z\left(\mathbf{C}^{\prime} I \cup\{1,2\}, \mathbf{C} J\right) \\
+ & \sum_{\substack{I \subset\{3 \cdots n\} \\
|I|=2 k-1}} X(I \cup\{1\}) Z\left(\mathbf{C}^{\prime} I \cup\{2\}, \mathbf{C} J\right) \\
+ & \sum_{\substack{I \subset\{3 \cdots n\} \\
|I|=2 k-1}} X(I \cup\{2\}) Z\left(\mathbf{C}^{\prime} I \cup\{1\}, \mathbf{C} J\right) \\
+ & \left.\sum_{\substack{I \subset\{3 \cdots n\} \\
|I|=2 k-2}} X(I \cup\{1,2\}) Z\left(\mathbf{C}^{\prime} I, \mathbf{C} J\right)\right\} Y(J) .
\end{aligned}
$$

In this formula, the summation variable $I$ is obtained from the one of (A.80) by possibly removing the elements 1 and 2. Furthermore $\mathbf{C}^{\prime} I$ is the complementary set in $\{3, \cdots, n\}$ of the subset $I$, while $\mathbf{C} J$ is as before the complementary set of 
$J$ in $\{1, \cdots, n\}$. The explicitation of the indices $i_{1}$ and $i_{2}$ is then effected by the following obvious formulae (written for subsets $I \subset\{3, \cdots, n\}$ and $J \subset\{1, \cdots, n\}$ ), which can be as well deduced from (A.80) and (A.82) :

$$
\begin{gathered}
X(I \cup\{1\})=\sum_{u \in I} \delta_{i_{1} i_{u}} X(I-\{u\}) \\
X(I \cup\{2\})=\sum_{u \in I} \delta_{i_{2} i_{u}} X(I-\{u\}) \\
X(I \cup\{1,2\})=\delta_{i_{1} i_{2}} X(I)+\sum_{\substack{u, v \in I \\
u \neq v}} \delta_{i_{1} i_{u}} \delta_{i_{2} i_{v}} X(I-\{u, v\}) \\
Z(I \cup\{1\}, J)=\sum_{v \in J} \delta_{i_{1} j_{v}} Z(I, J-\{v\}) \\
Z(I \cup\{2\}, J)=\sum_{v \in J} \delta_{i_{2} j_{v}} Z(I, J-\{v\}) \\
Z(I \cup\{1,2\}, J)=\sum_{\substack{u, v \in J \\
u \neq v}} \delta_{i_{1} j_{u}} \delta_{i_{2} j_{v}} Z(I, J-\{u, v\}) .
\end{gathered}
$$

One obtains :

$$
\begin{aligned}
& \prod_{i_{1}, \cdots, i_{n} ; j_{1}, \cdots, j_{n}}=\sum_{0 \leq k \leq n / 2} f_{n, k} \sum_{\substack{J \subset\{1 \cdots n\} \\
|J|=2 k}} \\
& \left\{\sum_{\substack{I \subset\{3, \cdots n\} \\
|I|=2 k}} \sum_{\substack{u, v \notin J \\
u \neq v}} \delta_{i_{1} j_{u}} \delta_{i_{2} j_{v}} X(I) Z\left(\mathbf{C}^{\prime} I, \mathbf{C} J-\{u, v\}\right)\right. \\
& +\sum_{\substack{I \in\{3 \cdots n\} \\
|I|=2 k-1}} \sum_{u \in I} \sum_{v \notin J} \delta_{i_{1} i_{u}} \delta_{i_{2} j_{v}} X(I-\{u\}) Z\left(\mathbf{C}^{\prime} I, \mathbf{C} J-\{v\}\right) \\
& +\sum_{\substack{I \in\{3 \ldots n\} \\
|I|=2 k-1}} \sum_{u \in I} \sum_{v \notin J} \delta_{i_{2} i_{u}} \delta_{i_{1} j_{v}} X(I-\{u\}) Z\left(\mathbf{C}^{\prime} I, \mathbf{C} J-\{v\}\right) \\
& +\sum_{\substack{I C\{3 \cdots \cdots\} \\
\mid \subset I=2 k-2}} \delta_{i_{1} i_{2}} X(I) Z\left(\mathbf{C}^{\prime} I, \mathbf{C} J\right) \\
& \left.+\sum_{\substack{I \in\{3 \cdots n\} \\
|I|=2 k-2}} \sum_{\substack{u, v \in I \\
u \neq v}} \delta_{i_{1} i_{u}} \delta_{i_{2} i_{v}} X(I-\{u, v\}) Z\left(\mathbf{C}^{\prime} I, \mathbf{C} J\right)\right\} Y(J) \text {. }
\end{aligned}
$$

It is now straightforward to contract the indices $i_{1}$ and $i_{2}$ : 


$$
\begin{aligned}
& \sum_{i_{1}, i_{2}=1}^{D} \delta_{i_{1} i_{2}} \prod_{i_{1}, \cdots, i_{n} ; j_{1}, \cdots, j_{n}}=\sum_{0 \leq k \leq n / 2} f_{n, k} \\
& \left\{\sum_{\substack{J \subset\{1 \cdots n\} \\
|J|=2 k}} \sum_{\substack{I \subset\{3 \cdots n\} \\
|I|=2 k}} \sum_{\substack{u, v \notin J \\
u \neq v}} \delta_{j_{u} j_{v}} X(I) Z\left(\mathbf{C}^{\prime} I, \mathbf{C} J-\{u, v\}\right) Y(J)\right. \\
& +2 \sum_{\substack{J \subset\{1 \cdots n\} \\
|J|=2 k}} \sum_{\substack{I \subset\{3 \cdots n\} \\
|I|=2 k-1}} \sum_{u \in I} \sum_{v \notin J} \delta_{i_{u} j_{v}} X(I-\{u\}) Z\left(\mathbf{C}^{\prime} I, \mathbf{C} J-\{v\}\right) Y(J) \\
& +D \sum_{\substack{J \subset\{1 \cdots n\} \\
|J|=2 k}} \sum_{\substack{I \subset\{3 \cdots n\} \\
|I|=2 k-2}} X(I) Z\left(\mathbf{C}^{\prime} I, \mathbf{C} J\right) Y(J) \\
& \left.+\sum_{\substack{J \subset\{1 \cdots n\} \\
|J|=2 k}} \sum_{\substack{I \subset\{3 \cdots n\} \\
|I|=2 k-2}} \sum_{\substack{u, v \in I \\
u \neq v}} \delta_{i_{u} i_{v}} X(I-\{u, v\}) Z\left(\mathbf{C}^{\prime} I, \mathbf{C} J\right) Y(J)\right\} .
\end{aligned}
$$

Next we rewrite the first, second and last term in braces into the same form as the third one. Let us transform a partial sum in the first term as follows :

$$
\begin{aligned}
& \sum_{\substack{J \subset\{1 \cdots n\} \\
|J|=2 k}} \sum_{\substack{u, v \notin J \\
u \neq v}} \delta_{j_{u} j_{v}} Z\left(\mathbf{C}^{\prime} I, \mathbf{C} J-\{u, v\}\right) Y(J) \\
= & \sum_{\substack{J \subset\{1 \cdots n\} \\
|J|=2 k+2}} \sum_{\substack{u, v \in J \\
u \neq v}} \delta_{j_{u} j_{v}} Z\left(\mathbf{C}^{\prime} I, \mathbf{C} J\right) Y(J-\{u, v\}) .
\end{aligned}
$$

The first sum is, at fixed $u$ and $v$, over the subsets $J$ which contain $u$ and $v$, and the second sum is over the subsets $J$ which do not contain $u$ or $v$. To each subset of the first kind correspond the subset of the second kind obtained by removing $u$ and $v$, and the original first kind subset is recovered by including back $u$ and $v$. This proves (A.88). The sum over $u$ and $v$ in the right-hand side of (A.88) is then calculated. By summing (A.81) over $u \in J$, we have :

$$
\sum_{\substack{u, v \in J \\ u \neq v}} \delta_{j_{u} j_{v}} Y(J-\{u, v\})=|J| Y(J) .
$$

Combining (A.89) (with $|J|=2 k+2$ ) and (A.88), we have :

$$
\begin{gathered}
\sum_{\substack{J \subset\{1 \cdots n\} \\
|J|=2 k}} \sum_{\substack{u, v \notin J \\
u \neq v}} \delta_{j_{u} j_{v}} Z\left(\mathbf{C}^{\prime} I, \mathbf{C} J-\{u, v\}\right) Y(J) \\
=2(k+1) \sum_{\substack{J^{\prime} \subset\{1 \cdots n\} \\
\left|J^{\prime}\right|=2 k+2}} Z\left(\mathbf{C}^{\prime} I, \mathbf{C} J^{\prime}\right) Y\left(J^{\prime}\right)
\end{gathered}
$$


Let us transform a partial sum in the second term as follows :

$$
\begin{aligned}
& \sum_{\substack{I \subset\{3 \cdots n\} \\
|I|=2 k-1}} \sum_{u \in I} \sum_{v \notin J} \delta_{i_{u} j_{v}} X(I-\{u\}) Z\left(\mathbf{C}^{\prime} I, \mathbf{C} J-\{v\}\right) \\
= & \sum_{\substack{I \subset\{3 \cdots n\} \\
|\subset|=2 k-2}} \sum_{u \in \mathbf{C}^{\prime} I} \sum_{v \notin J} \delta_{i_{u} j_{v}} X(I) Z\left(\mathbf{C}^{\prime} I-\{u\}, \mathbf{C} J-\{v\}\right) .
\end{aligned}
$$

The first sum is, at fixed $u \in\{3, \cdots, n\}$, over the subsets $I$ which contain $u$, and the second sum is over the subsets $I$ which do not contain $u$. Formula (A.91) results from the fact that the two kinds of subsets are put in bijective correspondence by removing or including $u$. The sum over $u$ and $v$ in the right-hand side of (A.91) is then calculated. By summing (A.82) over $u \in I$, we have :

$$
\sum_{u \in I} \sum_{v \in J} \delta_{i_{u} j_{v}} Z(I-\{u\}, J-\{v\})=|I| Z(I, J) .
$$

Combining (A.92) (with $I \rightarrow \mathbf{C}^{\prime} I, J \rightarrow \mathbf{C} J,|I| \rightarrow n-2 k$ ) and (A.91), we have :

$$
\begin{gathered}
\sum_{\substack{I \subset\{3 \cdots n\} \\
|I|=2 k-1}} \sum_{u \in I} \sum_{v \notin J} \delta_{i_{u} j_{v}} X(I-\{u\}) Z\left(\mathbf{C}^{\prime} I, \mathbf{C} J-\{v\}\right) \\
=(n-2 k) \sum_{\substack{I \subset\{3 \cdots n\} \\
|I|=2 k-2}} X(I) Z\left(\mathbf{C}^{\prime} I, \mathbf{C} J\right) .
\end{gathered}
$$

For the last term, the sum over $u$ and $v$ is directly calculated by summing (A.80) over $u \in I$, we have :

$$
\sum_{\substack{u, v \in I \\ u \neq v}} \delta_{i_{u} i_{v}} X(I-\{u, v\})=|I| X(I)
$$

Using (A.90), (A.93), (A.94), the three last terms in (A.87) combine, with a coefficient

$$
2(n-2 k)+D+2(k-1)=2(n-k+D / 2-1)
$$

and formula (A.87) becomes:

$$
\sum_{i_{1}, i_{2}=1}^{D} \delta_{i_{1} i_{2}} \prod_{i_{1}, \cdots, i_{n} ; j_{1}, \cdots, j_{n}}=
$$




$$
\begin{gathered}
\sum_{0 \leq k \leq n / 2-1} f_{n, k} 2(k+1) \sum_{\substack{I \subset\{3 \cdots n\} \\
|I|=2 k}} \sum_{\substack{J \subset\{1 \cdots n\} \\
|J|=2 k+2}} X(I) Z\left(\mathbf{C}^{\prime} I, \mathbf{C} J\right) Y(J) \\
+\sum_{1 \leq k \leq n / 2} f_{n, k} 2(n-k+D / 2-1) \sum_{\substack{I \subset\{3 \cdots n\} \\
|I|=2 k-2}} \sum_{J \subset\{1 \cdots n\}} X(I) Z\left(\mathbf{C}^{\prime} I, \mathbf{C} J Y(J) .=2 k\right. \\
|\leq|
\end{gathered}
$$

We have suppressed zero terms in the $k$ sums : in the first sum, the existence of $J \subset\{1, \cdots, n\}$ with $|J|=2 k+2$ needs $2 k \leq n-2$, and in the second sum, the existence of I with $|I|=2 k-2$ needs $2 k \geq 2$. This adjustment of the summation bounds is important because now, after the change of variable $k \rightarrow k-1$, the first sum combines exactly with the second one :

$$
\begin{gathered}
\sum_{i_{1}, i_{2}=1}^{D} \delta_{i_{1} i_{2}} \prod_{i_{1}, \cdots, i_{n} ; j_{1}, \cdots, j_{n}}= \\
2 \sum_{1 \leq k \leq n / 2}\left[k f_{n, k-1}+(n-k+D / 2-1) f_{n, k}\right] \sum_{\substack{I \subset\{3 \cdots n\} \\
|I|=2 k-2}} \sum_{\substack{J \subset\{1 \cdots n\} \\
|J|=2 k}} X(I) Z\left(\mathbf{C}^{\prime} I, \mathbf{C} J\right) Y(J) .
\end{gathered}
$$

We find thus that, with the trial formula (A.10), the tracelessness condition 2) takes the form of the following recurrence relation for the coefficients $f_{n, k}$ :

$$
k f_{n, k-1}+(n-k+D / 2-1) f_{n, k}=0 \quad(1 \leq k \leq n / 2)
$$

and this recurrence relation, with the initial condition (A.78), uniquely determines the $f_{n, k}$ :

$$
f_{n, k}=(-1)^{k} \frac{k ! \Gamma(n-k+D / 2-1)}{n ! \Gamma(n+D / 2-1)}=(-1)^{k} \frac{1}{n !}\left(\begin{array}{c}
n+D / 2-1 \\
k
\end{array}\right)^{-1}
$$

where the second expression is defined for all required values $D \geq 1, n \geq 0,0 \leq k \leq$ $n / 2$, while the more explicit first one is ambiguous $(\infty / \infty)$ for $D=2, n=0, k=0$.

This result can be expressed with factorials, directly in case of $D$ even and by use of the duplication formula of the $\Gamma$ function in case of $D$ odd :

$$
\begin{gathered}
f_{n, k}=(-1)^{k} \frac{k !(n-k+D / 2-2) !}{n !(n+D / 2-2) !} \quad(D \text { even }) \\
f_{n, k}=(-1)^{k} 2^{-2 k} \frac{k !(n+D / 2-3 / 2) !(2 n-2 k+D-3) !}{n !(n-k+D / 2-3 / 2) !(2 n+D-3) !} \quad(D \text { odd }) .
\end{gathered}
$$


For the spatial dimension $D=3$, the expression (A.11) of $f_{n, k}$ is recovered.

\section{Calculation of the particular matrix elements from the expression of the projector}

By using the explicit expression (A.10) of the projector, it is of course possible to compute any matrix element needed. As a simple example, we present here the calculation of the particular matrix elements (A.3).

We may do all the calculations for a particle at rest $(v=(1, \overrightarrow{0}))$, and write the final result covariantly in term of the 4 -vectors $v, v_{i}, v_{f}$. Then we have :

$$
\begin{gathered}
v_{f}^{\mu_{1}} \cdots v_{f}^{\mu_{n}} \prod_{\mu_{1} \cdots \mu_{n} ; \nu_{1} \cdots \nu_{n}} v_{i}^{\nu_{1}} \cdots v_{i}^{\nu_{n}} \\
=\sum_{i_{1}, \cdots i_{n}, j_{1}, \cdots j_{n}} v_{f}^{i_{1}} \cdots v_{f}^{i_{n}} \prod_{i_{1}, \cdots, i_{n} ; j_{1}, \cdots, j_{n}} v_{i}^{j_{1}} \cdots v_{i}^{j_{n}} .
\end{gathered}
$$

The calculation of this from (A.10) simply amounts to the substitutions :

$$
\delta_{i_{r} i_{r^{\prime}}} \rightarrow\left(\vec{v}_{f}\right)^{2}, \quad \delta_{i_{s} j_{\sigma(x)}} \rightarrow\left(\vec{v}_{f} \cdot \vec{v}_{i}\right), \delta_{j_{t} j_{t^{\prime}}} \rightarrow\left(\vec{v}_{i}\right)^{2}
$$

and we have :

$$
\begin{gathered}
\sum_{i_{1}, \cdots i_{n}, j_{1}, \cdots j_{n}} v_{f}^{i_{1}} \cdots v_{f}^{i_{n}} \prod_{i_{1}, \cdots, i_{n} ; j_{1}, \cdots, j_{n}} v_{i}^{j_{1}} \cdots v_{i}^{j_{n}} \\
=\sum_{0 \leq k \leq n / 2} f_{n, k} \sum_{\substack{I, J \subset\{1 \cdots n\} \\
|I|=|J|=2 k}} \sum_{\mathcal{J} \in \mathcal{P}_{2}(I)} \sum_{\sigma \in \mathcal{B}(\mathbf{C} I, \mathbf{C} J)} \sum_{\mathcal{J}^{\prime} \in \mathcal{P}_{2}(J)}\left(\vec{v}_{f}\right)^{2 k}\left(\vec{v}_{f} \cdot \vec{v}_{i}\right)^{n-2 k}\left(\vec{v}_{i}\right)^{2 k} .
\end{gathered}
$$

The summand is independent of the summations variables $I, J, \mathcal{J}, \sigma, \mathcal{J}^{\prime}$, so that we have just to count the number of values they take. As well known, the number of subsets of cardinality $q$ in a set of cardinality $n$ is given by the binomial coefficient $\left(\begin{array}{l}n \\ q\end{array}\right)=\frac{n !}{q !(n-q) !}$, and the number $|\mathcal{B}(X, Y)|$ of bijections of a set $X$ on a set $Y$ is $|X|$ ! if $|X|=|Y|$ (and 0 else). The number $\left|\mathcal{P}_{2}(X)\right|$ of partitions of a set $X$ by two-

element subsets is $\frac{(2 k) !}{2^{k} k !}$ when $|X|=2 k$. This last number is easily obtained by first considering the set of decompositions of $X$ into ordered $k$-uples of ordered pairs. The number of such $k$-uples is the same $(2 k)$ ! as the number of permutations of $X$, and when the orderings (of the $k$-uples and of the pairs) are disregarded, there are $2^{k} k$ ! $k$-uples giving each partition by two-element subsets. Therefore the numbers of values taken by the summation variables in (A.104) are : 


$$
\begin{array}{ll}
\frac{n !}{(2 k) !(n-2 k) !} & \text { for } I \text { and for } J \\
\frac{(2 k) !}{2^{k} k !} & \text { for } \mathcal{J} \text { and for } \mathcal{J}^{\prime} \\
(n-2 k) ! & \text { for } \sigma .
\end{array}
$$

and from (A.104) we have :

$$
\begin{gathered}
\sum_{i_{1}, \cdots i_{n}, j_{1}, \cdots j_{n}} v_{f}^{i_{1}} \cdots v_{f}^{i_{n}} \prod_{i_{1}, \cdots, i_{n} ; j_{1}, \cdots, j_{n}} v_{i}^{j_{1}} \cdots v_{i}^{j_{n}} \\
=\sum_{0 \leq k \leq n / 2} f_{n, k}\left(\frac{n !}{(2 k) !(n-2 k) !}\right)^{2}\left(\frac{(2 k) !}{2^{k} k !}\right)^{2}(n-2 k) !\left(\vec{v}_{f}\right)^{2 k}\left(\vec{v}_{f} \cdot \vec{v}_{i}\right)^{n-2 k}\left(\vec{v}_{i}\right)^{2 k} \\
=\sum_{0 \leq k \leq n / 2} 2^{-2 k} \frac{(n !)^{2}}{(k !)^{2}(n-2 k) !} f_{n, k}\left(\vec{v}_{f}\right)^{2 k}\left(\vec{v}_{f} \cdot \vec{v}_{i}\right)^{n-2 k}\left(\vec{v}_{i}\right)^{2 k} .
\end{gathered}
$$

With $\left(\vec{v}_{f}\right)^{2},\left(\vec{v}_{f} \cdot \vec{v}_{i}\right),\left(\vec{v}_{i}\right)^{2}$ written in covariant form using (A.41), we recover (A.5) with $C_{n, k}$ given by

$$
C_{n, k}=(-1)^{k} 2^{-2 k} \frac{n !}{(k !)^{2}(n-2 k) !}\left(\begin{array}{c}
n+D / 2-2 \\
k
\end{array}\right)^{-1}
$$

for an arbitrary spatial dimension D. A compact expression like (A.3) is obtained from (A.5) and (A.106) by introducing the Gegenbauer polynomials $C_{n}^{\lambda}$, which can be defined by the generating functions :

$$
\sum_{n \geq 0} C_{n}^{\lambda}(x) t^{n}=\frac{1}{\left(1-2 x t+t^{2}\right)^{\lambda}}
$$

and have the following expressions :

$$
\begin{gathered}
C_{n}^{\lambda}(x)=\sum_{k}(-1)^{k}\left(\begin{array}{c}
n-k \\
k
\end{array}\right)\left(\begin{array}{c}
n-k+\lambda-1 \\
n-k
\end{array}\right)(2 x)^{n-2 k} \\
C_{n}^{\lambda}(x)=\sum_{k}(-1)^{k}\left(\begin{array}{c}
k+\lambda-1 \\
k
\end{array}\right)\left(\begin{array}{c}
n+2 \lambda-1 \\
n-2 k
\end{array}\right) x^{n-2 k}\left(1-x^{2}\right)^{k} .
\end{gathered}
$$

Indeed, (A.106) writes : 


$$
C_{n, k}=(-1)^{k} 2^{-2 k}\left(\begin{array}{c}
n+D / 2-2 \\
n
\end{array}\right)^{-1}\left(\begin{array}{c}
n-k \\
k
\end{array}\right)\left(\begin{array}{c}
n-k+D / 2-2 \\
n-k
\end{array}\right)
$$

and using (A.108) we have

$$
\sum_{0 \leq k \leq n / 2} C_{n, k} x^{n-2 k}=\frac{1}{2^{n}}\left(\begin{array}{c}
n+D / 2-2 \\
n
\end{array}\right)^{-1} C_{n}^{D / 2-1}(x)
$$

obtaining :

$$
\begin{gathered}
\sum_{i_{1}, \cdots i_{n}, j_{1}, \cdots j_{n}} v_{f}^{i_{1}} \cdots v_{f}^{i_{n}} \prod_{i_{1}, \cdots, i_{n} ; j_{1}, \cdots, j_{n}} v_{i}^{j_{1}} \cdots v_{i}^{j_{n}} \\
=\frac{1}{2^{n}}\left(\begin{array}{c}
n+D / 2-2 \\
n
\end{array}\right)^{-1}\left|\vec{v}_{f}\right|^{n}\left|\vec{v}_{i}\right|^{n} C_{n}^{D / 2-1}\left(\frac{\vec{v}_{f} \cdot \vec{v}_{i}}{\left|\vec{v}_{f}\right|\left|\vec{v}_{i}\right|}\right) .
\end{gathered}
$$

When $D=3$, expression (A.3) is recovered from (A.111) using $C_{n}^{1 / 2}(x)=P_{n}(x)$ and $\left(\begin{array}{c}n-1 / 2 \\ n\end{array}\right)=2^{-2 n} \frac{(2 n) !}{(n !)^{2}}$. Using in (A.111) the expression (A.109) of $C_{n}^{\lambda}(x)$, one obtains expression (A.6) with $C_{n, k}^{\prime}$ given by :

$$
C_{n, k}^{\prime}=(-1)^{k} \frac{1}{2^{n}}\left(\begin{array}{c}
n+D / 2-2 \\
n
\end{array}\right)^{-1}\left(\begin{array}{c}
k+D / 2-2 \\
k
\end{array}\right)\left(\begin{array}{c}
n+D-3 \\
n-2 k
\end{array}\right) .
$$

Notice that the expression (A.106') is ambiguous for $D=2, n \geq 1$ (while (A.106) is defined for all required values $D \geq 1, n \geq 0,0 \leq k \leq n / 2)$, and the ambiguity propagates to (A.111) and (A.112). If we want the case $D=2$ to be included in our formulae, we may use, instead the Gegenbauer polynomials, the Jacobi polynomials simply related to them by :

$$
C_{n}^{\lambda}(x)=\frac{(2 \lambda)_{n}}{(\lambda+1 / 2)_{n}} P_{n}^{(\lambda-1 / 2, \lambda-1 / 2)}(x)
$$

which have the following expressions :

$$
\begin{gathered}
P_{n}^{(\alpha, \alpha)}(x)=2^{-n} \sum_{k}(-1)^{k}\left(\begin{array}{c}
n+\alpha \\
k
\end{array}\right)\left(\begin{array}{c}
2 n-2 k+2 \alpha \\
n-2 k
\end{array}\right) x^{n-2 k} \\
P_{n}^{(\alpha, \alpha)}(x)=\sum_{k}(-1)^{k} 2^{-2 k}\left(\begin{array}{c}
n-k \\
k
\end{array}\right)\left(\begin{array}{c}
n+\alpha \\
n-k
\end{array}\right) x^{n-2 k}\left(1-x^{2}\right)^{k} .
\end{gathered}
$$


Rewriting (A.106) as :

$$
C_{n, k}=(-1)^{k}\left(\begin{array}{c}
2 n+D-3 \\
n
\end{array}\right)^{-1}\left(\begin{array}{c}
n+D / 2-3 / 2 \\
k
\end{array}\right)\left(\begin{array}{c}
2 n-2 k+D-3 \\
n-2 k
\end{array}\right)
$$

from (A.114) we see that :

$$
\begin{gathered}
\sum_{i_{1}, \cdots i_{n}, j_{1}, \cdots j_{n}} v_{f}^{i_{1}} \cdots v_{f}^{i_{n}} \prod_{i_{1}, \cdots, i_{n} ; j_{1}, \cdots, j_{n}} v_{i}^{j_{1}} \cdots v_{i}^{j_{n}} \\
=2^{n}\left(\begin{array}{c}
2 n+D-3 \\
n
\end{array}\right)^{-1}\left|\vec{v}_{f}\right|^{n}\left|\vec{v}_{i}\right|^{n} P_{n}^{(D-3) / 2,(D-3) / 2)}\left(\frac{\vec{v}_{f} \cdot \vec{v}_{i}}{\left|\vec{v}_{f}\right|\left|\vec{v}_{i}\right|}\right) .
\end{gathered}
$$

and using (A.115) we obtain :

$$
C_{n, k}^{\prime}=(-1)^{k} 2^{n-2 k}\left(\begin{array}{c}
2 n+D-3 \\
n
\end{array}\right)^{-1}\left(\begin{array}{c}
n-k \\
k
\end{array}\right)\left(\begin{array}{c}
n+D / 2-3 / 2 \\
n-k
\end{array}\right)
$$

The expression $\left(\mathrm{A} .106^{\prime \prime}\right)$ is ambiguous only for $D=1, n=1$, and so are (A.111') and $\left(\mathrm{A} .112^{\prime}\right)$.

Finally, let us consider the case $D=1$. It is in fact trivial. All $n$-rank tensor spaces $(n \geq 0)$ are of dimension 1 . All $n$-rank tensors are symmetric. The subspace of $n$-rank symmetric traceless tensors is the whole space when $n=0$ or 1 , and is the zero subspace when $n \geq 2$. Therefore, the projector on this subspace is the identity operator when $n=0$ or 1 (as for any dimension $D$ ), and is, when $n \geq 2$, the zero operator.

To see how this particular case is obtained with our results, we may apply eq. (A.111), which is well defined for $D=1$. First, due to $\vec{v}_{f} \cdot \vec{v}_{i}=\left|\vec{v}_{f}\right|\left|\vec{v}_{i}\right| \operatorname{sign}\left(\vec{v}_{f} \cdot \vec{v}_{i}\right)$, (A.111) writes

$$
\sum_{i_{1}, \cdots i_{n}, j_{1}, \cdots, j_{n}} v_{f}^{i_{1}} \cdots v_{f}^{i_{n}} \prod_{i_{1}, \cdots, i_{n} ; j_{1}, \cdots, j_{n}} v_{i}^{j_{1}} \cdots v_{i}^{j_{n}}=\frac{1}{2^{n}}\left(\begin{array}{c}
n-3 / 2 \\
n
\end{array}\right)^{-1} C_{n}^{-1 / 2}(1)\left(\vec{v}_{f} \cdot \vec{v}_{i}\right)^{n}
$$

(where the sum has only one term). Next $C_{n}^{-1 / 2}(1)$ is given by eq. (A.109) :

$$
C_{n}^{-1 / 2}(1)=\left(\begin{array}{c}
n-2 \\
n
\end{array}\right)=(-1)^{n}\left(\begin{array}{l}
1 \\
n
\end{array}\right)=\delta_{n, 0}-\delta_{n, 1}
$$

Then we have 


$$
\left.\frac{1}{2^{n}}\left(\begin{array}{c}
n-3 / 2 \\
n
\end{array}\right)^{-1}\right|_{n=0}=1 \quad,\left.\quad \frac{1}{2^{n}}\left(\begin{array}{c}
n-3 / 2 \\
n
\end{array}\right)^{-1}\right|_{n=1}=-1
$$

so that (A.116) becomes :

$$
\sum_{i_{1}, \cdots i_{n}, j_{1}, \cdots, j_{n}} v_{f}^{i_{1}} \cdots v_{f}^{i_{n}} \prod_{i_{1}, \cdots, i_{n} ; j_{1}, \cdots, j_{n}} v_{i}^{j_{1}} \cdots v_{i}^{j_{n}}=\delta_{n, 0}+\left(\vec{v}_{f} \cdot \vec{v}_{i}\right) \delta_{n, 1}
$$

and, as expected, this vanishes for $n \geq 2$.

\section{Appendix B.}

In this Appendix we give a manifestly covariant derivation of Bjorken and Uraltsev SR using the states and currents considered by Uraltsev [6]. He considers the forth component of the vector current, and initial and final $B^{*}$ states, allowing for spin flip transitions, i.e., with our notation, he takes $\Gamma_{1}=\Gamma_{2}=\gamma^{0}$, the initial and final states $\mathcal{B}_{i}=B^{*\left(\lambda_{i}\right)}(1, \mathbf{0}), \mathcal{B}_{f}=B^{*\left(\lambda_{f}\right)}\left(v_{f}^{0}, \mathbf{v}_{f}\right)$, and performing an expansion for small velocities. In the covariant language adopted here, the case he considers is

$$
\begin{gathered}
\Gamma_{1}=\Gamma_{2}=\psi_{i} \\
\mathcal{B}_{i}=P_{i+\ddagger_{i}} \quad \mathcal{B}_{f}=P_{f+\phi_{f}} .
\end{gathered}
$$

We realize that this case does not present the symmetry of the simple choice of Sections 3 and 4 , since both currents, projected in the $v_{i}$ direction, appear in a non-symmetric way relative to the initial and final states, that have four-velocities $v_{i}$ and $v_{f}$. This aspect, plus the $B^{*}$ polarization, complicates the calculation in a considerable way, since then all states $2_{3 / 2}^{+}, 1_{3 / 2}^{+}, 0_{1 / 2}^{+}$and $1_{1 / 2}^{+}$contribute. We give now the covariant version of Uraltsev calculation.

After a good deal of algebra, the r.h.s. of the general SR (38) writes, for the choice (B.1) :

$$
R\left(w_{i}, w_{f}, w_{i f}\right)=\xi\left(w_{i f}\right)\left\{\left(\varepsilon_{i} \cdot \varepsilon_{f}\right)\left(w_{i}+w_{f}\right)-\left(\varepsilon_{i} \cdot v_{f}\right)\left(\varepsilon_{f} \cdot v^{\prime}\right)-\left(\varepsilon_{f} \cdot v_{i}\right)\left(\varepsilon_{i} \cdot v^{\prime}\right)\right.
$$




$$
\left.-\left(2 w_{i}+1\right)\left[\left(\varepsilon_{i} \cdot \varepsilon_{f}\right)\left(w_{i f}+1\right)-\left(\varepsilon_{i} \cdot v_{f}\right)\left(\varepsilon_{f} \cdot v_{i}\right)\right]\right\}
$$

while the contribution of the different intermediate states is given by :

$$
\begin{aligned}
& L\left(0_{1 / 2}^{-}\right)=0 \\
& L\left(1_{1 / 2}^{-}\right)=\left\{-\left(w_{i}+1\right)\left[\left(\varepsilon_{i} \cdot \varepsilon_{f}\right)\left(w_{i f}+w_{i}\right)-\left(\varepsilon_{f} \cdot v_{i}\right)\left(\varepsilon_{i} \cdot v_{f}\right)+\left(\varepsilon_{f} \cdot v_{i}\right)\left(\varepsilon_{i} \cdot v^{\prime}\right)\right]\right. \\
& \left.+\left(\varepsilon_{i} \cdot v^{\prime}\right)\left[\left(\varepsilon_{f} \cdot v_{i}\right)\left(2 w_{i}-w_{f}+1\right)+\left(\varepsilon_{f} \cdot v^{\prime}\right)\left(w_{i f}-1\right)\right]\right\} \sum_{n} \xi^{(n)}\left(w_{i}\right) \xi^{(n)}\left(w_{f}\right)
\end{aligned}
$$

$L\left(2_{3 / 2}^{+}\right)=\left\{\frac{1}{2}\left(w_{i f}-w_{f} w_{i}\right)\left(w_{i}+1\right)\left[\left(\varepsilon_{i} \cdot \varepsilon_{f}\right) w_{i f}-\left(\varepsilon_{f} \cdot v_{i}\right)\left(\varepsilon_{i} \cdot v_{f}\right)\right.\right.$

$\left.+\left(\varepsilon_{i} \cdot \varepsilon_{f}\right) w_{i}+\left(\varepsilon_{f} \cdot v_{i}\right)\left(\varepsilon_{i} \cdot v^{\prime}\right)\right]-\frac{1}{2}\left(w_{i f}-w_{f} w_{i}\right)\left(\varepsilon_{i} \cdot v^{\prime}\right)\left[\left(\varepsilon_{f} \cdot v_{i}\right) w_{i}+\left(\varepsilon_{f} \cdot v_{i}\right) w_{i}-\left(\varepsilon_{f} \cdot v^{\prime}\right)\right]$

$+\frac{1}{6}\left(-2-2 w_{i}-2 w_{f}-3 w_{i f}+4 w_{i} w_{f}\right)\left(\varepsilon_{i} \cdot v^{\prime}\right)\left[\left(\varepsilon_{f} \cdot v_{i}\right)\left(1-w_{f}\right)+\left(\varepsilon_{f} \cdot v^{\prime}\right) w_{i f}\right]$

$-\frac{1}{2}\left[\left(\varepsilon_{i} \cdot v_{f}\right)\left(w_{i}+1\right)-\left(\varepsilon_{i} \cdot v^{\prime}\right) w_{i f}\right]\left(\varepsilon_{f} \cdot v^{\prime}\right)$

$-\frac{1}{2} w_{i}\left[\left(\varepsilon_{i} \cdot v_{f}\right)\left(w_{i}+1\right)-\left(\varepsilon_{i} \cdot v^{\prime}\right) w_{i f}\right]\left[\left(\varepsilon_{f} \cdot v_{i}\right)\left(1-w_{f}\right)+\left(\varepsilon_{f} \cdot v^{\prime}\right) w_{i f}\right]$

$+\frac{1}{2} w_{f}\left(\varepsilon_{i} \cdot v^{\prime}\right)\left(\varepsilon_{f} \cdot v^{\prime}\right)+w_{i}\left[\left(\varepsilon_{i} \cdot v_{f}\right)\left(w_{i}+1\right)-\left(\varepsilon_{i} \cdot v^{\prime}\right)\left(v_{i} \cdot v_{f}\right)\right]\left(\varepsilon_{f} \cdot v_{i}\right)$

$\left.-w_{i} w_{f}\left(\varepsilon_{i} \cdot v^{\prime}\right)\left(\varepsilon_{f} \cdot v_{i}\right)\right\} 3 \sum_{n} \tau_{3 / 2}^{(n)}\left(w_{i}\right) \tau_{3 / 2}^{(n)}\left(w_{f}\right)$

$L\left(1_{3 / 2}^{+}\right)=\left\{-\frac{1}{6}\left(1+w_{i}\right)\left(1+w_{f}\right) \frac{1}{4} \operatorname{Tr}\left[\psi_{i} \xi_{i} \gamma^{\sigma} \psi^{\prime} \gamma_{5}\right] \frac{1}{4} \operatorname{Tr}\left[\psi_{i} \xi_{f} \gamma_{\sigma} \psi_{f} \gamma_{5}\right]\right.$

$+\frac{1}{6}\left(1+w_{i}\right)\left(1+w_{f}\right) \frac{1}{4} \operatorname{Tr}\left[\psi_{i} \xi_{i} \psi^{\prime} \gamma^{\sigma} \gamma_{5}\right] \frac{1}{4} \operatorname{Tr}\left[\psi_{i} \xi_{f} \psi^{\prime} \gamma_{\sigma} \gamma_{5}\right]$

$\left.-\frac{1}{2}\left(1+w_{i}\right) \frac{1}{4} \operatorname{Tr}\left[\psi_{i} \xi_{i} \psi^{\prime} \psi_{f} \gamma_{5}\right] \frac{1}{4} \operatorname{Tr}\left[\psi_{i} \xi_{f} \psi^{\prime} \psi_{f} \gamma_{5}\right]\right\} 3 \sum_{n} \tau_{3 / 2}^{(n)}\left(w_{i}\right) \tau_{3 / 2}^{(n)}\left(w_{f}\right)$

$L\left(0_{1 / 2}^{+}\right)=\left(\varepsilon_{i} \cdot v^{\prime}\right)\left[\left(\varepsilon_{f} \cdot v_{i}\right)\left(1-w_{f}\right)+\left(\varepsilon_{f} \cdot v^{\prime}\right)\left(v_{i} \cdot v_{f}\right)\right] 4 \sum_{n} \tau_{1 / 2}^{(n)}\left(w_{i}\right) \tau_{1 / 2}^{(n)}\left(w_{f}\right)$

$L\left(1_{1 / 2}^{+}\right)=\left\{-\frac{1}{4} \operatorname{Tr}\left[\psi_{i} \notin_{i} \psi^{\prime} \gamma^{\sigma} \gamma_{5}\right] \frac{1}{4} \operatorname{Tr}\left[\psi_{i} \notin_{f} \psi_{f} \gamma_{\sigma} \gamma_{5}\right]\right.$

$\left.+\frac{1}{4} \operatorname{Tr}\left[\psi_{i} \notin_{i} \psi^{\prime} \gamma^{\sigma} \gamma_{5}\right] \frac{1}{4} \operatorname{Tr}\left[\psi_{i} \ddagger_{f} \psi^{\prime} \gamma_{\sigma} \gamma_{5}\right]\right\} 4 \sum_{n} \tau_{1 / 2}^{(n)}\left(w_{i}\right) \tau_{1 / 2}^{(n)}\left(w_{f}\right)$

$L\left(2_{3 / 2}^{-}\right)=\frac{1}{2}\left\{\left(w_{i f}-w_{i} w_{f}\right)\left(w_{i}+1\right) \frac{1}{4} \operatorname{Tr}\left[\psi_{i} \notin_{i} \psi^{\prime} \gamma^{\sigma} \gamma_{5}\right] \frac{1}{4} \operatorname{Tr}\left[\psi_{i} \xi_{f} \psi_{f} \gamma_{\sigma} \gamma_{5}\right]\right.$

$\left.-\left(w_{i f}-w_{i} w_{f}\right) \frac{1}{4} \operatorname{Tr}\left[\psi_{i} \notin_{i} \psi^{\prime} \gamma^{\sigma} \gamma_{5}\right] \frac{1}{4} \operatorname{Tr}\left[\psi_{i} \ddagger_{f} \psi^{\prime} \gamma_{\sigma} \gamma_{5}\right]\right\}$ 


$$
\begin{aligned}
& \left.+w_{i} \frac{1}{4} \operatorname{Tr}\left[\psi_{i} \ddagger_{i} \psi^{\prime} \psi_{f} \gamma_{5}\right] \frac{1}{4} \operatorname{Tr}\left[\psi_{i} \ddagger_{f} \psi^{\prime} \psi_{f} \gamma_{5}\right]\right\} 3 \sum_{n} \sigma_{3 / 2}^{(n)}\left(w_{i}\right) \sigma_{3 / 2}^{(n)}\left(w_{f}\right) \\
& L\left(1_{3 / 2}^{-}\right)=\left\{-\frac{1}{6}\left(w_{i}-1\right)\left(w_{f}-1\right)\left(w_{i}+1\right)\left[\left(\varepsilon_{i} \cdot \varepsilon_{f}\right)\left(w_{i f}+w_{i}\right)+\left(\varepsilon_{f} \cdot v_{i}\right)\left(\varepsilon_{i} \cdot v^{\prime}\right)-\left(\varepsilon_{f} \cdot v_{i}\right)\left(\varepsilon_{i} \cdot v_{f}\right)\right]\right. \\
& +\frac{1}{6}\left(w_{i}-1\right)\left(w_{f}-1\right)\left(\varepsilon_{i} \cdot v^{\prime}\right)\left[2\left(\varepsilon_{f} \cdot v_{i}\right) w_{i}-\left(\varepsilon_{f} \cdot v^{\prime}\right)\right] \\
& +\frac{1}{6}\left(1-9 w_{i f}+4 w_{i} w_{f}+2 w_{i}+2 w_{f}\right)\left(\varepsilon_{i} \cdot v^{\prime}\right)\left[\left(\varepsilon_{f} \cdot v_{i}\right)\left(1-w_{f}\right)+\left(\varepsilon_{f} \cdot v^{\prime}\right) w_{i f}\right] \\
& +\frac{1}{2}\left(w_{f}-1\right)\left(\varepsilon_{i} \cdot v^{\prime}\right)\left[2 w_{i}\left(\varepsilon_{f} \cdot v_{i}\right)-\left(\varepsilon_{f} \cdot v^{\prime}\right)\right] \\
& \left.+\frac{1}{2}\left(w_{i}-1\right)\left[\left(\varepsilon_{i} \cdot v_{f}\right)\left(w_{i}+1\right)-\left(\varepsilon_{i} \cdot v^{\prime}\right) w_{i f}\right]\left[\left(\varepsilon_{f} \cdot v^{\prime}\right) w_{i f}+\left(\varepsilon_{f} \cdot v_{i}\right)\left(1-w_{f}\right)\right]\right\} \\
& 3 \sum_{n} \sigma_{3 / 2}^{(n)}\left(w_{i}\right) \sigma_{3 / 2}^{(n)}\left(w_{f}\right)
\end{aligned}
$$

From

$$
\frac{1}{4} \operatorname{Tr}\left[\not \not \not \phi \not \not \gamma \gamma_{5}\right]=-i \varepsilon_{\mu \nu \rho \sigma} a^{\mu} b^{\nu} c^{\rho} d^{\sigma}
$$

one can express the contributions $L\left(1_{3 / 2}^{+}\right), L\left(1_{1 / 2}^{+}\right)$and $L\left(2_{3 / 2}^{-}\right)$in terms of scalar products. Indeeed, the product of two tensors $\varepsilon_{\mu \nu \rho \sigma}$ even non contracted can be expressed in terms of the tensor $g_{\mu \nu}$ :

$$
\begin{aligned}
& \varepsilon_{\mu \nu \rho \sigma} \varepsilon_{\mu^{\prime} \nu^{\prime} \rho^{\prime} \sigma^{\prime}}=-\operatorname{det}\left(g_{\alpha \alpha^{\prime}}\right) \quad\left(\alpha=\mu, \nu, \rho, \sigma ; \alpha^{\prime}=\mu^{\prime}, \nu^{\prime}, \rho^{\prime}, \sigma^{\prime}\right) \\
& g^{\mu \mu^{\prime}} \varepsilon_{\mu \nu \rho \sigma} \varepsilon_{\mu^{\prime} \nu^{\prime} \rho^{\prime} \sigma^{\prime}}=-\operatorname{det}\left(g_{\alpha \alpha^{\prime}}\right) \quad\left(\alpha=\nu, \rho, \sigma ; \alpha^{\prime}=\nu^{\prime}, \rho^{\prime}, \sigma^{\prime}\right) .
\end{aligned}
$$

From these relations one obtains, for the traces involved in $L\left(1_{3 / 2}^{+}\right), L\left(1_{1 / 2}^{+}\right)$and $L\left(2_{3 / 2}^{-}\right):$

$$
\begin{aligned}
& \frac{1}{4} \operatorname{Tr}\left[\psi_{i} \ddagger_{i} \psi^{\prime} \gamma^{\sigma} \gamma_{5}\right] \frac{1}{4} \operatorname{Tr}\left[\psi_{i} \ddagger_{f} \psi_{f} \gamma_{\sigma} \gamma_{5}\right] \\
& =-\left(\varepsilon_{i} \cdot v_{f}\right)\left(\varepsilon_{f} \cdot v^{\prime}\right)+w_{i}\left(\varepsilon_{i} \cdot v_{f}\right)\left(\varepsilon_{f} \cdot v_{i}\right)-\left(w_{i f} w_{i}-w_{f}\right)\left(\varepsilon_{i} \cdot \varepsilon_{f}\right) \\
& \frac{1}{4} \operatorname{Tr}\left[\psi_{i} \ddagger_{i} \psi^{\prime} \gamma^{\sigma} \gamma_{5}\right] \frac{1}{4} \operatorname{Tr}\left[\psi_{i} \xi_{f} \psi^{\prime} \gamma_{\sigma} \gamma_{5}\right] \\
& =-\left(\varepsilon_{i} \cdot v^{\prime}\right)\left(\varepsilon_{f} \cdot v^{\prime}\right)+w_{i}\left(\varepsilon_{i} \cdot v^{\prime}\right)\left(\varepsilon_{f} \cdot v_{i}\right)-\left(w_{i}^{2}-1\right)\left(\varepsilon_{i} \cdot \varepsilon_{f}\right) \\
& \frac{1}{4} \operatorname{Tr}\left[\psi_{i} \ddagger_{i} \psi^{\prime} \psi_{f} \gamma_{5}\right] \frac{1}{4} \operatorname{Tr}\left[\psi_{i} \ddagger_{f} \psi^{\prime} \psi_{f} \gamma_{5}\right] \\
& =\left(w_{i f}^{2}-1\right)\left(\varepsilon_{i} \cdot v^{\prime}\right)\left(\varepsilon_{f} \cdot v^{\prime}\right)-\left(w_{i f} w_{f}-w_{i}\right)\left(\varepsilon_{i} \cdot v^{\prime}\right)\left(\varepsilon_{f} \cdot v_{i}\right) \\
& -\left(w_{i f} w_{i}-w_{f}\right)\left(\varepsilon_{i} \cdot v_{f}\right)\left(\varepsilon_{f} \cdot v^{\prime}\right)+\left(w_{i f}-w_{i} w_{f}\right)\left(\varepsilon_{i} \cdot v_{f}\right)\left(\varepsilon_{f} \cdot v_{i}\right)
\end{aligned}
$$


$+\left(2 w_{i f} w_{i} w_{f}-w_{i f}^{2}-w_{i}^{2}-w_{f}^{2}+1\right)\left(\varepsilon_{i} \cdot \varepsilon_{f}\right)$.

From the latter expressions (B.12)-(B.14) and from (B.2)-(B.8) one gets finally for the equation (38) :

$$
\begin{aligned}
& \left\{-\left(w_{i}+1\right)\left(w_{i f}+w_{i}\right)\left(\varepsilon_{i} \cdot \varepsilon_{f}\right)+\left(w_{i}+1\right)\left(\varepsilon_{i} \cdot v_{f}\right)\left(\varepsilon_{f} \cdot v_{i}\right)+\left(w_{i}-w_{f}\right)\left(\varepsilon_{i} \cdot v^{\prime}\right)\left(\varepsilon_{f} \cdot v_{i}\right)\right. \\
& \left.+\left(w_{i f}-1\right)\left(\varepsilon_{i} \cdot v^{\prime}\right)\left(\varepsilon_{f} \cdot v^{\prime}\right)\right\} \sum_{n} \xi^{(n)}\left(w_{i}\right) \xi^{(n)}\left(w_{f}\right) \\
& +\left\{-\left(w_{i}+1\right)\left(4 w_{f} w_{i} w_{i f}+2 w_{f} w_{i}^{2}-w_{i}^{2}-w_{f}^{2}-2 w_{i f} w_{i}-3 w_{i f}^{2}+1\right)\left(\varepsilon_{i} \cdot \varepsilon_{f}\right)\right. \\
& +\left(w_{i}+1\right)\left(4 w_{i} w_{f}-3 w_{i f}+w_{i}\right)\left(\varepsilon_{i} \cdot v_{f}\right)\left(\varepsilon_{f} \cdot v_{i}\right)-\left(w_{i}+1\right)\left(w_{f}+1\right)\left(\varepsilon_{i} \cdot v_{f}\right)\left(\varepsilon_{f} \cdot v^{\prime}\right) \\
& +\left[\left(3 w_{i f}-2 w_{i} w_{f}+w_{f}\right)\left(w_{f}-w_{i}\right)-\left(w_{i}+1\right)^{2}\right]\left(\varepsilon_{i} \cdot v^{\prime}\right)\left(\varepsilon_{f} \cdot v_{i}\right) \\
& \left.+\left(2 w_{i} w_{f}-3 w_{i f}-w_{i}-w_{f}-1\right)\left(w_{i f}-1\right)\left(\varepsilon_{i} \cdot v^{\prime}\right)\left(\varepsilon_{f} \cdot v^{\prime}\right)\right\} \sum_{n} \tau_{3 / 2}^{(n)}\left(w_{i}\right) \tau_{3 / 2}^{(n)}\left(w_{f}\right) \\
& +4\left\{\left(w_{i f} w_{i}-w_{i}^{2}-w_{f}+1\right)\left(\varepsilon_{i} \cdot \varepsilon_{f}\right)-w_{i}\left(\varepsilon_{i} \cdot v_{f}\right)\left(\varepsilon_{f} \cdot v_{i}\right)+\left(\varepsilon_{i} \cdot v_{f}\right)\left(\varepsilon_{f} \cdot v^{\prime}\right)\right. \\
& \left.+\left(1+w_{i}-w_{f}\right)\left(\varepsilon_{i} \cdot v^{\prime}\right)\left(\varepsilon_{f} \cdot v_{i}\right)+\left(w_{i f}-1\right)\left(\varepsilon_{i} \cdot v^{\prime}\right)\left(\varepsilon_{f} \cdot v^{\prime}\right)\right\} \sum_{n} \tau_{1 / 2}^{(n)}\left(w_{i}\right) \tau_{1 / 2}^{(n)}\left(w_{f}\right) \\
& \left\{\left(w_{i}+2 w_{i} w_{f}-3 w_{f}^{2} w_{i}-w_{i}^{3}-2 w_{f} w_{i}^{3}-2 w_{i f}+2 w_{f} w_{i f}+2 w_{i}^{2} w_{i f}\right.\right. \\
& \left.+4 w_{f} w_{i}^{2} w_{i f}-3 w_{i} w_{i f}^{2}\right)\left(\varepsilon_{i} \cdot \varepsilon_{f}\right) \\
& +\left(-1+w_{f}+w_{i}^{2}-4 w_{f} w_{i}^{2}+3 w_{i} w_{i f}\right)\left(\varepsilon_{f} \cdot v_{i}\right)\left(\varepsilon_{i} \cdot v_{f}\right) \\
& +3\left(w_{i} w_{f}-w_{i f}\right)\left(\varepsilon_{i} \cdot v_{f}\right)\left(\varepsilon_{f} \cdot v^{\prime}\right) \\
& +\left(w_{f}-w_{f}^{2}-w_{i}+3 w_{i} w_{f}-2 w_{f}^{2} w_{i}+w_{i}^{2}+2 w_{f} w_{i}^{2}-3 w_{i f}\right. \\
& \left.+3 w_{f} w_{i f}-3 w_{i} w_{i f}\right)\left(\varepsilon_{f} \cdot v_{i}\right)\left(\varepsilon_{i} \cdot v^{\prime}\right) \\
& \left.+\left(w_{i f}-1\right)\left(-1+w_{i}+w_{f}+2 w_{i} w_{f}-3 w_{i f}\right)\left(\varepsilon_{i} \cdot v^{\prime}\right)\left(\varepsilon_{f} \cdot v^{\prime}\right)\right\} \sum_{n} \sigma_{3 / 2}^{(n)}\left(w_{i}\right) \sigma_{3 / 2}^{(n)}\left(w_{f}\right) \\
& =\xi\left(w_{i f}\right)\left\{\left[\left(w_{i}+w_{f}\right)-\left(2 w_{i}+1\right)\left(w_{i f}+1\right)\right]\left(\varepsilon_{i} \cdot \varepsilon_{f}\right)+\left(2 w_{i}+1\right)\left(\varepsilon_{i} \cdot v_{f}\right)\left(\varepsilon_{f} \cdot v_{i}\right)+\cdots\right. \\
& \left.-\left(\varepsilon_{i} \cdot v_{f}\right)\left(\varepsilon_{f} \cdot v^{\prime}\right)-\left(\varepsilon_{i} \cdot v^{\prime}\right)\left(\varepsilon_{f} \cdot v_{i}\right)\right\} .
\end{aligned}
$$

This expression is considerably more complicated than equation (48), that readily gives Uraltsev SR. We can choose the particular polarizations :

$$
\begin{array}{ll}
\varepsilon_{i}^{(1)}=\frac{v_{f}-w_{i f} v_{i}}{\sqrt{w_{i f}^{2}-1}} & \varepsilon_{f}^{(1)}=\frac{v_{i}-w_{i f} v_{f}}{\sqrt{w_{i f}^{2}-1}} \\
\varepsilon_{i}^{(2)}=\frac{v^{\prime}-w_{i} v_{i}}{\sqrt{w_{i}^{2}-1}} & \varepsilon_{f}^{(2)}=\frac{v^{\prime}-w_{f} v_{f}}{\sqrt{w_{f}^{2}-1}}
\end{array}
$$

that satisfy $\varepsilon_{i}^{2}=-1, \varepsilon_{i} \cdot v_{i}=0, \varepsilon_{f}^{2}=-1, \varepsilon_{f} \cdot v_{f}=0$. 
We can consider the following different cases :

$$
\begin{array}{ll}
(1) \quad \varepsilon_{i}=\varepsilon_{i}^{(1)} \quad, \quad \varepsilon_{f}=\varepsilon_{f}^{(1)} ; \\
(2) \quad \varepsilon_{i}=\varepsilon_{i}^{(2)} \quad, \quad \varepsilon_{f}=\varepsilon_{f}^{(1)} ; \\
(3) \quad \varepsilon_{i}=\varepsilon_{i}^{(1)}, \quad \varepsilon_{f}=\varepsilon_{f}^{(2)} ; \\
(4) \quad \varepsilon_{i}=\varepsilon_{i}^{(2)}, \quad, \quad \varepsilon_{f}=\varepsilon_{f}^{(2)} .
\end{array}
$$

These four different cases exhaust the number of independent SR in the case under consideration, characterized by (B.1).

That there are only four independent SR can be seen by the following argument. If, in the general SR (B.15), we make the replacements (the sum over $\alpha$ denotes the sum over the different polarizations) :

$$
\begin{aligned}
& \varepsilon_{i}^{\mu} \rightarrow \sum_{\alpha} \varepsilon_{i}^{(\alpha) \mu}=g^{\rho \mu}-v_{i}^{\rho} v_{i}^{\mu} \\
& \varepsilon_{f}^{\nu} \rightarrow \sum_{\alpha} \varepsilon_{f}^{(\alpha) \nu}=g^{\sigma \nu}-v_{f}^{\sigma} v_{i}^{\nu}
\end{aligned}
$$

one obtains a set of tensorial identities, that depend only on $v_{i}, v_{f}$ and $v^{\prime}$ :

$$
X^{\rho \sigma}\left(v_{i}, v_{f}, v^{\prime}\right)=0
$$

From these 16 identities one obtains 9 scalar identities saturating with all the pairs $v_{i \rho} v_{i \sigma}, v_{f \rho} v_{f \sigma}, v_{\rho}^{\prime} v_{\sigma}^{\prime}, v_{i \rho} v_{f \sigma}, \ldots$

$$
\begin{aligned}
& v_{i \rho} v_{i \sigma} X^{\rho \sigma}\left(v_{i} v_{f}, v^{\prime}\right)=0 \\
& \ldots
\end{aligned}
$$

plus 3 other scalar identities, identically vanishing,

$$
\begin{aligned}
& \varepsilon_{\mu \nu \rho \sigma} v_{i}^{\mu} v_{f}^{\nu} X^{\rho \sigma}\left(v_{i} v_{f}, v^{\prime}\right) \equiv 0 \\
& \ldots
\end{aligned}
$$

However, among these equations, only 4 are independent, corresponding to the two non-vanishing products

$$
v_{f \rho}\left(g^{\rho \mu}-v_{i}^{\rho} v_{i}^{\mu}\right)=v_{f}^{\mu}-w_{i f} v_{i}^{\mu}
$$




$$
v_{\rho}^{\prime}\left(g^{\rho \mu}-v_{i}^{\rho} v_{i}^{\mu}\right)=v^{\prime \mu}-w_{i} v_{i}^{\mu}
$$

that must be combined with the other two four-vectors :

$$
\begin{gathered}
v_{i \rho}\left(g^{\rho \mu}-v_{f}^{\rho} v_{f}^{\mu}\right)=v_{i}^{\mu}-w_{i f} v_{f}^{\mu} \\
v_{\rho}^{\prime}\left(g^{\rho \mu}-v_{f}^{\rho} v_{f}^{\mu}\right)=v^{\prime \mu}-w_{f} v_{f}^{\mu} .
\end{gathered}
$$

Let us now write this SR (B.15) for the different cases. We need the following scalar products :

$$
\begin{array}{cc}
\varepsilon_{i}^{(1)} \cdot v_{f}=-\frac{w_{i f}^{2}-1}{\sqrt{w_{i f}^{2}-1}} & \varepsilon_{i}^{(1)} \cdot v^{\prime}=-\frac{w_{i f} w_{i}-w_{f}}{\sqrt{w_{i f}^{2}-1}} \\
\varepsilon_{i}^{(2)} \cdot v_{f}=-\frac{w_{i f} w_{i}-w_{f}}{\sqrt{w_{i}^{2}-1}} & \varepsilon_{i}^{(2)} \cdot v^{\prime}=-\frac{w_{i}^{2}-1}{\sqrt{w_{i}^{2}-1}} \\
\varepsilon_{f}^{(1)} \cdot v_{i}=-\frac{w_{i f}^{2}-1}{\sqrt{w_{i f}^{2}-1}} & \varepsilon_{f}^{(1)} \cdot v^{\prime}=-\frac{w_{i f} w_{f}-w_{i}}{\sqrt{w_{i f}^{2}-1}} \\
\varepsilon_{f}^{(2)} \cdot v_{i}=-\frac{w_{i f} w_{f}-w_{i}}{\sqrt{w_{f}^{2}-1}} & \varepsilon_{f}^{(2)} \cdot v^{\prime}=-\frac{w_{f}^{2}-1}{\sqrt{w_{f}^{2}-1}} \\
\varepsilon_{i}^{(1)} \cdot \varepsilon_{f}^{(1)}=\frac{w_{i f}\left(w_{i f}^{2}-1\right)}{\sqrt{w_{i f}^{2}-1} \sqrt{w_{i f}^{2}-1}} & \varepsilon_{i}^{(2)} \cdot \varepsilon_{f}^{(1)}=\frac{w_{i f}\left(w_{i f} w_{i}-w_{f}\right)}{\sqrt{w_{i}^{2}-1} \sqrt{w_{i f}^{2}-1}} \\
\varepsilon_{i}^{(1)} \cdot \varepsilon_{f}^{(2)}=\frac{w_{i f}\left(w_{i f} w_{f}-w_{i}\right)}{\sqrt{w_{f}^{2}-1}} \sqrt{w_{i f}^{2}-1} & \varepsilon_{i}^{(2)} \cdot \varepsilon_{f}^{(2)}=\frac{w_{i f} w_{i} w_{f}-w_{i}^{2}-w_{f}^{2}+1}{\sqrt{w_{i f}^{2}-1} \sqrt{w_{i f}^{2}-1}}
\end{array}
$$

Since equation (B.15) is linear in $\varepsilon_{i}$ and in $\varepsilon_{f}$, in deducing the equation for the different cases we can multiply (B.15) by the denominators defining the polarizations in (B.16). We thus obtain from (B.15) four different equations for the different cases (B.17).

If, in particular, we make $w_{i}=w_{f}=w$, we obtain the following equations, for the different cases considered :

$$
\text { (1) } \varepsilon_{i}=\varepsilon_{i}^{(1)}, \varepsilon_{f}=\varepsilon_{f}^{(1)}:
$$




$$
\begin{aligned}
& -\left(w_{i f}-1\right)\left(1+w-w^{2}-w_{i f}+2 w w_{i f}+3 w^{2} w_{i f}+w w_{i f}^{2}\right) \sum_{n}\left[\xi^{(n)}(w)\right]^{2} \\
& +\left(w_{i f}-1\right)\left(w-2 w^{2}-4 w^{3}+2 w^{4}+2 w_{i f}+w w_{i f}-4 w^{2} w_{i f}-6 w^{4} w_{i f}+2 w_{i f}^{2}+3 w w_{i f}^{2}+6 w^{2} w_{i f}^{2}\right. \\
& \left.-4 w^{3} w_{i f}^{2}+3 w w_{i f}^{3}\right) \sum_{n}\left[\tau_{3 / 2}^{(n)}(w)\right]^{2} \\
& -4\left(w_{i f}-1\right)\left(w-w^{2}-w_{i f}+3 w^{2} w_{i f}-w_{i f}^{2}-w w_{i f}^{2}\right) \sum_{n}\left[\tau_{1 / 2}^{(n)}(w)\right]^{2} \\
& +\left(w_{i f}-1\right)\left(1-w-2 w^{2}+2 w^{4}+w_{i f}+3 w w_{i f}-4 w^{3} w_{i f}-6 w^{4} w_{i f}-3 w_{i f}^{2}\right. \\
& \left.+w\left(w_{i f}\right)^{2}+10 w^{2} w_{i f}^{2}+4 w^{3} w_{i f}^{2}-3 w_{i f}^{3}-3 w w_{i f}^{3}\right) \sum_{n}\left[\sigma_{3 / 2}^{(n)}(w)\right]^{2}+\cdots \\
& =-\left(w_{i f}-1\right)\left(w_{i f}+1\right)\left(1+w_{i f}+2 w w_{i f}\right) \xi\left(w_{i f}\right) \\
& \text { (2) } \varepsilon_{i}=\varepsilon_{i}^{(2)}, \varepsilon_{f}=\varepsilon_{f}^{(1)}: \\
& -w(w+1)\left(w_{i f}-1\right)\left(w+w_{i f}\right) \sum_{n}\left[\xi^{(n)}(w)\right]^{2} \\
& +(w+1)\left(w_{i f}-1\right)\left(1-2 w^{2}-2 w^{4}+w_{i f}+w w_{i f}+2 w^{2} w_{i f}-4 w^{3} w_{i f}+3 w w_{i f}^{2}\right) \sum_{n}\left[\tau_{3 / 2}^{(n)}(w)\right]^{2} \\
& +4(w-1)(w+1)\left(w_{i f}-1\right)\left(1-w+w_{i f}\right) \sum_{n}\left[\tau_{1 / 2}^{(n)}(w)\right]^{2} \\
& +(w-1)(w+1)\left(w_{i f}-1\right)\left(2 w^{2}-2 w^{3}-3 w_{i f}+2 w w_{i f}+4 w^{2} w_{i f}-3 w_{i f}^{2}\right) \sum_{n}\left[\sigma_{3 / 2}^{(n)}(w)\right]^{2} \\
& +\cdots=-(w+1)\left(w_{i f}+1\right)\left(w_{i f}-1\right)(2 w-1) \xi\left(w_{i f}\right) \\
& \text { (3) } \varepsilon_{i}=\varepsilon_{i}^{(1)}, \varepsilon_{f}=\varepsilon_{f}^{(2)}: \\
& -w(w+1)\left(w_{i f}-1\right)\left(w+w_{i f}\right) \sum_{n}\left[\xi^{(n)}(w)\right]^{2} \\
& +(w+1)\left(w_{i f}-1\right)\left(1-2 w^{2}-2 w^{4}+w_{i f}+w w_{i f}+2 w^{2} w_{i f}-4 w^{3} w_{i f}+3 w w_{i f}^{2}\right) \sum_{n}\left[\tau_{3 / 2}^{(n)}(w)\right]^{2} \\
& +4(w-1)(w+1)\left(w_{i f}-1\right)\left(1-w+w_{i f}\right) \sum_{n}\left[\tau_{1 / 2}^{(n)}(w)\right]^{2} \\
& +(w-1)\left(w_{i f}-1\right)\left(-w^{2}+3 w^{3}-2 w^{4}-3 w_{i f}-w w_{i f}+9 w^{2} w_{i f}+w^{3} w_{i f}-3 w_{i f}^{2}-3 w w_{i f}^{2}\right) \\
& \sum_{n}\left[\sigma_{3 / 2}^{(n)}(w)\right]^{2}+\cdots=-(w+1)\left(w_{i f}+1\right)\left(w_{i f}-1\right)(2 w-1) \xi\left(w_{i f}\right) \\
& \text { (4) } \varepsilon_{i}=\varepsilon_{i}^{(2)}, \varepsilon_{f}=\varepsilon_{f}^{(2)}: \\
& (w+1)^{2}\left(-1+w+w^{2}-w w_{i f}\right) \sum_{n}\left[\xi^{(n)}(w)\right]^{2} \\
& +(w+1)^{2}\left(-w-2 w^{2}+4 w^{3}+2 w^{4}+2 w_{i f}-2 w w_{i f}-2 w^{2} w_{i f}+4 w^{3} w_{i f}+3 w w_{i f}^{2}\right)
\end{aligned}
$$




$$
\begin{aligned}
& \sum_{n}\left[\tau_{3 / 2}^{(n)}(w)\right]^{2}+4(w-1)^{2}(w+1)\left(w+w_{i f}\right) \sum_{n}\left[\tau_{1 / 2}^{(n)}(w)\right]^{2} \\
& +(w-1)^{2}(w+1)\left(1-2 w^{2}+2 w^{3}-2 w w_{i f}+4 w^{2} w_{i f}-3 w_{i f}^{2}\right) \sum_{n}\left[\sigma_{3 / 2}^{(n)}(w)\right]^{2}+\cdots \\
& =(w+1)\left(-1+3 w-w_{i f}-3 w w_{i f}+2 w^{2} w_{i f}\right) \xi\left(w_{i f}\right) .
\end{aligned}
$$

Let us now obtain the SR that can be obtained without deriving the function $\xi\left(w_{i f}\right)$ :

$$
\text { (1) } \varepsilon_{i}=\varepsilon_{i}^{(1)}, \varepsilon_{f}=\varepsilon_{f}^{(1)}:
$$

Dividing by $\left(w_{i f}-1\right)$ and taking the limit $w_{i f} \rightarrow 1$, one gets :

$$
\begin{aligned}
& -2(w+1)^{2} \sum_{n}\left[\xi^{(n)}(w)\right]^{2}-4(w-1)(w+1)^{3} \sum_{n}\left[\tau_{3 / 2}^{(n)}(w)\right]^{2} \\
& -8(w-1)(w+1) \sum_{n}\left[\tau_{1 / 2}^{(n)}(w)\right]^{2}-4\left(w^{2}-1\right)^{2} \sum_{n}\left[\sigma_{3 / 2}^{(n)}(w)\right]^{2}+\cdots \\
& =-4(w+1) \\
& \quad(2) \varepsilon_{i}=\varepsilon_{i}^{(2)}, \varepsilon_{f}=\varepsilon_{f}^{(1)} \text { and }(3) \varepsilon_{i}=\varepsilon_{i}^{(1)}, \varepsilon_{f}=\varepsilon_{f}^{(2)}:
\end{aligned}
$$

Dividing by $\left(w_{i f}-1\right)$ and taking the limit $w_{i f} \rightarrow 1$, one obtains :

$$
\begin{aligned}
& -w(w+1)^{2} \sum_{n}\left[\xi^{(n)}(w)\right]^{2}-2(w-1)(w+1)^{4} \sum_{n}\left[\tau_{3 / 2}^{(n)}(w)\right]^{2} \\
& -4(w-1)(w+1)(w-2) \sum_{n}\left[\tau_{1 / 2}^{(n)}(w)\right]^{2}+2\left(w^{2}-1\right)^{2}(3-w) \sum_{n}\left[\sigma_{3 / 2}^{(n)}(w)\right]^{2}+\cdots \\
& =-2(w+1)(2 w-1) \\
& \quad \text { (4) } \varepsilon_{i}=\varepsilon_{i}^{(2)}, \varepsilon_{f}=\varepsilon_{f}^{(2)}:
\end{aligned}
$$

Taking the limit $w_{i f} \rightarrow 1$, one gets :

$$
\begin{aligned}
& (w-1)(w+1)^{3} \sum_{n}\left[\xi^{(n)}(w)\right]^{2}+2(w+1)^{4}(w-1)^{2} \sum_{n}\left[\tau_{3 / 2}^{(n)}(w)\right]^{2} \\
& +4(w-1)^{2}(w+1)^{2} \sum_{n}\left[\tau_{1 / 2}^{(n)}(w)\right]^{2}+2\left(w^{2}-1\right)^{3} \sum_{n}\left[\sigma_{3 / 2}^{(n)}(w)\right]^{2}+\cdots \\
& =2(w+1)^{2}(w-1)
\end{aligned}
$$


From (B.26)-(B.27) one gets two sum rules

$$
\begin{aligned}
& \frac{w+1}{2} \sum_{n}\left[\xi^{(n)}(w)\right]^{2}+(w-1)\left\{2 \sum_{n}\left[\tau_{1 / 2}^{(n)}(w)\right]^{2}+(w+1)^{2} \sum_{n}\left[\tau_{3 / 2}^{(n)}(w)\right]^{2}\right\} \\
& +(w+1)(w-1)^{2} \sum_{n}\left[\sigma_{3 / 2}^{(n)}(w)\right]^{2}+\cdots=1 \\
& w \frac{w+1}{2} \sum_{n}\left[\xi^{(n)}(w)\right]^{2}+(w-1)\left\{(w+1)^{3} \sum_{n}\left[\tau_{3 / 2}^{(n)}(w)\right]^{2}\right. \\
& \left.+2(w-2) \sum_{n}\left|\tau_{1 / 2}^{(n)}(w)\right|^{2}\right\}-(w+1)(w-1)^{2}(3-w) \sum_{n}\left[\sigma_{3 / 2}^{(n)}(w)\right]^{2}+\cdots=2 w-1(
\end{aligned}
$$

The first SR is Bjorken SR (53).

$$
\begin{aligned}
& \text { Eliminating } \frac{w+1}{2} \sum_{n}\left|\xi^{(n)}(w)\right|^{2} \text { between (B.28) and (B.29) one obtains : } \\
& (w+1)^{2} \sum_{n}\left|\tau_{3 / 2}^{(n)}(w)\right|^{2}-4 \sum_{n}\left|\tau_{1 / 2}^{(n)}(w)\right|^{2}-3\left(w^{2}-1\right) \sum_{n}\left[\sigma_{3 / 2}^{(n)}(w)\right]^{2}+\cdots=1
\end{aligned}
$$

Equation (B.34) is another generalization of Uraltsev SR for $w \neq 1$; it reduces indeed to $(57)$ for $w=1$. Notice that the states $\frac{3}{2}^{-}$contribute at order $(w-1)$ to (B.34), while they do not contribute at all to the generalization of Uraltsev SR for $w \neq 1$ (67). There is no contradiction : these are two different generalizations, and the difference can be traced back to the fact that the former is obtained from asymmetric currents $\left\{\psi_{i}, \psi_{i}\right\}$ while the latter is obtained from symmetric currents $\left\{\psi_{i} \gamma_{5}, \psi_{f} \gamma_{5}\right\}$ relative to the initial and final four-velocities.

\section{Acknowledgements}

We are indebted to Zoltan Ligeti for encouragements and for pointing out to us the interest of publishing the derivation of Uraltsev sum rule given in Section 3, and to Nikolai Uraltsev for long standing discussions on QCD in the heavy quark limit. We acknowledge support from the EC contract HPRN-CT-2002-00311 (EURIDICE).

\section{References}

[1] J. D. Bjorken, invited talk at Les Rencontres de la Vallée d'Aoste, La Thuile, SLAC-PUB-5278, 1990 ; N. Isgur and M. B. Wise, Phys. Rev. D43, 819 (1991). 
[2] I. Bigi, M. Shifman, N. G. Uraltsev and A. Vainshtein, Phys. Rev. D52, 196 (1995).

[3] I. I. Bigi, M. A. Shifman and N. Uraltsev, Ann. Rev. Nucl. Part. Sci. 47, 591 (1997), hep-ph/9703290.

[4] A. K. Leibovich, Z. Ligeti, I. W. Steward and M. Wise, Phys. Rev. D57, 308 (1998).

[5] A. Le Yaouanc, D. Melikhov, V. Morénas, L. Oliver, O. Pène and J.-C. Raynal, Phys. Lett. B480, 119 (2000).

[6] N. Uraltsev, Phys. Lett. B501, 86 (2001).

[7] N. Uraltsev, J. Phys. G 27, 1081 (2001), hep-ph/0012336.

[8] A. Le Yaouanc et al., to appear.

[9] K. G. Wilson, Phys. Rev. 179, 1499 (1969) ; Phys. Rep. 12, 75 (1974).

[10] B. Blok, K. Koyrakh, M. Shifman and A. L. Vainshtein, Phys. Rev. D49, 3356 (1994).

[11] B. Bakamjian and L. H. Thomas, Phys. Rev. 92, 1300 (1953).

[12] A. Le Yaouanc, L. Oliver, O. Pène and J.-C. Raynal, Phys. Lett. B365, 319 (1996).

[13] A. Le Yaouanc, L. Oliver, O. Pène, J.-C. Raynal and V. Morénas, Phys. Lett. B520, 25 (2001).

[14] A. F. Falk et al., Nucl. Phys. B343, 1 (1990) ; J. D. Bjorken, Proceedings of the 18th SLAC Summer Institute on Particle Physics, pp. 167, Stanford, July 1990, ed. by J.F. Hawthorne (SLAC, Stanford, 1991).

[15] A. Le Yaouanc, L . Oliver and J.-C. Raynal, LPT Orsay 02-94, hep-ph/0210231.

[16] A. Falk, Nucl. Phys. B378, 79 (1992). 
[17] A. Le Yaouanc, L. Oliver, O. Pène and J.-C. Raynal, Phys. Lett. B386, 304 (1996) ; A. Le Yaouanc, L. Oliver, O. Pène, J.-C. Raynal and V. Morénas, Phys. Lett. 520, 25 (2001).

[18] V. Morénas, A. Le Yaouanc, L. Oliver, O. Pène and J.-C. Raynal, Phys. Rev. D56, 5668 (1997).

[19] We have recently demonstrated rigorously this bound using a different class of SR based on the formalism of the present paper, cf. hep-ph/0210231 [15]. 